Aus dem Institut für Allgemeinmedizin

(Prof. Dr. med. Eva Hummers-Pradier)

im Zentrum Innere Medizin

der Medizinischen Fakultät der Universität Göttingen

\title{
NiederLASSUNG IN LÄNDLICHEN Gebieten NiedERSACHSENS AUS HAUSÄRZTLICHER SICHT
}

\author{
INAUGURAL - DISSERTATION \\ zur Erlangung des Doktorgrades \\ der Medizinischen Fakultät der \\ Georg-August-Universität zu Göttingen
}

vorgelegt von

Annika Heubrock

aus

Münster

Göttingen 2014 
Dekan:

\section{Berichterstatter:}

II. Berichterstatter/in:

III. Berichterstatter/in:

Tag der mündlichen Prüfung:
Prof. Dr. rer. nat. H. K. Kroemer

Prof. Dr. disc. pol. W. Himmel

Prof. Dr. med. A. Klockgether-Radke

Prof. Dr. hum. biol. M. Schön 
Inhalt

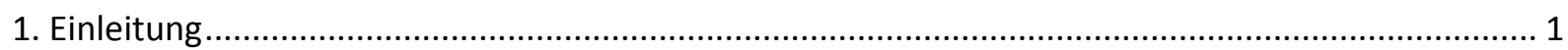

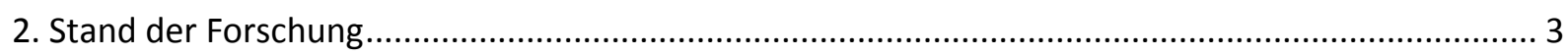

2.1 Entwicklungen der Hausarztzahlen mit Schwerpunkt Niedersachsen ........................................ 3

2.2 Einflussfaktoren auf die Berufs- und Niederlassungsentscheidung ......................................... 6

2.3 Internationale Bemühungen zur Erhöhung der Hausarztzahlen ................................................ 7

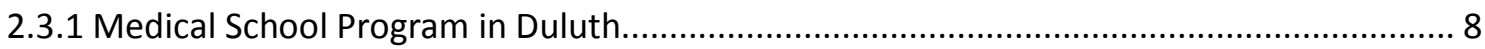

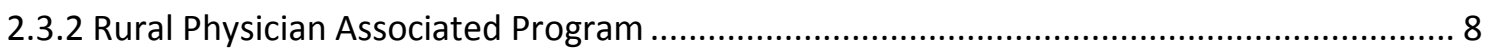

2.3.3 Ergebnisse des Medical School Program in Duluth und des RPAP ................................... 9

2.4 Bemühungen zur Erhöhung der Hausarztzahlen in Deutschland................................................ 9

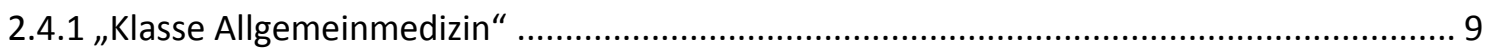

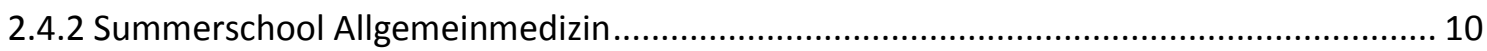

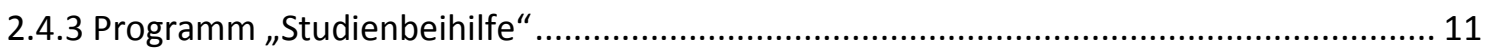

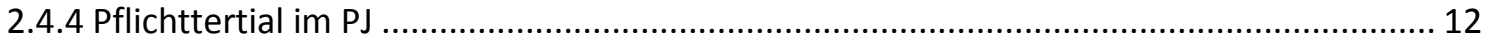

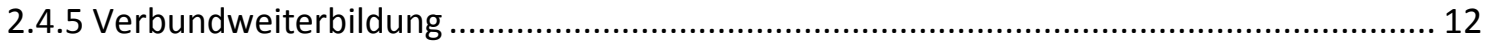

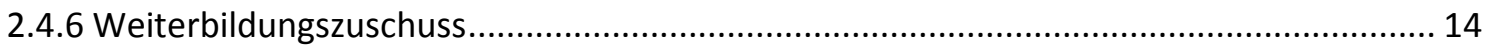

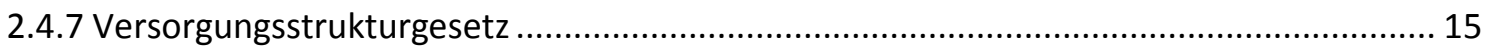

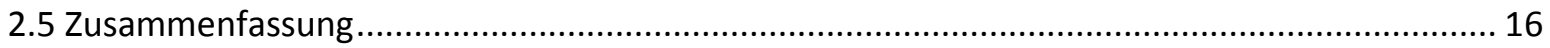

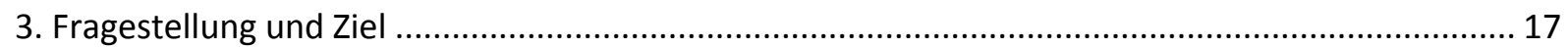

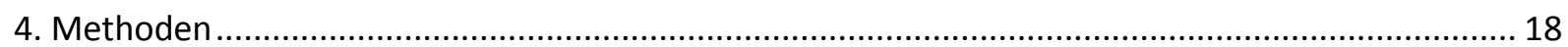

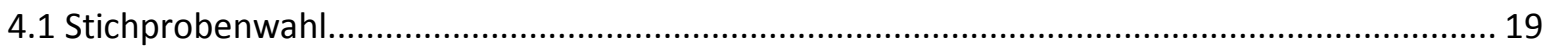

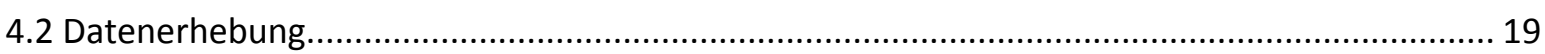

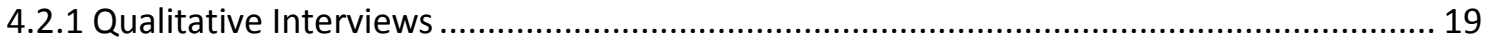

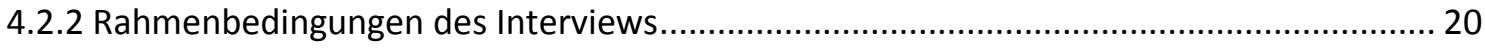

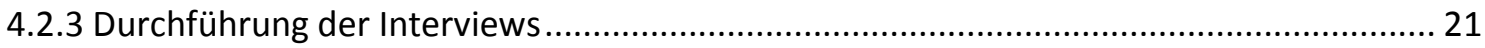

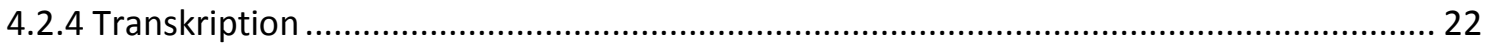

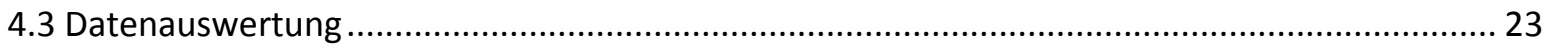

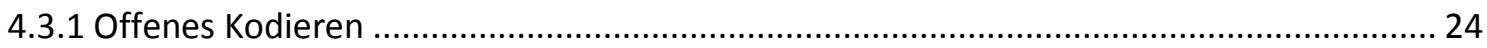

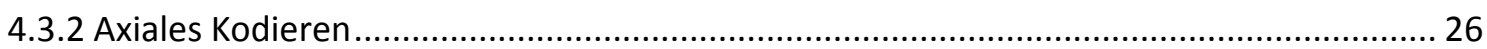

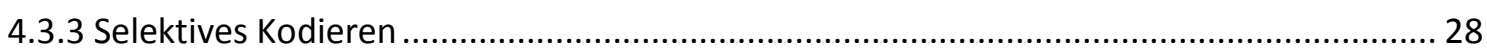

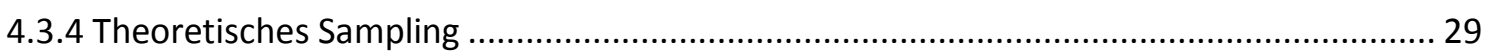

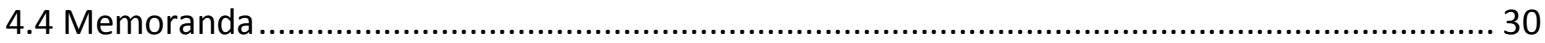

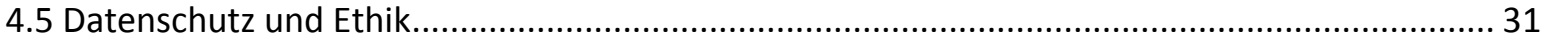




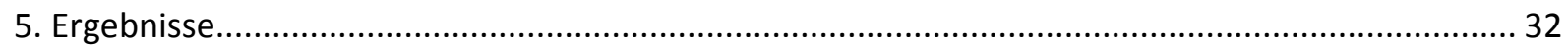

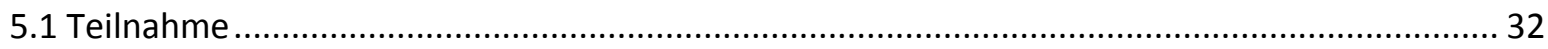

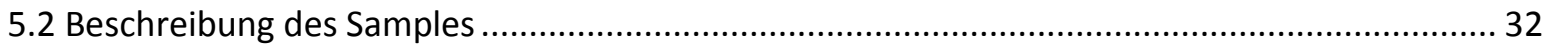

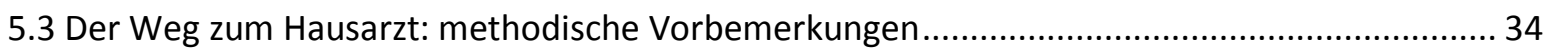

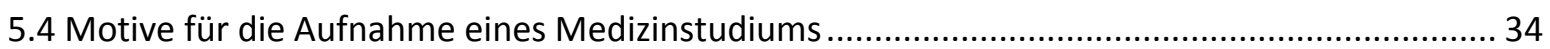

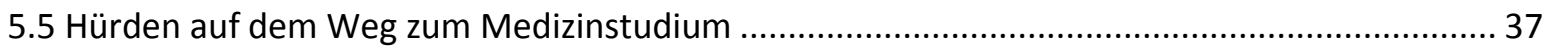

5.6 Die Entscheidung zum „Facharzt für Allgemeinmedizin“ …...................................................... 39

5.7 Motive für die Niederlassung als Hausarzt auf dem Land......................................................... 44

5.8 Hindernisse bei der Niederlassung auf dem Land .................................................................... 48

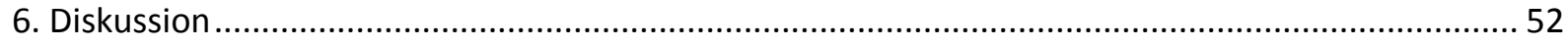

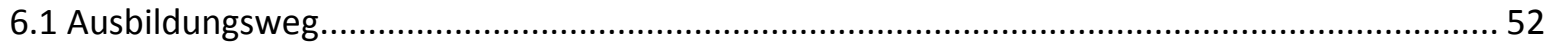

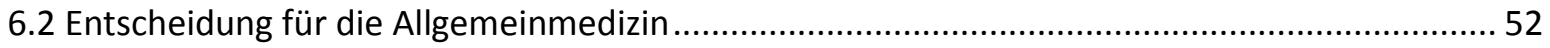

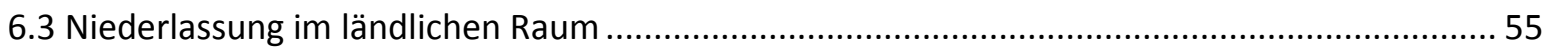

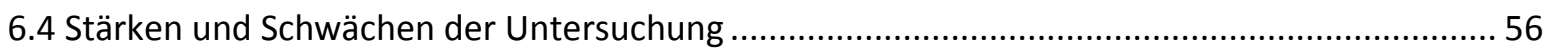

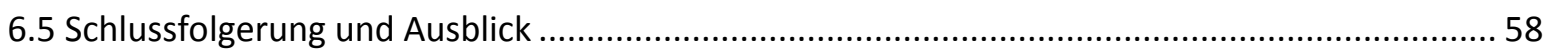

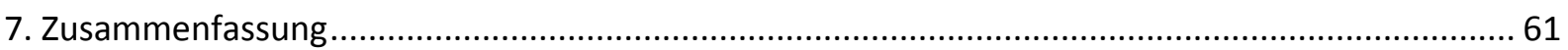

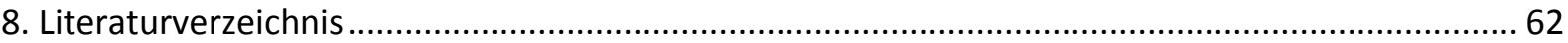

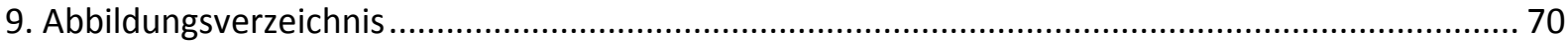

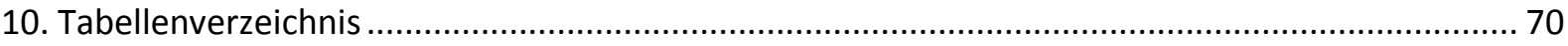

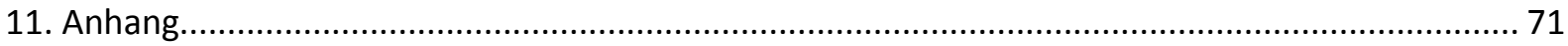

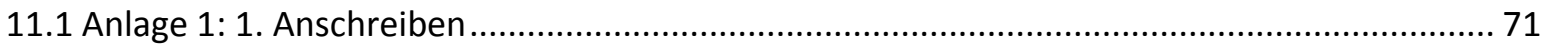

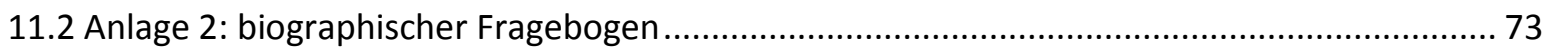

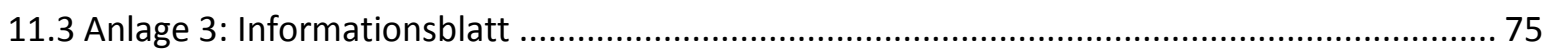

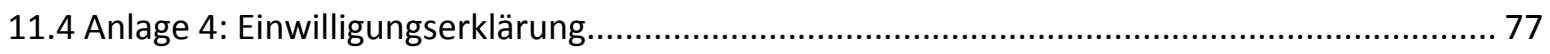

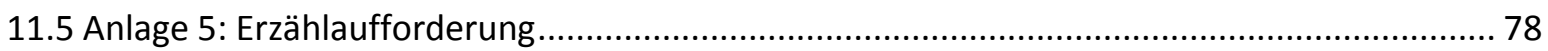

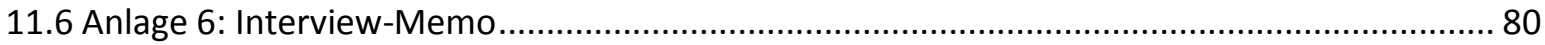

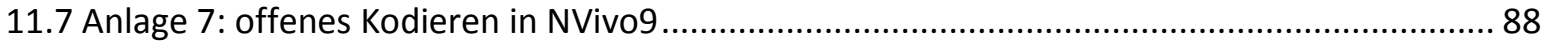

11.8 Anlage 8: Memo entstanden während des Kodierens .......................................................... 89

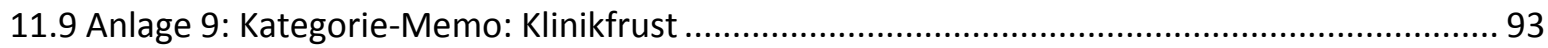

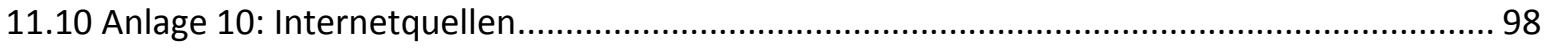




\section{Einleitung}

Dem deutschen Gesundheitswesen gehen die Ärzte und Ärztinnen aus ${ }^{1}$ (BUNDESÄRZTEKAMMER 2002). So oder so ähnlich lauten plakative Aussagen, die uns hinsichtlich der Arztzahlentwicklung in Deutschland eine düstere Zukunft prophezeien.

Besonders die Zahl der Hausärzte hat in den letzten Jahren immer weiter abgenommen: von 54.090 (2001) auf 43.304 (2012). Auch Niedersachsen weist große Lücken in der hausärztlichen Versorgung auf (KOPETSCH 2010, BUNDESÄRTZEKAMMER 2013). Gerade in den ländlichen Regionen scheint dieses Problem allgegenwärtig (BUNDESÄRZTEKAMMER 2002). Den sinkenden Arztzahlen im Hausarztwesen steht eine steigende Zahl an multimorbiden und immobilen Patienten mit einem höheren Bedarf an hausärztlicher Versorgung gegenüber (VAN DEN BERG et al. 2009).

Weltweit laufen Studien und Programme zur Gewinnung zukünftiger Allgemeinärzte. So werden verschiedene Methoden der frühen Heranführung an die hausärztliche Arbeit, insbesondere studienintegrierte Projekte sowie finanzielle Anreize im Studium und bei der Niederlassung diskutiert und erprobt (HALAAS et al. 2008; BÄRNIGHAUSEN und BLOOM 2009; STEGER et al. 2012; KASSENÄRZTLICHE VEREINIGUNG SACHSEN 2009). Trotz intensiver Bemühungen scheinen die Projekte dennoch nicht den gewünschten Effekt hervorzurufen, um den stetig wachsenden Bedarf im ländlichen Bereich decken zu können. So werden beispielsweise die Kapazitäten in Projekten wie der "Studienbeihilfe" - eine finanzielle Unterstützung im Studium verknüpft mit der Verpflichtung, nach dem Studium als Landarzt in unterversorgten Gebieten Sachsens zu arbeiten - nicht ausgeschöpft (KÖHLER 2009).

Neben aussagekräftigen Informationen über den zukünftigen Bedarf an Ärzten sind auch weitere Überlegungen und Strategien wünschenswert, um dem prophezeiten Ärztemangel entgegenwirken zu können (ADLER und KNESEBECK 2011). Diesem Ziel ist auch die vorliegende Dissertation verpflichtet, geht aber - im Vergleich zu bisherigen Bemühungen - einen etwas anderen Weg: nämlich niedergelassene Hausärzte zu fragen, was sie zu einer Niederlassung im ländlichen und kleinstädtischen Bereich veranlasst hat. Die Motive für die Berufswahl „Hausarzt“ sollen mit Hilfe narrativer Interviews herausgearbeitet werden. Auf die-

${ }^{1}$ Zur besseren Lesbarkeit werde ich im weiteren Verlauf dieser Arbeit nur noch die maskuline Form nutzen, soweit es nicht explizit um Geschlechtsunterschiede geht. Selbstverständlich ist die weibliche Form immer mit eingeschlossen. 
ser Grundlage könnte es möglich sein, Ansatzpunkte auch zur Motivation junger Ärzte für solch eine Karriere zu finden, ohne mögliche Probleme und Hürden in diesem Prozess zu verschweigen. 


\section{Stand der Forschung}

Der folgende Überblick über die aktuelle Hausärztesituation in Deutschland präsentiert Forschungsergebnisse über das Niederlassungsverhalten und die bisherigen Strategien zur Erhöhung von Hausärztezahlen. Obschon der Fokus auf deutschen Projekten liegt, werden auch internationale Bemühungen einbezogen.

\subsection{Entwicklungen der Hausarztzahlen mit Schwerpunkt Niedersachsen}

Im weltweiten Vergleich haben nur Belgien, Italien und Griechenland eine höhere Arztdichte als Deutschland (KLOSE et al. 2003). Um eine Überversorgung ${ }^{2}$ in den einzelnen Facharztgebieten zu vermeiden, wird in Deutschland der sogenannte Versorgungsgrad zu Hilfe genommen. Dieser errechnet sich aus dem Verhältnis von Einwohnerzahlen zu Arztzahlen. Liegt der erhaltene Wert über $110 \%$, so wird das jeweilige Facharztgebiet für eine weitere Niederlassung gesperrt (KLOSE et al. 2007). Insgesamt liegt der Versorgungsgrad bezogen auf alle Vertragsärzte in Gesamtdeutschland bei 126,5 \% (KLOSE und REHBEIN 2011). Mit Blick auf die verschiedenen Planungsbereiche stellten die Autoren eine Überversorgung in 182 von 395 Planungsbereichen fest. Doch auf dem Land sind Engpässe in der ärztlichen Versorgung vorhanden. Von einer echten Unterversorgung sprechen KLOSE et al. (2007) für den Saalkreis in Sachsen-Anhalt. Für Hausärzte wird hier ein Versorgungsgrad von lediglich 64,8 \% erreicht (KLOSE und REHBEIN 2011). Insgesamt liegt der Versorgungsgrad der Hausärzte in SachsenAnhalt jedoch mit 93 \% nur knapp unter der 100 \%-Marke, die in allen anderen Bundesländern überschritten wird (KLOSE und REHBEIN 2011 vgl. Abbildung 1). Hamburg, Bremen und Berlin haben unter Berücksichtigung aller Arztgruppen die höchste Arztdichte. Schlusslichter im nationalen Vergleich sind Brandenburg, Sachsen-Anhalt und Niedersachsen.

2 „Überversorgung ist anzunehmen, wenn der allgemeine bedarfsgerechte Versorgungsgrad um 10 von Hundert überschritten ist. Der allgemeine bedarfsgerechte Versorgungsgrad ist erstmals bundeseinheitlich zum Stand vom 31. Dezember $1990 \mathrm{zu}$ ermitteln. Bei der Ermittlung des Versorgungsgrades ist die Entwicklung des Zugangs zur vertragsärztlichen Versorgung seit dem 31. Dezember 1980 arztgruppenspezifisch angemessen zu berücksichtigen. Die regionalen Planungsbereiche sind mit Wirkung zum 1. Januar 2013 so festzulegen, dass eine flächendeckende Versorgung sichergestellt wird" (SOZIALGESETZBUCH $\mathrm{V}, \S 101)$. 


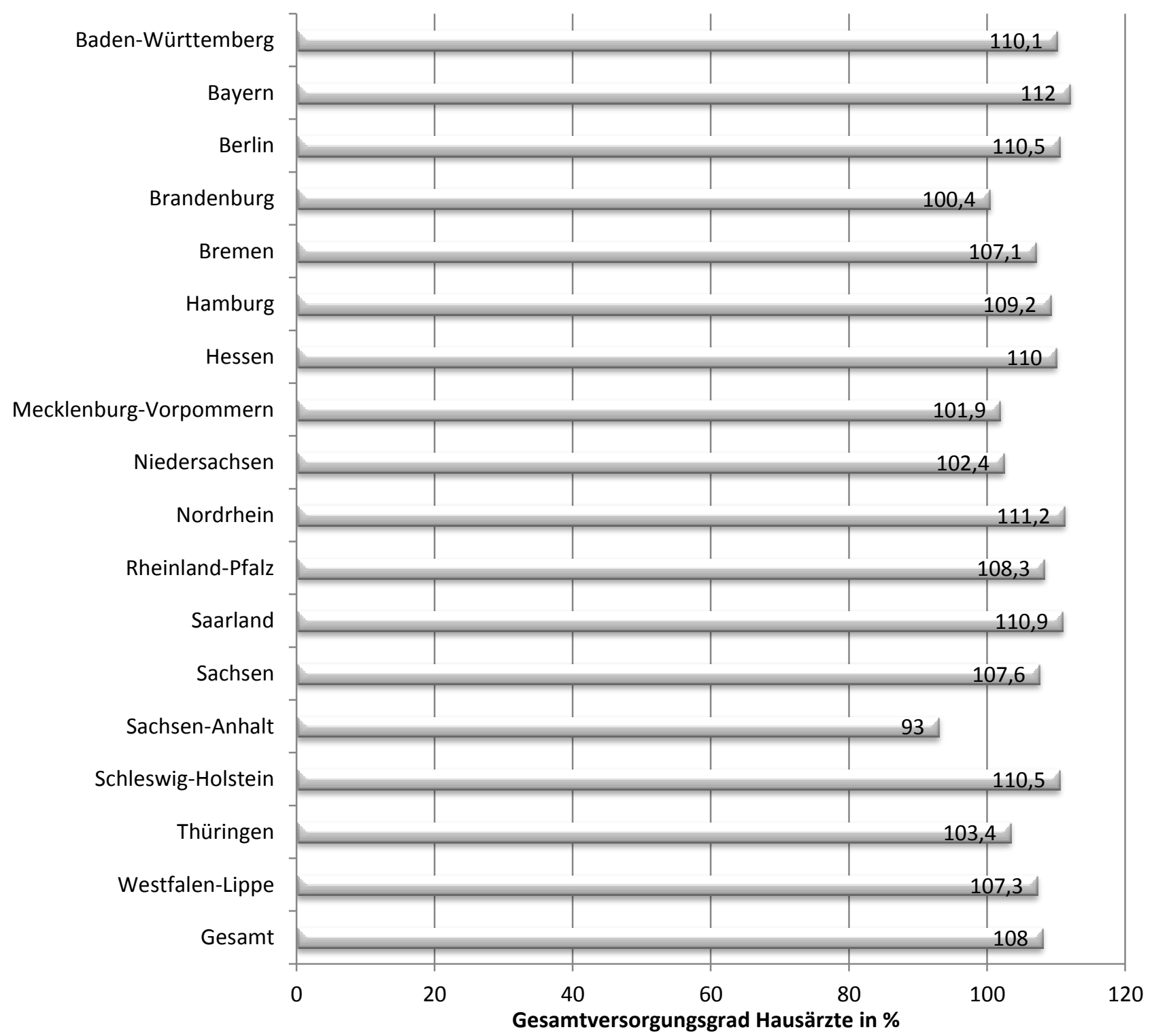

Abbildung 1 Gesamtversorgungsgrad mit Hausärzten nach KVen 2010; nach KLOSE und REHBEIN 2011, S.12

Betrachtet man die Niederlassungszahlen der Vertragsärzte in Deutschland zwischen 1990 und 2009, gibt es eine Zunahme um 34,4 \% von 88.811 auf 135.434 (KLOSE und REHBEIN 2011). Die Zunahme liegt hauptsächlich auf Seiten der Fachärzte, hier hat sich die Zahl bis 2001 um 44,5 \% erhöht, die Zahl der Allgemeinärzte/praktischen Ärzte nur um 12,3 \% (KLOSE et al. 2003).

Für die meisten Fachärzte sind die Planungsbereiche in Niedersachsen gesperrt. Lediglich bei den Hausärzten gibt es vermehrt offene Planungsstellen (KASSENÄRZTLICHE VEREINIGUNG NIEDERSACHSEN, 2013). Das gleiche Bild zeigt sich auch im gesamtdeutschen Vergleich (vgl. KLOSE et al. 2003, Abbildung 5). Aus diesen Daten wird ersichtlich, dass es in Deutschland 
ein massives Verteilungsproblem von Vertragsärzten gibt: Städtische Gebiete sind noch ausreichend versorgt, der ländliche Bereich weist aber schon jetzt große Lücken auf. Insgesamt ist die Vertragsarztdichte in Deutschland jedoch auf einem hohen Niveau (KLOSE et al. 2003). 2009 wurde ein Höchststand mit 397 berufstätigen Ärzten pro 100.000 Einwohnern erreicht (KLOSE und REHBEIN 2011).

Bei den Hausärzten zeigt sich jedoch ein anderes Bild. Bereits 2009 fehlten in Niedersachsen mindestens 219 Hausärzte (KASSENÄRZTLICHE BUNDESVEREINIGUNG 2010). Auch die Altersentwicklung der Hausärzte wirkt sich negativ auf die zukünftige Hausarztversorgung aus. Ende 2012 waren 71,7 \% von ihnen mindestens 50 Jahre alt (BUNDESÄRZTEKAMMER 2013). Bis zum Jahr 2020 müssten 23.768 Hausärzte ersetzt werden. Dementsprechend ist bis 2020 mit einem Hausärzterückgang von 13,3 \% zu rechnen (KOPETSCH 2010).

Der Ärztenachwuchs kann dieses Defizit nicht auffangen. Seit 2007 ist die Zahl der Studierenden im ersten Semester um 2.500 Studierende zurückgegangen, nachdem zwischen 1993 bis 2006 die Zahl der Studierenden mit etwa 12.100 relativ konstant geblieben war (KOPETSCH 2010). Des Weiteren stieg parallel die Zahl der Studienabbrecher und Studienwechsler an. So ist ein Rückgang der Studierendenzahlen von 13,6 \% auf 76.042 zwischen den Jahren 1994 und 2008 zu verzeichnen. Berücksichtigen muss man dabei die Novellierung der Approbationsordnung, die zu einer Verringerung der Studienplätze führte. So lassen sich etwa 5 \% des Rückgangs erklären. In den Jahren 2007 und 2008 hingegen ist ein kurzfristiger Anstieg der Absolventenzahlen auf die Änderung der Approbationsordnung und den damit verbundenen statistischen Effekten zurückführen. So versuchten einige der Langzeitstudierenden, ihr Studium noch nach den Kriterien der 1987 beschlossenen Approbationsordnung zu beenden. Insgesamt entsprechen die Verluste etwa der Größe eines kompletten Anfängerjahrganges (KOPETSCH 2010).

Auch nach erfolgreicher Approbation gehen dem deutschen Gesundheitssystem Ärzte verloren. So haben sich im Jahr 2008 nur 81,6\% der Studierenden mit bestandener ärztlicher Prüfung bei der deutschen Ärztekammer erstangemeldet. Die übrigen approbierten Mediziner sind somit nie als Arzt klinisch tätig geworden oder in das Ausland abgewandert (KOPETSCH 2010). 
Nach einem Anstieg in den Jahren 2000 ff. ist seit 2007 die Zahl der Gebietsanerkennungen für Allgemeinmedizin von 1.938 auf 1.168 gefallen (vgl. KOPETSCH 2010, Abb. 4.9). Auffällig ist zugleich, dass über den ganzen Zeitraum hinweg die Zahl der Niederlassungen hinter der Zahl der Gebietsanerkennungen zurückbleibt (vgl. KOPETSCH 2010, Abb. 4.10). Laut KOPETSCH ist „die Differenz zwischen den erteilten Gebietsanerkennungen und den Niederlassungen im Fach Allgemeinmedizin in erster Linie auf einen Ärztinnenschwund zurückzuführen“ (KOPETSCH 2010, S. 62). Wenn man berücksichtigt, dass zudem der Frauenanteil bei den berufstätigen Ärztinnen von 1991 bis 2012 von 33,6 \% auf 44,3\% angestiegen ist, so hat die „Feminisierung“ der Medizin einen entscheidenden Einfluss auch auf die Entwicklung der Hausärztezahlen in Deutschland (KOPETSCH 2010, BUNDESÄRZTEKAMMER 2013). In Zukunft wird sich dieser Trend zudem noch verschärfen; bereits jetzt beträgt der Frauenanteil unter den Medizinstudierenden 62 \% (HIBBELER 2013). Dieser Prozess führt zu einer mehrheitlich weiblichen Ärztegeneration, welche die zukünftigen Hausärzte hervorbringen wird, obschon zu vermuten ist, dass diese Ärztinnen auf dem Weg zum Facharzt der Allgemeinmedizin wieder vorrübergehend, vielleicht auch für immer, verloren gehen, wenn nicht eine Verbesserungen in der Vereinbarkeit von Familie und Beruf herbeigeführt werden kann. Denn laut KOPETSCH (2010) ist die schlechte Vereinbarkeit von Familie und Beruf Hauptursache für den Ärztinnenschwund.

\subsection{Einflussfaktoren auf die Berufs- und Niederlassungsentscheidung}

Zum Thema Hausärztemangel liegen einige Studien vor, die sich mit den Einflussfaktoren auf eine Niederlassung befasst haben. Allerdings stammen die meisten dieser Studien aus den USA oder Kanada, da auch dort in den ländlichen Bereichen ein Hausärztemangel herrscht. Die Übertragbarkeit dieser Studien auf Deutschland ist allerdings begrenzt. Bereits die Definition „ländlicher Bereich“ fällt in Kanada aufgrund geographischer Gegebenheiten ganz anders aus. So leben auf einer Fläche von 9.984.670 km² 34.483.000 Menschen, in Deutschland sind es hingegen mehr als doppelt so viele Menschen (81.726.000) bei einer Fläche von nur $357.121 \mathrm{~km}^{2}$ (FISCHER WELTALMANACH 2012). Auch die Gesundheitsversorgung weist Unterschiede auf. So hat man in den USA beispielsweise keine freie Arztwahl bei den Hausärzten und Fachärzten, in Kanada keine freie Wahl bei den Fachärzten. Die Krankenhauswahl ist in Kanada eingeschränkt und in den USA nicht verfügbar (BESKE et al. 2005). Diese Grenzen für die Übertragbarkeit sollte man berücksichtigen, wenn auch das große Interesse an einer Verbesserung der Hausarztversorgung Ideen für Deutschland anregen könnte: 
Die persönliche Einstellung zum Landleben dominiert in einer amerikanischen Studie von DANIELS et al. (2007) die Entscheidung, auf dem Land tätig zu werden. Selbst eine Kindheit auf dem Land verlebt zu haben, wirkt sich ebenfalls positiv auf die Entscheidung aus (HALAAS et al. 2008 und LU et al. 2008).

In Studien zur Zufriedenheit von Hausärzten bedeutete besonders der Mangel an Zeit für Familie und Freizeitaktivitäten eine große Entbehrung für die Ärzte (BOVIER und PERNEGER 2003). Gerade deshalb mag auch die eigene Familie ein wichtiger Einflussfaktor in diesem Zusammenhang sein. So ist Ärzten wichtig, dass auch der Lebenspartner privat und beruflich im ländlichen Bereich Fuß fassen kann. Ebenso wichtig für die Entscheidung zu einer hausärztlichen Niederlassung scheinen die Ausbildungsmöglichkeiten der Kinder sowie die räumliche Nähe zu weiteren Angehörigen und Freunden (HENRY et al. 2009).

Da auch die vergleichsweise geringe Entlohnung sich negativ auf die Berufsentscheidung auswirkt (BOVIER und PERNEGER 2003), könnten finanzielle Anreize die hausärztliche Niederlassung fördern. So gibt es zum einen Stipendien oder Studienkredite mit Leistungsanforderungen und Kreditrückzahlungsprojekten, zum anderen direkte finanzielle Entschädigungen. Die Aufwandsentschädigungen für ein Jahr Arbeit im ländlichen Bereich variierten dabei erheblich (BÄRNIGHAUSEN und BLOOM 2009). Sowohl die Teilnahme an den oben genannten Programmen als auch der Erhalt einer direkten finanziellen Förderung begünstigten die Niederlassung in ländlichen Gebieten. BÄRNIGHAUSEN und BLOOM (2009) wollten aber in ihrem Review nicht ausschließen, dass der signifikante Unterschied nicht bereits auf die Vorauswahl der Programmteilnehmer zurückzuführen ist.

\subsection{Internationale Bemühungen zur Erhöhung der Hausarztzahlen}

Trotz der bereits genannten Grenzen der Übertragbarkeit möchte ich auf zwei Projekte aus dem Ausland ausführlicher eingehen, um ein vollständigeres Bild der Forschungen und Bemühungen zur Förderung der primärärztlichen Versorgung zu entwickeln.

Der Staat Minnesota, einer der Vorreiter auf diesem Gebiet, bemüht sich bereits seit den späten sechziger Jahren des 20. Jahrhunderts, Medizinstudierende für eine Karriere auf dem Land zu gewinnen. In diesem Zusammenhang etablierte die University of Minnesota zwei Programme: den „Duluth Campus“ der Medical School of Minnesota (2.3.1) und das „Rural Physician Associated Program“(2.3.2). 


\subsubsection{Medical School Program in Duluth}

Gegründet wurde das Medical School Program in Duluth 1969 als eine Abspaltung der University of Minnesota. 1972 startete das Programm mit 24 Studierenden (ZINK et al. 2010). Vier Jahre später machten die ersten 23 ihren Abschluss am Duluth Campus. Ziel des Programmes ist es, neue Hausärzte zu gewinnen, die sich um die Primärversorgung in ländlichen Bereichen Minnesotas und in den Indianercamps kümmern (UNIVERSITY OF MINNESOTA ACADEMIC HEALTH CENTRE 2010). Jährlich werden dafür mittlerweile durchschnittlich 60 Studierende ausgebildet. Diese Ausbildung dauert zwei Jahre, danach haben die Studierenden verschiedene Möglichkeiten, ihre medizinische Ausbildung zu Ende zu bringen: z.B. in das Rural Physician Associated Program einzutreten (UNIVERSITY OF MINNESOTA ACADEMIC HEALTH CENTRE 2010).

Nach bereits acht Wochen in der Medical School erhalten die Studierenden einen ärztlichen Mentor. Mit diesem treffen sie sich zehnmal während des Jahres. Die Treffen finden zu unterschiedlichen Tageszeiten statt, um so unterschiedliche Einblicke in die Arbeit von Hausärzten zu erhalten. Um auch ein Verständnis für den Tagesablauf der Ärzte, ihr Familienleben und das Leben in einer ländlichen Gemeinde zu erhalten, gehen die Studierenden am Ende des ersten Semesters und einige Male während des zweiten Semesters für jeweils drei Tage in die Familien der Ärzte. Die Familien leben alle in ländlichen, medizinisch unterversorgten Bereichen Minnesotas und Wisconsins (ZINK et al. 2010).

Das Programm wurde 1990 für seine Förderung der medizinischen Versorgung mit dem National Rural Health Association's National Outstanding Rural Health Program Award ausgezeichnet (ZINK et al. 2010).

\subsubsection{Rural Physician Associated Program}

Das Rural Physician Associated Program ist ein Programm für Medizinstudierende nach dem dritten Jahr Medical School. Sowohl Studierende vom Duluth Campus als auch Studierende des Twin City Campus der University of Minnesota dürfen sich für dieses Projekt bewerben. Gegründet wurde das Programm im Jahre 1971 (UNIVERSITY OF MINNESOTA 2013). Jedes Jahr nehmen an dem Programm, das durch den Staat Minnesota finanziert wird, durchschnittlich 33 Studierende teil. 
Für neun Monate werden Studierende in einer ländlichen Gemeinde aufgenommen, in denen sie einem ärztlichen Ansprechpartner zugeteilt sind. Da insgesamt 110 Gemeinden an dem Programm teilnehmen, kann sichergestellt werden, dass immer nur ein Studierender einer Gemeinde zugeteilt wird und ein intensiver Einblick in die medizinische Versorgung auf dem Land gewährt wird. Durch die neunmonatige Dauer des Programms lernen die Studierenden, Patienten über einen langen Zeitraum zu beobachten und Krankheitsentwicklungen einzuschätzen (HALAAS et al. 2008).

\subsubsection{Ergebnisse des Medical School Program in Duluth und des RPAP}

Nach ZINK et al. (2010) bestärken beide Projekte die Wahl, später im hausärztlichen Bereich auf dem Land tätig zu werden. Diese Aussage beruht auf den Daten von 3.365 Studierenden: 215 von ihnen haben erst das Programm in Duluth durchlaufen und anschließend das RPAP, 276 waren zuerst auf der herkömmlichen Medical School und sind anschließend ins RPAP gewechselt, weitere 427 haben nur am Duluth Programm teilgenommen und haben sich danach für die herkömmliche Medical School entschieden, die restlichen 2.477 haben gänzlich die Medical School der University of Minnesota durchlaufen (ZINK et al. 2010).

Im Ergebnis haben $34 \%$ derer, die entweder am Duluth Program oder am RPAP teilgenommen haben, den Weg zum „Primary Care Physician“ ${ }^{3}$ eingeschlagen. Das sind weit mehr als der nationale Standard, der im Jahre 2002 bei 5 \% lag. Bei den Studierenden, die beide Programme durchlaufen haben, entschieden sich sogar 54 \% für eine Arbeit als „Primary Care Physician“ (RABINOWITZ und PAYNTER 2002).

\subsection{Bemühungen zur Erhöhung der Hausarztzahlen in Deutschland}

Auch in Deutschland gibt es verschiedene Ansätze, die den vorausgesagten Ärztemangel in der Allgemeinmedizin verhindern oder zumindest vermindern sollen. Nachfolgend werde ich verschiedene aktuelle Ansätze vorstellen und näher beleuchten. Diese sind Beispiele für die praktische Umsetzung der im Kapitel 2.2 beschriebenen Ansätze.

\subsection{1 „Klasse Allgemeinmedizin“}

Die „Klasse Allgemeinmedizin“ ist ein Projekt der Martin-Luther-Universität HalleWittenberg, das erstmalig im Wintersemester 2011/2012 stattfand. Das Projekt wird von

3 Primary Care Physcian beinhaltet: „internal medicine, pediatrics, internal medicine/pediatrics, or family medicine." Internisten und Kinderärzte mit Fachweiterbildung werden den Spezialisten zugeordnet. 
drei „Klassenlehrern“ (STEGER et al. 2012, S. 267) sowie einem „persönlichen landärztlichen Mentor" (S. 267) begleitet. Sowohl Studierende der vorklinischen als auch klinischen Abschnitte können an diesem Wahlpflichtfach mit einem Leistungsumfang von einer Semesterwochenstunde teilnehmen. Von insgesamt etwa 200 Medizinstudierenden in Halle bewarben sich im Oktober 2011 etwa 40 Studierende, mit einem vergleichsweise hohen Männeranteil von 55 \% (bundesweit 37 \%). Diese Tatsache lässt STEGER spekulieren, ob das Projekt - über den eigentlichen Nutzen hinaus - auch der Entwicklung der Allgemeinmedizin hin zu einem Frauenfach (JACOB et al. 2010, S. 24) entgegenwirkt. Von einer Vorauswahl von Studierenden wurden dann 20 letztendlich aufgenommen. Auswahlkriterien waren unter anderem Interesse der Studierenden an der Allgemeinmedizin, ausreichendes Durchhaltevermögen und ein kleinstädtischer beziehungsweise ländlicher Hintergrund. Dieses letzte Kriterium musste nicht zwangsläufig erfüllt sein.

Die ausgewählten 20 Studierenden werden während ihres kompletten Studiums zwei Tage pro Semester in einer ländlichen allgemeinärztlichen Praxis verbringen. Hinzu kommen pro Semester jeweils drei 90-minütige Seminare, die eine theoretische Vorbereitung auf die Praxistage gewährleisten sollen. Diese Theorieseminare beinhalten sowohl ein „Kommunikationstraining", ein „Fertigkeitentraining" sowie „Fallreflexionen“ (STEGER et al. 2012, S. 266) in Balintgruppen. Schwerpunkte liegen dabei besonders auf dem Erlernen von Anamnesen sowie der klinischen Untersuchung. Aber auch dem betriebswirtschaftlichen Aspekt einer Praxisführung wird Aufmerksamkeit geschenkt. Durch dieses Projekt wird versucht, den Studierenden zu vermitteln, den Patienten in seiner „biopsychosozialen Gesamtheit wahrzunehmen“ (STEGER et al. 2012, S. 266) und dieses Konzept in seiner „praktischen Relevanz“ (S. 266) zu erleben. Zudem erhoffen sich die Projektgründer eine stärkere hausärztliche Identitätsbildung unter den Studierenden.

\subsubsection{Summerschool Allgemeinmedizin}

Um den Nachwuchs der Allgemeinmedizin zu fördern, richtete die Nachwuchsakademie der Deutschen Gesellschaft für Allgemeinmedizin und Familienmedizin (DEGAM) im Sommer 2014 bereits zum vierten Mal eine Allgemeinmedizinische Summerschool aus. Gefördert wird das Projekt durch den Deutschen Hausärzteverband und die Gesellschaft der Hochschullehrer für Allgemeinmedizin e.V. (DEGAM 2014). Teilnehmen können alle Medizinstudierenden aus Deutschland, die bereits den klinischen Studienabschnitt erreicht haben (DE- 
GAM 2013). 2013 bestand das Team aus 17 Studentinnen und 8 Studenten zwischen 22 und 30 Jahren. Der Anteil der Männer konnte damit im Vergleich zum Vorjahr von 8 \% auf 32 \% erhöht werden (BOJANOWSKI et al. 2013). Trotzdem lässt sich offensichtlich auch hier die „Feminisierung“ der Medizin ablesen.

Die Summerschool hat sich zur Aufgabe gemacht, einen tieferen Einblick in die Arbeit der Hausärzte zu gewährleisten. So standen für den Sommer 2013 Themen wie „Was machen Hausärzte?“, „Wie werde ich Hausarzt?“, aber auch Untersuchungs-, Entspannungs- und Kommunikationskurse auf dem Programm (DEGAM 2013, S. 2). Ein Teilnehmer beschrieb die Summerschool als einen „faszinierenden Tauchgang in die vielfältige Welt der Allgemeinmedizin“ (BOJANOWSKI et al. 2013, S. 118). Für das Frühjahr 2014 ist erstmalig auch eine Winterschool mit dem Thema „Perspektive Hausarzt“ geplant (HAUSÄRZTEVERBAND BADENWÜRTTEMBERG 2013).

\subsubsection{Programm „Studienbeihilfe“}

Im Bundesland Sachsen haben die Kassenärztliche Vereinigung, einige Krankenkassen (AOK PLUS, Ersatzkassen, BKK MEDICUS, IKK Sachsen, Knappschaft) und das Staatsministerium für Soziales und Verbraucherschutz 2008 das Gemeinschaftsprojekt „Studienbeihilfe“ ins Leben gerufen. Jährlich haben 50 Medizinstudierende, die an einer deutschen Medizinischen Fakultät eingeschrieben sind, die Möglichkeit, diese spezielle Förderung zu beantragen. Die Beantragung kann erst nach dem Ersten Abschnitt der Ärztlichen Prüfung erfolgen und setzt ein Studium in Regelstudienzeit mit 1/2-jährlicher Überprüfung durch die Kassenärztliche Vereinigung voraus. Die Studierenden erhalten für maximal 48 Monate eine finanzielle Förderung von monatlich $300 €$ im ersten und zweiten Jahr, $400 €$ im dritten und $600 €$ im vierten Jahr. Voraussetzung für die Beantragung der Förderung ist die vertragliche Einwilligung, dass die Studierenden sich während des Studiums eine Patenschaftspraxis suchen. Diese Patenschaftspraxis muss im Bereich der Kassenärztlichen Vereinigung Sachsen liegen und erfordert eine fachspezifische Begleitung der Studierenden an mindestens einem Tag pro Monat.

Unmittelbar nach der erfolgreichen Beendigung des Studiums muss der Studierende eine Fachweiterbildung Allgemeinmedizin oder eine andere Fachweiterbildung, die zur Niederlassung als Hausarzt befähigt, beginnen. Im Anschluss verpflichtet er sich, für vier Jahre in einem unterversorgten Planungsbereich Sachsens eine volle, mindestens jedoch 75 \%-ige Hausarztstelle zu übernehmen. Es besteht die Möglichkeit einer eigenen Niederlassung oder 
die Übernahme einer Angestellten-Position im hausärztlichen Bereich. Werden die Auflagen nicht erfüllt, muss der erhaltene Betrag zuzüglich eines 5 \%-igen Zinssatzes zurückgezahlt werden (KASSENÄRZTLICHE VEREINIGUNG SACHSEN 2009). Das Projekt läuft vorerst bis einschließlich 2012/2013; über den weiteren Verlauf des Projektes sind noch keine Angaben zu finden (KASSENÄRZTLICHE VEREINIGUNG SACHSEN 2012).

\subsubsection{Pflichttertial im PJ}

Im Jahr 2002 wurde mit Änderung der Approbationsordnung § 3 Abs. 1 die Möglichkeit zur Absolvierung eines PJ-Tertials in der Allgemeinmedizin geschaffen (APPROBATIONSORDNUNG FÜR ÄRZTE 2013). Nur etwa 12-15 \% der Studierenden entscheiden sich derzeitig für ein PJ-Tertial in der Allgemeinmedizin (BUNDESMINISTERIUM FÜR GESUNDHEIT 2011 b). Um diesem Problem entgegenzuwirken, wurde im Bundesrat über einen verpflichtenden Ausbildungsabschnitt in der Allgemeinmedizin während des Medizinstudiums diskutiert (BUNDESMINISTERIUM FÜR GESUNDHEIT 2012 a). Da jedoch alle maßgeblichen Ärztevertreter und die Studierenden ein verpflichtendes PJ-Tertial in der Allgemeinmedizin ablehnten, wurde am 11. Mai 2012 die neue Approbationsordnung mit Auflagen akzeptiert (BUNDESMINISTERIUM FÜR GESUNDHEIT 2012 a). Um die Allgemeinmedizin nachhaltig zu stärken, ist in der neuen Approbationsordnung eine Mindestdauer des Blockpraktikums in der Allgemeinmedizin von zwei Wochen verankert (APPROBATIONSORDNUNG FÜR ÄRZTE 2013). Die Gesetzesänderung ist zum Oktober 2013 in Kraft getreten (BUNDESMINISTERIUM FÜR GESUNDHEIT 2012 b). Auch die Anzahl der PJ-Plätze in der Allgemeinmedizin soll mit Wirkung ab Januar 2014 auf langfristig 20 \% erhöht werden (BUNDESMINISTERIUM FÜR GESUNDHEIT 2011 b).

\subsubsection{Verbundweiterbildung}

In Kanada, den USA, Skandinavien und Großbritannien läuft ein Großteil der Weiterbildung zum Allgemeinarzt bereits seit Jahrzehnten über Weiterbildungsverbünde (DONNERBANZHOF et al. 2009). Auch in Deutschland erkennt man nun die Notwendigkeit zur Schaffung dieser Strukturen. Das erfordert einen nahtloser Übergang zwischen verschiedenen Gebieten, die der Ausbildung in der Allgemeinmedizin dienlich sind, durch Verträge oder verbindliche Zusagen (DONNER-BANZHOF et al. 2009). Ziel dieser allgemeinmedizinischen Weiterbildungsverbünde ist es, den Weg in die Allgemeinmedizin zu erleichtern und so weniger Ärzte an der Hürde der Weiterbildung zu verlieren. 
Insgesamt dauert die Weiterbildung im Fach Allgemeinmedizin fünf Jahre und ist in den Weiterbildungsverordnungen der Landesärztekammern geregelt. Für Niedersachsen sind dies: „60 Monate bei einem Weiterbildungsermächtigten an einer Weiterbildungsstätte gemäß $\S$ 6 Abs. 1 Satz 1, davon

- 36 Monate in der stationären Basisweiterbildung im Gebiet Innere Medizin, davon können bis zu

○ 18 Monate in den Gebieten der unmittelbaren Patientenversorgung (auch 3Monats-Abschnitte) auch im ambulanten Bereich angerechnet werden,

- 24 Monate Weiterbildung in der ambulanten hausärztlichen Versorgung, davon können bis zu

○ 6 Monate in Chirurgie (auch 3-Monats-Abschnitte) angerechnet werden “

\section{(ÄRZTEKAMMER NIEDERSACHSEN 2013, S. 14).}

Hieran wird deutlich, dass die Weiterbildungsordnung stark auf die Innere Medizin ausgerichtet ist. Für die Verbundweiterbildung hat die DEGAM eine Empfehlung herausgegeben, die eine Fachbereichszuteilung in drei Gruppen vorsieht. Die Gruppereihenfolge entspricht einer Rangreihenfolge verschiedener Fachrichtungen, die während der Verbundweiterbildung durchlaufen werden sollten (DONNER-BANZHOF et al. 2009 ). So umfasst die zentrale erste Gruppe die Bereiche

- Allgemeinärztliche Praxis

- Innere Medizin (v.a. Kardiologie, Gastroenterologie, Diabetologie)

- Chirurgie (v.a. Viszeralchirurgie, Traumatologie, Ambulanz)

- Kinderheilkunde.

Die zweite, wesentliche Gruppe beinhaltet

- Dermatologie

- Psychiatrie

- Psychosomatik-Psychotherapie

- Orthopädie und Unfallchirurgie

- Hals-Nasen-Ohren-Erkrankungen.

Die Bereiche der letzten Gruppe werden als durchaus nützlich dargestellt. Hierunter fallen 
- Urologie

- Neurologie

- Gynäkologie und Geburtshilfe

- Augenheilkunde

- Anästhesie

- Arbeitsmedizin

- Geriatrie

- Rehabilitative Medizin.

Eine Verbundweiterbildung muss jedoch nicht alle Fächer anbieten können (DONNERBANZHOF et al. 2009).

Für die in die Verbundweiterbildung aufgenommenen Ärzte gibt es einen persönlichen Mentor. Bei diesem sollte es sich möglichst um einen erfahrenen Facharzt handeln, der selbst ausgewählt werden darf und ebenfalls zur Erhöhung der Qualität und Strukturierung beitragen soll (DONNER-BANZHOF et al. 2009). Auch bei der Frage zur Ausbildung der weiterbildungsbefugten Ärzte bietet das Programm „Verbundweiterbildung plus“ eine Lösung. So bot es erstmalig einen „Train the Trainer“ - Workshop im Juli 2011 an (STEINHÄUSER et al. 2012). Eine Expertenkommission aus England, Dänemark und den Niederlanden hatte zuvor im Jahr 2009 den Mangel an einem solchen Programm beklagt, da Deutschland so in keinem Falle den europäischen Standard in der Allgemeinärzte - Weiterbildung erfüllen würde (ANNAN et al. 2010). Die gezielte Ausbildung der weiterbildungsbefugten Ärzte soll die Qualität der Ausbildung deutscher Allgemeinärzte weiter steigern.

\subsubsection{Weiterbildungszuschuss}

Seit 1999 sind die gesetzlichen Krankenkassen zu einer Zuschusszahlung für Assistenten in der Weiterbildung zum Allgemeinarzt verpflichtet (DONNER-BANZHOF et al. 2009). Jährlich können damit ca. 5.000 Weiterbildungsstellen gefördert werden. Es können sowohl Halb- als auch Ganztagsstellen gefördert werden (VEREINBARUNG VON DKG, KBV und GKV 2008).

Die Förderung beträgt nach §4 der Vereinbarung zur Förderung der Weiterbildung in der Allgemeinmedizin in der ambulanten und stationären Versorgung im ambulanten Bereich 1.750 $€$ pro Monat, die jedoch je nach zuständiger Kassenärztlicher Vereinigung bis auf $3.500 €$ pro Monat aufgestockt werden kann. In hausärztlich unterversorgten Gebieten wird dieser Be- 
trag um weitere $500 €$ aufgestockt. Auch während der Weiterbildung im stationären Bereich können Zuschüsse bezogen werden (VEREINBARUNG VON DKG, KBV und GKV 2008).

Mit Hilfe dieser Förderung soll die Attraktivität der hausärztlichen Weiterbildung gesteigert werden.

\subsubsection{Versorgungsstrukturgesetz}

Die Überschrift der Pressemitteilung des Bundesministeriums für Gesundheit zum neuen Versorgungsstrukturgesetz lautet: „Eine flächendeckende bedarfsgerechte medizinische Versorgung bleibt auch in Zukunft in ganz Deutschland gesichert“ (BUNDESMINISTERIUM FÜR GESUNDHEIT 2011 a, S.1). Das Versorgungsstrukturgesetz wurde am 01. Dezember $2011 \mathrm{im}$ Deutschen Bundestag erlassen und soll, wie man bereits der Überschrift entnehmen kann, eine „langfristig qualitativ hochwertige medizinische Versorgung" gewährleisten, so der damalige Bundesgesundheitsminister Bahr (BUNDESMINISTERIUM FÜR GESUNDHEIT 2011 a, S. 1).

Um den Bereich der hausärztlichen Versorgung attraktiver zu gestalten, gab es Änderungen im Vergütungssystem. Für Ärzte sollen in unterversorgten Gebieten keine Mengenbegrenzungen mehr gelten, dementsprechend würden auch alle von ihnen erbrachten Leistungen voll vergütet werden. Mit Hilfe von Strukturfonds der Kassenärztlichen Vereinigungen soll ebenfalls eine Niederlassung in ländlichen Regionen begünstigt werden. Bis zu 0,1 \% der morbiditätsbedingten Vergütung der Kassenärztlichen Vereinigungen könnte hierfür genutzt werden. Die Krankenkassen müssten in einem solchen Fall den Fond zum gleichen Teil mitfanzieren. Durch diese Fonds könnten somit beispielsweise Kosten, die durch eine Niederlassung für den Arzt anfallen, gedeckt werden. Aber auch Stipendien im Bereich der Nachwuchsgewinnung sind hierdurch denkbar (BUNDESGESETZBLATT GKV-VStG, 2011).

Von nun an sind Kassenärztliche Vereinigungen auch in der Lage, unbesetzte Praxen in strukturschwachen Regionen selber zu übernehmen oder an Kommunen zu übertragen. Dies soll die Wiederbesetzung der Praxis erleichtern (BUNDESGESETZBLATT GKV-VStG, 2011).

Auch die Familienfreundlichkeit wurde durch das neue Gesetz verstärkt. Ärzte können sich jetzt doppelt so lange, also insgesamt zwölf Monate, nach der Geburt eines Kindes vertreten lassen. Danach können sie zudem insgesamt 36 Monate einen Entlastungsassistenten beschäftigen (BUNDESGESETZBLATT GKV-VStG, 2011). 
Ein weiterer Faktor, der die Attraktivität des Hausarztwesens steigern soll, ist die Beratung in wirtschaftlichen Fragen durch die Kassenärztliche Vereinigung. Größere Transparenz im Arzneimittelsektor soll zugleich einen Schutz vor gefürchteten Regressforderungen bieten (BUNDESGESETZBLATT GKV-VStG, 2011).

\subsection{Zusammenfassung}

Das Problem des Hausärztemangels ist auch in Deutschland mittlerweile allgegenwärtig. Es dürfte nicht so gravierend sein wie zum Teil in den Medien dargestellt, doch werden bis 2020 knapp 25.000 Hausärzte ausscheiden, für die noch kein vollständiger Ersatz in Sicht ist (KOPETSCH 2010). Besonders in ländlichen, strukturschwachen Gebieten ist mit einem Versorgungsmangel zu rechnen.

Studien zum Thema Hausärzteversorgung stammen häufig aus dem außereuropäischen Ausland und arbeiten mit Hilfe von biographischen Daten und Fakten (vgl. DANIELS 2007, BOVIER und PERNEGER 2003) oder liefern lediglich Bestandsaufnahmen (vgl. KOPETSCH 2010). Die persönlichen Lebensläufe und Entscheidungen der niedergelassenen Hausärzte fanden bisweilen nur wenig bis gar keine Beachtung. Dabei können die Kenntnisse der persönlichen Motive und Entscheidungen bereits niedergelassener Hausärzte ein wichtiger Mosaikstein sein, um die Bereitschaft zur Niederlassung zu fördern. 


\section{Fragestellung und Ziel}

Die bisherigen, eher spärlichen nationalen und internationalen Forschungsbemühungen zum Thema „Hausärztemangel“ richten sich vorwiegend auf Bedarfsrechnungen und die Evaluation von Förderprogrammen. Wichtig wäre auch zu wissen - und dazu gibt es bisher meist nur Vermutungen -, was Hausärzte heutzutage zur Niederlassung bewegt beziehungsweise auf eine solche Entscheidung Einfluss nimmt. Mit Hilfe von biographischen Interviews sollen daher individuelle Gründe und Motivationen für die Entscheidung zu einer Niederlassung auf dem Land erforscht werden. Hauptziel der Arbeit ist es, den Entscheidungsprozess, der zur Niederlassung geführt hat, in der subjektiven Sicht nachzuvollziehen. Dabei liegt ein besonderes Augenmerk auf individuellen sowie familiären Einflussfaktoren bei der Niederlassung. Auch die hausärztlichen Einschätzungen der aktuellen Bemühungen zur Erhöhung der Niederlassungsrate im ländlichen Bereich können eine wertvolle Anregung für weitere gesundheitspolitische Maßnahmen sein. 


\section{Methoden}

Die Studie beruht auf der Methode der qualitativen Datenerhebung und -analyse. Das speziell hier eingesetzte Verfahren setzt voraus, dass fortwährend Material gesammelt und ausgewertet wird. So lebt das Verfahren von der Dynamik und Verzahnung der einzelnen Verfahrensschritte (Abbildung 2).

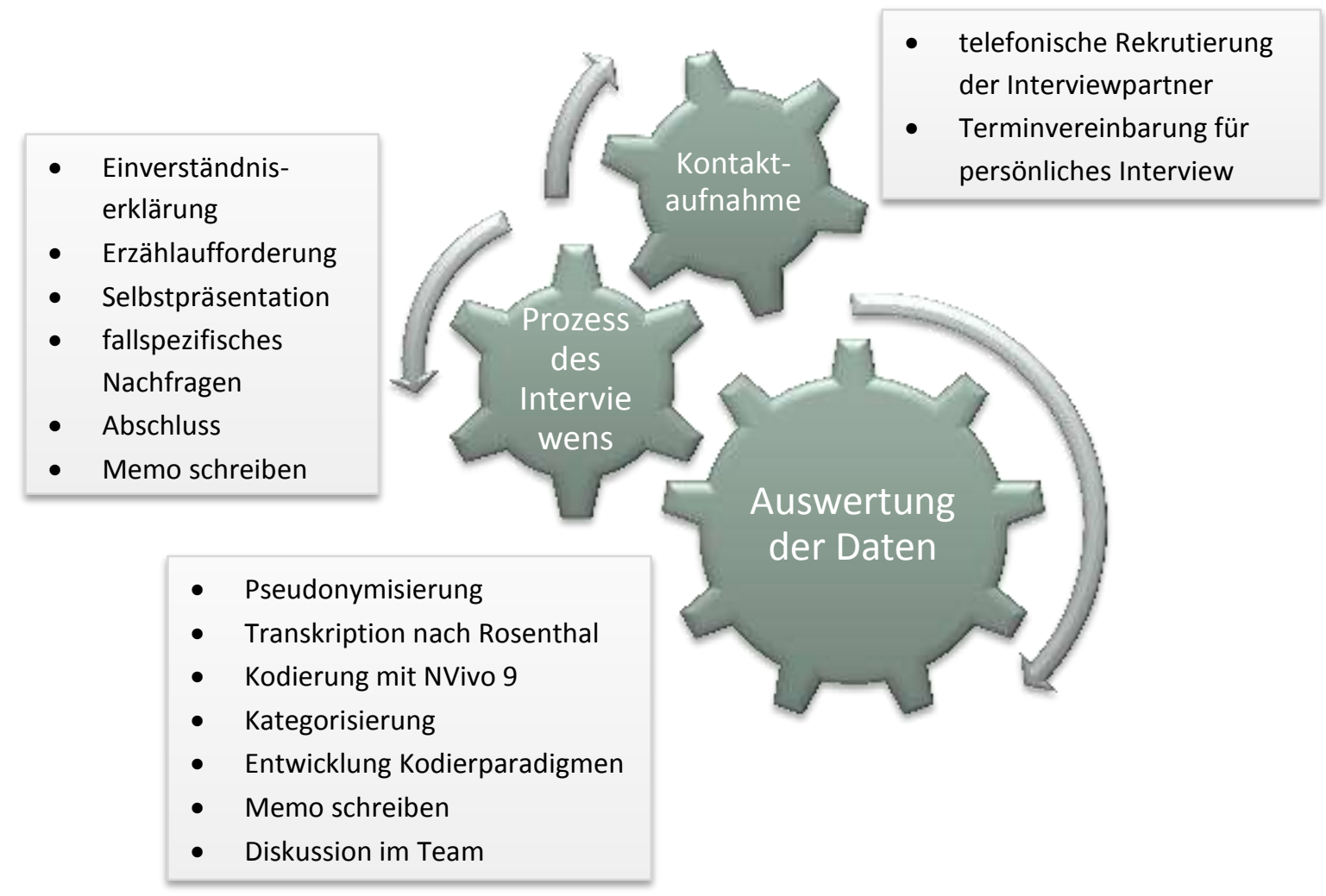

Abbildung 2 Qualitative Datenerhebung und- analyse als zirkulärer Prozess

Die Arbeit ist Teil des vom Bundesministerium für Bildung und Forschung geförderten Projekts „New practice setup of general practitioners in rural areas of Mecklenburg-Western Pomerania and Lower Saxony - a qualitative study (http://www.allgemeinmedizin.med.unigoettingen.de/de/content/forschung/359.html). Es ist ein gemeinsames Projekt der Institute für Allgemeinmedizin der Universitätsmedizin Rostock und der Universitätsmedizin Göttingen. Leiter ist Prof. Dr. Attila Altiner (Universität Rostock). Die Arbeitsgruppe in Göttingen besteht aus Prof. Dr. Wolfgang Himmel und Dr. Celia Richter. Ich selber war an allen Arbeitsschritten von der Terminvereinbarung bis zum Kodieren, ausgeschlossen der Transkription des Interviews, beteiligt. Soweit methodisch eine gemeinsame Arbeit am Interviewmaterial geboten war (beispielsweise Kodieren und Kategorisieren der Interviews; siehe Kapitel 4.3), 
war vor allem Celia Richter beteiligt; die Supervision erfolge durch Prof Dr. Wolfgang Himmel.

\subsection{Stichprobenwahl}

Für unser Studiendesign suchten wir Hausärzte, die sich in den letzten 5 Jahren im ländlichen Raum Niedersachsens niedergelassen hatten. Den ländlichen Raum definierten wir nach Größe der Städte und Dörfer. Die notwendigen Daten der Ärzte, die unsere Anforderungen erfüllten, erhielten wir von der Kassenärztlichen Vereinigung Niedersachsens. Insgesamt ließen sich 352 Ärzte in den letzten 5 Jahren in dem von uns definierten Raum nieder.

Wir wählten 100 von ihnen zufällig aus und luden sie ein, an unserer Studie teilzunehmen. Dies geschah mittels eines Informationsblattes, dass wir den Ärzten postalisch übersandten (siehe Anlage 1). 52 Ärzte bekundeten daraufhin mit Hilfe des beigefügten Antwortfaxes ihr Interesse an der Studie und ihre Bereitschaft, an einem persönlichen Interview teilzunehmen. Sie erhielten anschließend einen Fragebogen zur Erhebung soziodemographischer Angaben, den wir von 45 Ärzten ausgefüllt zurück erhielten (vgl. Anlage 2).

Auf Basis einer Kurzauswertung der Fragebögen wählten wir die ersten Interviewpartner aus. Wir hatten zu diesem Zeitpunkt lediglich eine Idee von unserer Kohorte, konnten sie aber - wie so oft bei qualitativen Forschungsfragen - noch nicht genau beschreiben, soweit es die Forschungsfrage betrifft (MERKENS 2012). So stand anfänglich für uns die Generierung von Ideen und Hypothesen im Vordergrund. Erst nach einigen Interviews ließen sich erste Rückschlüsse auf individuelle Besonderheiten und interindividuelle Parallelen finden. Von nun an griffen wir auf das theoretische Sampling zurück (siehe ausführlicher 4.3.4). Mit dessen Hilfe lassen sich Variationen und Prozesse herausarbeiten sowie auch Kategorien sättigen (STRAUSS und CORBIN 1996).

\subsection{Datenerhebung}

\subsubsection{Qualitative Interviews}

Qualitativen Interviews geben den Interviewern die Möglichkeit, „Handlungsmotive in offener Form zu erfragen“ (HOPF 2012, S.350) und somit jeder einzelnen Biographie gerecht zu werden. Sie können laut FUCHS-HEINRITZ (1984) als teilstandardisierte oder narrative Interviews geführt werden. Wir wählten das Verfahren der narrativen Interviews aus, welches vor ungefähr 40 Jahren durch SCHÜTZE (1976) entwickelt wurde. 
Um eine ganzheitliche Darstellung des Erlebten einer einzelnen Person unter Einbezug der heutigen kognitiven Fähigkeiten zu gewinnen, muss das Erlebte erzählt werden (FISCHERROSENTHAL und ROSENTHAL 1997). Der Zuhörer (Interviewer) sollte dabei nicht durch "Meinungs- und Begründungsfragen“ ins Geschehen eingreifen, da Fragen sich nur negativ auf das Entstehen eines realistischen Gesamteindruckes auswirken und den Erzähler in eine Rechtfertigungshaltung drängen (FISCHER-ROSENTHAL und ROSENTHAL 1997, S. 413). Stattdessen sollte durch eine erzählgenerierende Frage, der sogenannten „Erzählaufforderung“, das freie Erzählen gefördert werden. Die dadurch generierte „Haupterzählung“, in unserem Fall die „biographische Selbstpräsentation“, sollte nicht unterbrochen werden und wird somit allein durch den Interviewten und dessen Gedanken und Erlebten bestimmt. So können wir Rückschlüsse auf die Bedeutung und Gewichtung der Lebensereignisse für den Interviewten ziehen. Erst im folgenden Teil, dem „erzählgeneriertem Nachfragen“, ist ein Nachfragen gestattet (FISCHER-ROSENTHAL und ROSENTHAL 1997, S. 414). Durch fallspezifisches Nachfragen erreicht man ein besseres Verständnis und eine höhere Detaildichte. Auch hier sollten die Fragen offen formuliert werden. FISCHER-ROSENTHAL und ROSENTHAL (1997, S. 418) unterscheiden in diesem Zusammenhang drei Typen des narrativen Nachfragens:

1. Ansteuern einer Lebensphase: Können Sie mir über die Zeit (Kindheit, etc.) noch etwas mehr erzählen?

2. Ansteuern einer benannten Situation: Sie erwähnten vorhin die Situation (X), können Sie mir diese Situation einmal genau erzählen?

3. Ansteuern einer Belegerzählung zu einem Argument: Können Sie sich noch an eine Situation erinnern (in der Ihr Vater autoritär war; wo Sie nicht mehr an Ihren Erfolg glaubten, etc.)?

Schließlich kann dann das Interview durch „externe Nachfragen“ (FISCHER-ROSENTHAL und ROSENTHAL 1997, S. 414) zum Abschluss gebracht werden. Hier hat der Interviewer noch einmal die Gelegenheit für sich relevante Themen zu erfragen, die gegebenenfalls noch nicht zur Sprache gekommen sind.

\subsubsection{Rahmenbedingungen des Interviews}

Die erste persönliche Kontaktaufnahme mit den ausgewählten Ärzten erfolgte telefonisch, in der Regel über die angegebene Praxis-Telefonnummer. Zumeist nahmen Praxismitarbeiter den Telefonanruf entgegen, die dann entweder nach kurzer Rücksprache mit dem Arzt direkt 
einen Termin vereinbarten oder um Rückruf baten. Bei einigen Ärzten gestaltete sich die Terminabsprache als sehr schwierig, da sie bereits durch Praxis und Familie so eingespannt waren, dass für ein Interview kaum Zeit blieb. Viele Ärzte waren überrascht, dass das Interview vor Ort stattfinden würde und nicht am Telefon.

Nur zwei Ärzte verweigerten trotz der vorherigen Interessensbekundung das Interview. Alle anderen schafften Freiräume für ein ausgiebiges Gespräch. Am Telefon teilten wir vorab mit, dass die Interviewlänge erfahrungsgemäß ein bis zwei Stunden betrug, um nicht während des Interviews durch Termine des Arztes unterbrochen zu werden. Die meisten Ärzte legten sich die Interviewtermine demzufolge in ihre freien Nachmittage. Lediglich ein Arzt sagte am Morgen des Interviewtages aufgrund von Krankheit ab, vereinbarte aber einen neuen Termin. Alle andern Interviewtermine wurden wie besprochen eingehalten.

\subsubsection{Durchführung der Interviews}

Vor Beginn des Gesprächs erhielten die teilnehmenden Ärzte - nach einer kurzen persönlichen Vorstellung - einen Informationsflyer (vgl. Anlage 3), der noch einmal genau das Thema unserer Studie skizzierte und über die Datenschutzbestimmungen aufklärte. Wir gaben den Teilnehmern Zeit, den Flyer aufmerksam zu lesen und dann die beigefügte Einwilligungserklärung (vgl. Anlage 4) zu unterzeichnen. Danach begannen wir die Tonbandaufnahme mit der Erzählaufforderung:

Ich interessiere mich für Ihre Lebensgeschichte. Bitte erzählen Sie mir Ihre Lebensgeschichte, all die Erlebnisse, die Ihnen dazu einfallen. Sie können sich so viel Zeit nehmen, wie Sie möchten. Ich werde Sie auch erst einmal nicht unterbrechen, mir nur einige Notizen machen, auf die ich später zurückkomme (vgl. Anlage 5)

Wir wählten hiermit die von FISCHER-ROSENTHAL und ROSENTHAL (1997) als „offenste Form" beschriebene Aufforderung zur Berichterstattung über die eigene Lebensgeschichte. Diese sehr offene Erzählaufforderung führte bei vielen Ärzten zur Verwirrung und zur Nachfrage, ob damit nur die ärztliche Lebensgeschichte gemeint sei. Dies mochte unterschiedliche Ursachen gehabt haben. Einige schienen unsicher, vor einer durchaus fremden Person ihre Lebensgeschichte auszubreiten, andere wiederum waren das erste Mal mit einer solchen Frage konfrontiert und irritiert darüber, dass kein Fragenkatalog abgearbeitet wurde. So fielen auch die Reaktionen auf die Erzählaufforderung sehr unterschiedlich aus. Einige 
schafften es, von sich aus fast einstündige Biographie-Wiedergaben zu generieren, wohingegen andere nach nur wenigen Minuten bereits einen groben Lebensüberblick geliefert hatten und danach die Erzählung abbrachen.

Während der gesamten autobiographischen Präsentation notierten wir uns stichpunktartig Lebensaspekte, um während des erzählgenerierenden Nachfragens in chronologischer Reihenfolge auf das Wiedergegebene zurückkehren zu können. Dies schien insbesondere bei den Ärzten von Bedeutung, die nach nur wenigen Minuten bereits zum Abschluss ihrer autobiographischen Erzählung gekommen waren. Durch das chronologische erzählgenerierende Nachfragen erkannten sie "Methode“ in unserer Fragestruktur und konnten anschließend selbstständig und chronologisch in ihre Vita zurückkehren und weitere Informationen zu den einzelnen Lebensabschnitten hervorbringen. Des Weiteren fiel auf, dass aktives Zuhören von großem Nutzen war. So genügten oft freundliche Blicke oder bestätigendes Nicken, um den Erzählfluss aufrecht zu halten und immer mehr Lebensereignisse zum Vorschein zu bringen.

Das im Anschluss an die Selbstpräsentation folgende fallspezifische Nachfragen erforderte hohe Konzentration, um nicht in einen suggestiven Fragestil oder in ein Frage-AntwortSchema abzurutschen, sondern weiterhin bei einer offenen Frageform zu bleiben, die spon$\tan z u$ neuen Erzählungen führen würde. Zur Qualitätsverbesserung unserer Interviewführung wurden regelmäßig Interviews erneut analysiert.

Der Rahmen, in dem die Interviews stattfanden, war sehr unterschiedlich, meist fanden die Treffen in den Praxisräumlichkeiten statt, zuweilen jedoch auch bei den Ärzten zu Hause und einmalig in einem Restaurant. Dies hatte sicherlich auch Einfluss auf den Interviewverlauf. Im eigenen Zuhause und in den bekannten Praxisräumlichkeiten kamen den Ärzten die Worte leichter über die Lippen als in der öffentlichen Restaurantumgebung.

Die Betreuerin der Doktorarbeit, Celia Richter, führte insgesamt 10 Interviews; die Doktorandin führte 14 Interviews. Das kürzeste Interview dauerte 51 Minuten, das längste 2 Stunden 3 Minuten. Die durchschnittliche Interviewlänge betrug 83 Minuten.

\subsubsection{Transkription}

Transkription ist „die graphische Darstellung ausgewählter Verhaltensaspekte von Personen, die an einem Gespräch (z.B. einem Interview oder einer Alltagsunterhaltung) teilnehmen“ (KOWAL und O'CONNELL 2012, S. 438). Damit ist die Transkription nicht nur ein „theorie- 
neutraler Prozess", der aus den Primärdaten (Interview) über Sekundärdaten (Tonbandaufnahme) Tertiärdaten (Transkript) entstehen lässt, er nimmt auch Einfluss auf den gesamten Auswertungsprozess der Primärliteratur (KOWAL und O'CONNELL 2012, S. 440). Durch die Selektion des Transkribierenden entsteht eine Reduktion der reichhaltigen Primärdaten (COOK 1990). Um dennoch einen eventuellen Inhaltsverlust durch die Transkription so gering wie möglich zu halten, übernahmen zwei routinierte Studierende die Transkriptionen für unser Projekt. Beide arbeiteten unter Berücksichtigung verbindlicher Transkriptionsvorgaben (vgl. ROSENTHAL 2005) und nutzten die Transkriptionssoftware f4.

\subsection{Datenauswertung}

Die Auswertung erfolgte nach Methoden der Grounded Theory. Entwickelt wurde die Theorie von Barney B. Glaser und Anselm L. Strauss während einer Studie zur Interaktion mit Sterbenden in Krankenhäusern (GLASER und STRAUSS 1967). Sie wollten Theorien aus der Datenlage (grounded) heraus generieren und nicht bereits bestehende Thesen überprüfen (WOLLNY und MARX 2009). Zwar muss ein gewisses Vorwissen vorliegen, doch die eigentlichen theoretischen Konzepte sollen erst während der Analyse entdeckt und dann im weiteren Verlauf überprüft werden (HILDENBRAND 2012). So lassen sich soziale Phänomene nicht einfach nur beschreiben, sondern im fortschreitenden Prozess immer besser verstehen (WOLLNY und MARX 2009). HILDENBRAND (2012) spricht über die Grounded Theory als einen zirkulären und triadischen Prozess zur Generierung von bewährten Daten (Abbildung 3). Immer wieder muss der Forscher zu seinen Grunddaten zurückkehren, um seine Daten zu verdichten und zu bestätigen (HILDENBRAND 2012).

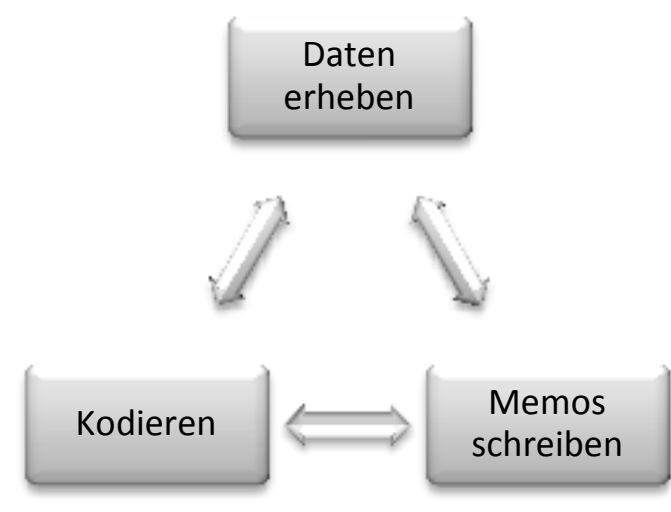

Abbildung 3 Grounded Theory als triadischer und zirkulärer Prozess (nach HILDENBRANDT 2012) Aus: Uwe Flick u.a. (Hg.), Qualitative Forschung. Ein Handbuch Copyright (C 2000 Rowohlt Taschenbuch Verlag $\mathrm{GmbH}$, Reinbek bei Hamburg 
Auch der Forscher spielt bei der Grounded Theory eine Rolle. So ist er nicht etwa neutraler Beobachter, sondern „ein beeinflussendes Subjekt im Forschungsprozess" (WOLLNY und MARX 2009, S. 470). Seine Stimmungen und Gedanken während der Datengewinnung und Analyse nehmen Einfluss auf die Datenlage und sollten daher mit reflektiert werden.

\subsubsection{Offenes Kodieren}

Der Forschungs- und Aufarbeitungsprozess beginnt damit, dass der Forscher Daten konzeptualisiert, Daten also analysiert und in ihre Einzelteile zerlegt. Laut STRAUSS und CORBIN (1996) gibt es keine Wissenschaft ohne Konzepte (Etiketten für Phänomene), weil sonst keine Fragen an die Phänomene gestellt werden können, da sie nicht genügend Aufmerksamkeit von uns erhalten. Erst wenn man das Phänomen benannt hat, kann man sich ausreichend damit beschäftigen und mit Hilfe von Fragen ihm näher kommen. So können wir beginnen zu fragen: Was ist das Phänomen? Was repräsentiert es? (STRAUSS und CORBIN 1996, S. 45).

Anfänglich geht es also darum, Konzepte zu finden, die die Daten benennen und nicht nur beschreiben. Wir benannten beispielsweise „Ich bin gerne zur Schule gegangen“ und „ich bin neugierig“ mit dem Konzept „Wissensdurst“. Diesem Begriff „Wissensdurst“ lassen sich verschiedenste weitere Ereignisse zuordnen, z.B. Buch lesen, Weiterbildungen besuchen etc.

Während der Konzeptentwicklung besteht die Möglichkeit auf Phänomene zu treffen, denen wir verschiedene Konzepte zuordnen können (STRAUSS und CORBIN 1996). Beim Lesen des Textes sollte deshalb immer die Frage „Was erfahren wir aus dieser Textstelle, um welches Phänomen geht es hier?" (WOLLNY und MARX 2009, S. 471) präsent sein. Damit sind wir beim Kategorisieren angekommen. Wir ordnen Konzepte einem Phänomen zu. So ordneten wir beispielsweise die Begriffe „Mobbing, Hierarchie und Fluktuation“ dem Phänomen „soziale Arbeitsbelastungen“ zu. Eine andere Konzeptgruppe aus „Überstunden, Dienste, Patientenzeit-Mangel“ lässt sich dem Phänomen „technische Arbeitsbelastungen“ zuordnen. Beide Gruppen können nun der Kategorie „Klinikfrust“ zugewiesen werden (Abbildung 4). Die eine Konzeptgruppe beschreibt die sozialen Faktoren, die andere die rein strukturellen (technischen) Faktoren des Klinikfrustes. Beides sind somit Subkategorien. Wie in diesem Beispiel sichtbar wird, ist der Kategorienname sehr viel abstrakter als die Konzeptbezeichnungen. Eine Subkategorie leitet also in weiterer Abstraktionsebene zur Achsenkategorie / Hauptkategorie, welches einer verfeinerten Charakterisierung des Phänomens gleichkommt. Ziel des 
gesamten Prozesses der Auswertung ist also das schrittweise Erkennen der Hauptkategorien bis hin zu einer Kernkategorie, um das Phänomen möglichst genau beschreiben zu können. Kategorien werden im Arbeitsprozess auch als „Codes“ bezeichnet.

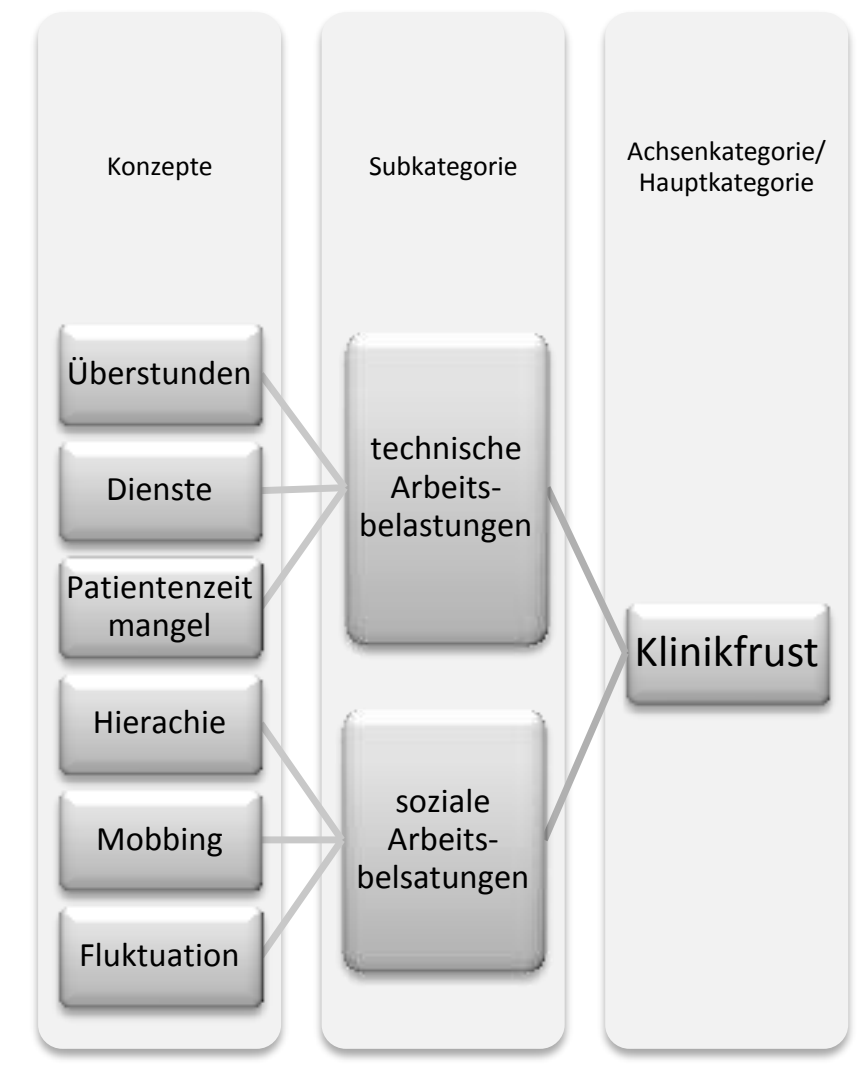

Abbildung 4 Kategorienentwicklung

Obschon eine möglichst abstrakte Bezeichnung gewählt werden sollte, steht hierbei das eigentliche Benennen der Kategorien im Vordergrund. So können auch Namen zur Benennung von Konzepten und Kategorien aus der Literatur oder gar aus der eigenen Primärliteratur, in unserem Fall also aus den Interviews, genommen werden. Letzteres wird als "In-vivo-Code“ (STRAUSS 1987, S. 33) bezeichnet. Bei uns schien z.B. der In-Vivo-Code „Mit Netz und doppeltem Boden“ (siehe 5.3.4) besonders geeignet, um das Sicherheitsbestreben unserer Ärzte zu beschreiben.

Um den gewonnenen Konzepten mehr Spezifität zu verleihen, können diese „dimensionalisiert" werden. Oft können die Bergriffe auch in ihrer dimensionalisierten Form im Text gefunden werden. Für das Konzept „Mobbing“ aus unserem Material ergibt sich z.B. diese Tabelle: 
Tabelle 1 Eigenschaften und Dimensionen

\begin{tabular}{|l|l|l|}
\hline Kategorie & Eigenschaften & $\begin{array}{l}\text { Dimensionale Ausprägung } \\
\text { (pro Ereignis) }\end{array}$ \\
\hline Mobbing & Häufigkeit & oft - nie \\
& Ausmaß & viel - wenig \\
& Intensität & hoch - niedrig \\
& Dauer & lang - kurz \\
\hline
\end{tabular}

Der eigentliche Prozess des offenen Kodierens kann sehr unterschiedlich angegangen werden: „Zeile-für-Zeile-Analyse“, ganze Sätze oder Abschnitte oder sogar das ganze Dokument auf einmal. Ersteres ist laut STRAUSS und CORBIN (1996) die ergebnisreichste Methode, die viele Konzepte entstehen lässt. Sie hilft auch besonders beim theoretischen Sampling. Wichtig für das Kodieren war zudem das Schreiben von Memos (siehe ausführlich Kapitel 4.4).

Zum Kodieren nutzten wir das Programm NVivo9. Hier lassen sich die Konzeptnamen direkt dem Text zuordnen und so immer wieder rückverfolgen (siehe Anlage 7).

\subsubsection{Axiales Kodieren}

Beim axialen Kodieren geht es darum die verschiedenen Konzepte zu verfeinern und sie im fortgeschrittenen Stadium dadurch zu den Sub- und Hauptkategorien zu verdichten (BÖHM 2012). Der Forscher springt dabei immer wieder zum offenen Kodieren und den bereits vorhanden Konzepten zurück, es wird zu einem flexiblen Prozess. Die Daten aus dem offenen Kodieren werden dabei neu zusammengesetzt und Verbindungen zwischen den Kategorien gesucht (STRAUSS und CORBIN 1996).

Letztendlich wird ein Phänomen, charakterisiert durch Kategorien, in den Mittelpunkt gestellt und ein Beziehungsgeflecht außen herum konstruiert. Hierfür lässt sich das Kodierparadigma nach Strauss nutzen (Abbildung 5). 


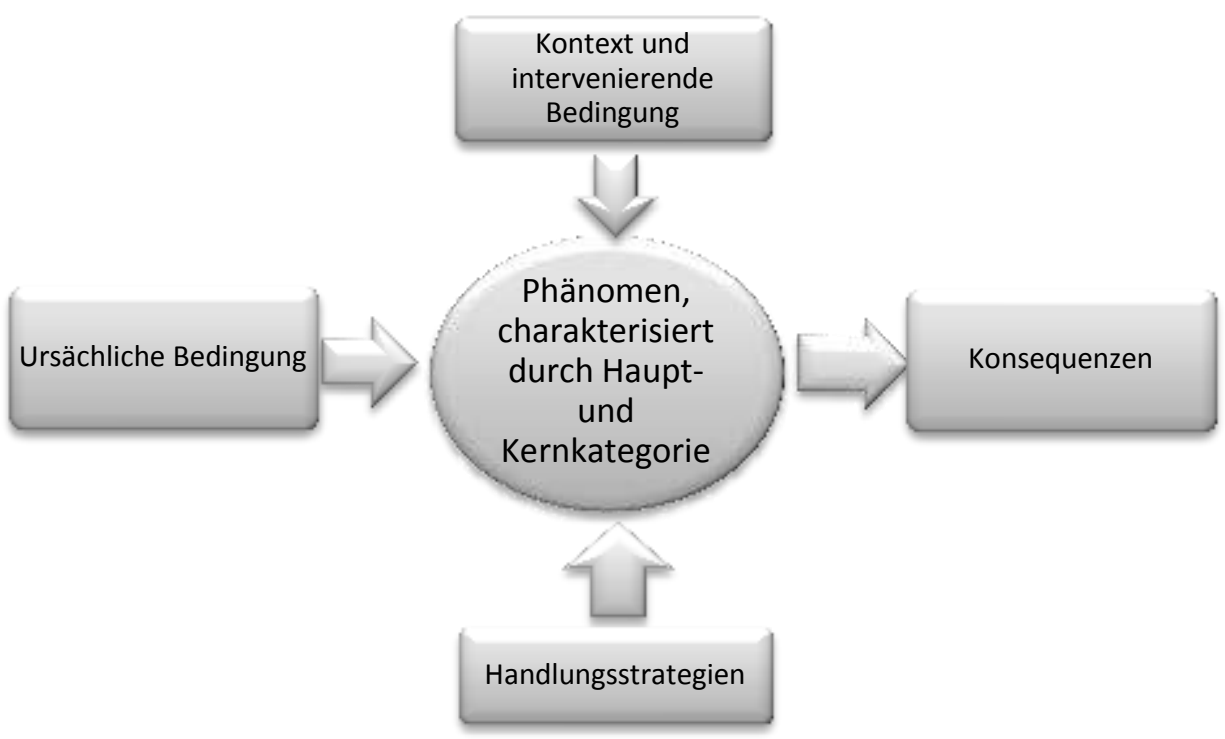

Abbildung 5 Kodierparadigma nach Strauss (Vgl. BÖHM 2012, S. 479)

Aus: Uwe Flick u.a. (Hg.), Qualitative Forschung. Ein Handbuch Copyright @ 2000 Rowohlt Taschenbuch Verlag $\mathrm{GmbH}$, Reinbek bei Hamburg

Zum Erkennen des Phänomens stellt sich die Frage „Worauf beziehen sich meine Daten? Um was drehen sich Handlungen und Interaktionen in den Daten eigentlich?“ (BÖHM 2012, S. 480).

Die „ursächliche Bedingung“ meint das grundlegende Ereignis, das einmal zum Sachverhalt des Phänomens geführt hat. Oft wird sie im Text durch Schlüsselwörter wie „weil“, „da“, „wegen“ und „aufgrund von“ angezeigt. Zeit, Dauer und Ort beschreiben den Kontext, in dem das Ereignis stattfindet. Beides ist miteinander verknüpft, da ursächliche Bedingungen nur in einem bestimmten Kontext gelten. Neben den Kontextbedingungen gibt es noch die intervenierenden Bedingungen, die ebenfalls Einfluss auf das Ereignis nehmen und zu denen das soziale, politische und kulturelle Umfeld und die individuelle Biographie der Person gehören, der das Ereignis zu Teil wird. Handlungen und Interaktionen sind zielorientierte Prozesse, die aus bestimmten Gründen durchgeführt werden, hinter denen aber keine Absicht stecken muss. Konsequenzen werden im Text häufig durch Begriffe wie "als Folge von“, „deshalb“, „mit dem Ergebnis“, „die Konsequenz war" und „folglich“ eingeläutet. Sie sind durch Handlungen und Interaktionen bedingt (BÖHM 2012, S. 481).

Wir nutzten zur Darstellung des Phänomens und den damit verbundenen Beziehungen zwischen den Konzepten und Kategorien eine etwas abgewandelte Form des Strauss'schen Kodierparadigmas. Die Beziehungsstrukturen wurden aber in gleicher Weise darstellt. So ließen sich am Ende des axialen Kodierens bereits wesentliche Konzepte graphisch darstellen und 
analytische Zusammenhänge erkennen. Beispielhaft ist die Darstellung für die Kategorie „Klinikfrust“ in Abbildung 6.

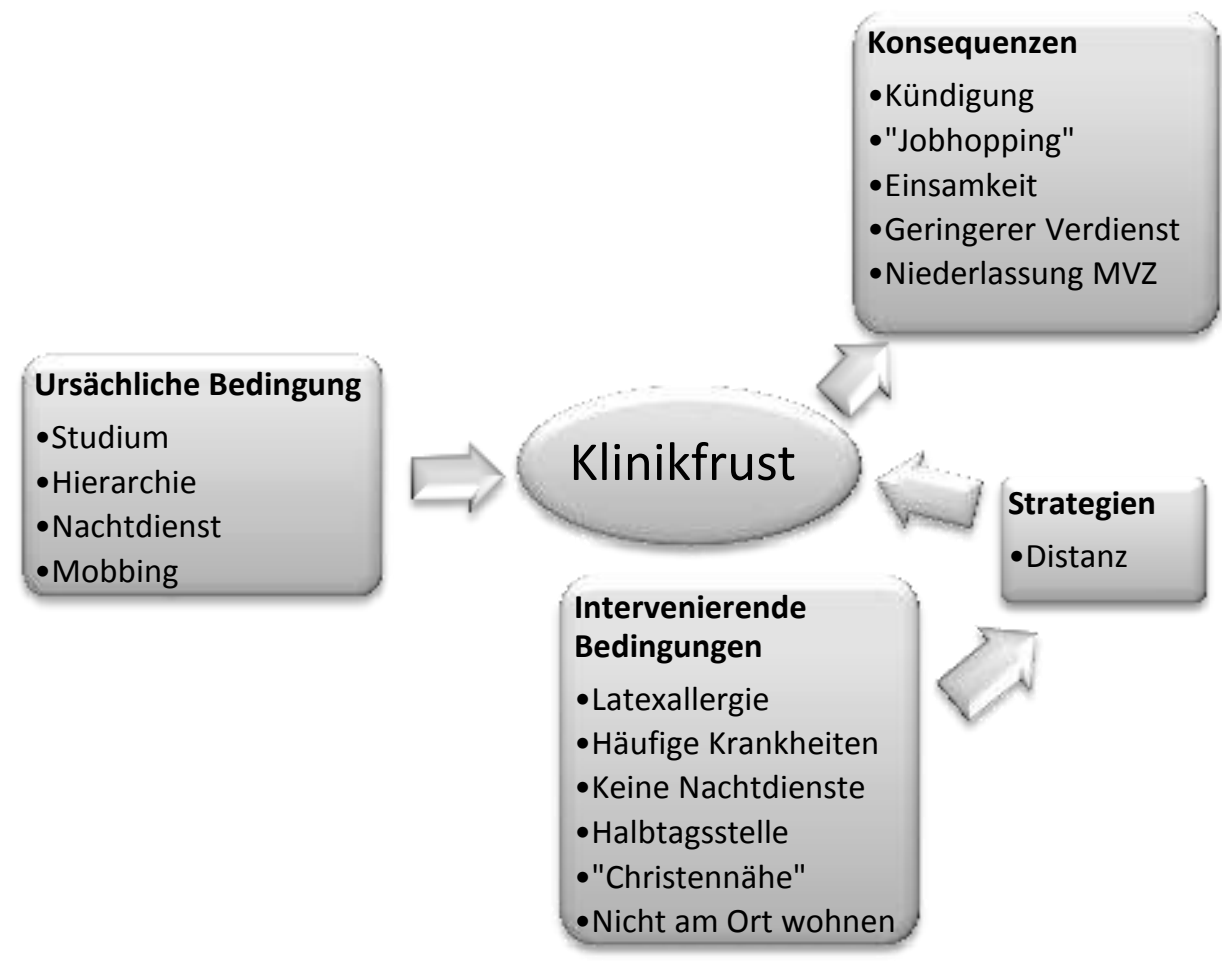

Abbildung 6 Beispiel für ein Kodierparadigma (Klinikfrust)

In diesem Zuge erstellten wir auch diverse Memos (siehe ausführlich Kapitel 4.4) zu bereits generierten Achsenkategorien, die sich in verschiedensten Interviews wiederfanden (Memo zum Thema „Klinikfrust“ im Anhang 9 als Beispiel). So konnten relevante Fakten interviewübergreifend zusammengetragen werden - in der Hoffnung, eine allgemeingültige Kernkategorie zu finden.

\subsubsection{Selektives Kodieren}

Wenn durch das vorherige Kodieren bereits mehrere gute Konzepte generiert werden konnten, kann man davon ausgehen, dass die Kernkategorie darin enthalten sein wird. Andernfalls muss auch im dritten Kodierschritt zu vorherigen Kodierschritten zurückgesprungen werden. So kann eine treffende Kategorie zu einer Kernkategorie transformiert werden oder mehrere Kategorien werden zu einer neuen Kernkategorie verschmolzen. Bei uns hob sich die Kategorie „Sicherheitsbedürfnis" als Kernkategorie ab, da sich alle vorab entwickelten Konzepte um diese Kategorie rankten. Die unter 4.3.1 dargestellte Hauptkategorie „Klinikfrust" schmälerte beispielsweise das Sicherheitsleben der Ärzte, indem es Ängste, bezogen 
auf die berufliche Zukunft, hervorrief. Zur Entdeckung der Kernkategorie ist es wichtig, das gesamte vorher erstellte Material (Memos, Codelisten, Diagramme) erneut zu sichten und einen Konsens in der Arbeitsgruppe zu finden (BÖHM 2012).

\subsubsection{Theoretisches Sampling}

Das theoretische Sampling ist eine Strategie, die die sukzessive Ziehung der Stichprobe anleitet und in engem Wechselverhältnis zum Erkenntnisfortschritt steht. Durch forschungsgeleiteten Aufbau der Stichproben sollen Konzepte gruppiert und verdichtet werden, die für die sich entwickelnde Theorie relevant sind. Die Konzepte müssen „bestätigt theoretisch relevant“ sein. „Bestätigt theoretisch relevant" bedeutet, dass Konzepte wiederholt auftauchen oder ganz abwesend sind. Ziel des theoretischen Sampling ist es, Indikatoren für Kategorien im Text zu finden und dadurch die Kategorieentwicklung voranzutreiben. Dieses Ziel steuert die Auswahl der Interviewpartner. Fragen und Vergleiche leiten das gesamte Sampling, was dazu führt, dass Variationen, Prozesse und Verdichtungen aufgespürt werden. Sampling wird durch die drei Kodierverfahren (offenes, axiales, selektives Kodieren) angeleitet. So geht es beim Sampling während des offenen Kodierens darum, so viele Kategorien wie möglich aufzudecken. Genau wie das Kodieren an sich, muss auch das Sampling in diesem Arbeitsschritt offen zu Personen, Plätzen und Situationen sein, da die Relevanz dieser noch nicht bekannt sein kann. Wichtig ist dabei, die Waage zwischen Konsistenz (relevante Datengewinnung) und weiteren Entdeckungen zu halten (STRAUSS und CORBIN 1996).

Wir haben mit einer gezielten Sampling-Technik begonnen, indem wir anfänglich Personen ausgewählt haben, die in ihren biographischen Eigenschaften sehr unterschiedlich waren. So haben wir beispielsweise dem Familienstand besondere Berücksichtigung gewidmet. Beim Sampling während des axialen Kodierens liegt der Schwerpunkt auf Beziehungen und Variationen (STRAUSS und CORBIN 1996). Während des offenen Kodierens und dem damit verknüpften Sampling entstand in unserem Fall anfänglich der Eindruck, dass besonders Ärzte mit Kindern bei der Niederlassung ein großes Bedürfnis nach Sicherheit haben. Wir schauten uns daraufhin Ärzte ohne Kinder an und stellten fest, dass sich dabei dieser Sachverhalt nicht bestätigte, auch Ärzte ohne Kinder hatten ein großes Sicherheitsbedürfnis. Ein weiteres Beispiel bezog sich in unserem Fall auf die Religiosität von Ärzten. So schien es auch hier zunächst, dass Hausärzte besonders religiös seien, doch auch dieser Eindruck konnte nicht weiter verdichtet werden. 
Der letzte Schritt, das Sampling beim selektiven Kodieren, wird auch als diskriminierendes Sampling bezeichnet. Der „Faden der Geschichte“ soll in diesem Schritt verifiziert, wenig entwickelte Kategorien aufgefüllt und bestehende Kategorien überprüft werden (STRAUSS und CORBIN 1996). Wir nutzten hierzu hauptsächlich die bereits vorhandenen Dokumente. Dieser Prozess wird so lange fortgeführt bis die inhaltliche Sättigung erreicht zu sein scheint, keine neuen Daten mehr gewonnen werden können, Kategorien ausreichend gesättigt sind und die Beziehungen zwischen den Kategorien eindeutig sind (STRAUSS und CORBIN 1996).

\subsection{Memoranda}

Das Schreiben von Memoranda, kurz Memos, stellt einen der wichtigsten Aspekte der qualitativen Forschung dar. Gedacht vor allem als Planungshilfe und Stimulans für theoretische Weiterentwicklungen, machen sie zugleich den Forschungsprozess für den Außenstehenden zugänglich sowie nachvollziehbarer und gradliniger. Aufgrund dieser Bedeutsamkeit möchte ich noch einmal gesondert auf das Verfassen von Memos eingehen, obschon in den einzelnen Forschungsschritten kurz darauf hingewiesen wurde.

Memos sind während aller Forschungsschritte zu finden. So lassen sich laut STRAUSS (1994) 8 Memotypen unterscheiden. Es gibt unter anderem erste Orientierungsmemos, die einen Überblick über das eigentliche Projekt geben sollen, so wie vorbereitende Memos, in denen die ersten Analyseteile zu Kategorien formiert werden. Hierbei können Inspirationen gefunden werden, die in einem weiteren Memo diskutiert werden. Die nachfolgenden Memos dienen dazu, aus Phänomenen Kategorien zu entwickeln und letztendlich mehrere Kategorien miteinander zu vergleichen und neue Datenerhebungen anzuregen (STRAUSS 1994).

Für uns waren die Memos für die Teamarbeit von besonderer Bedeutung. Wir verfassten sofort nach jedem Interview ein erstes Memo, das sowohl das vorher geführte Telefonat, die Atmosphäre während des Interviews als auch die inhaltlichen Aspekte festhalten sollte (vgl. Anlage 6). Dies erleichterte die Planung für weitere Interviews und erhöhte das Verständnis für die Ergebnisse des jeweilig anderen Interviewers. Auch vereinfachte es die gedankliche Rückkehr in das Interview während der weiteren Auswertungsschritte. Ideen dazu hielten wir ebenfalls in den Memos fest.

GLASER formulierte 1978 Faustregeln für das Schreiben von Memos. So hielt er die Unterbrechung des Kodierens zum Erstellen von Memos für entscheidend. Wir verfolgten diese 
Grundregel und so entstanden zu den einzelnen Interviews „Kodier-Memos“, die unsere Ideen und Entdeckungen während des Kodierens festhielten (siehe als Beispiel Anlage 8). Zudem ordneten wir den Konzepten bereits Gewichtungen zu und Phänomene kristallisierten sich leichter heraus. So zeigten sich schon in unseren Kodier-Notizen erste relevante Zwischenergebnisse. Wie schon GLASER (1978) forderte, blieben diese Memos den ganzen Forschungsprozess über offen. So konnten wir während des erneuten Sichtens der Materialen weitere Ideen hinzufügen. Zu den entwickelnden Codes erstellten wir weitere Memos, die sich während des Forschungsprozesses immer weiter verdichteten und letztendlich immer klarer und genauer wurden, bis eine Sättigung unserer Meinung nach erreicht war (als Beispiel Anlage 10). Das Memo-Schreiben erleichterte zudem maßgeblich die Teamarbeit.

\subsection{Datenschutz und Ethik}

Die Teilnahme an unserer Studie erfolgte auf freiwilliger Basis. Die Ärzte sind vorab darüber informiert worden, dass die Interviews von uns verschlüsselt transkribiert und die Tonbandaufnahmen nach der Transkription gelöscht werden. Alle Daten der Ärzte wurden pseudonymisiert und erst dann ausgewertet. Name, Anschrift, Alter, Wohnorte, Namen von Angehörigen und Freunden oder sonstige persönliche Daten (Universitätsorte, Arbeitsplätze etc.) tauchen lediglich unter einem nur von der Projektleitung rekonstruierbaren Pseudonym auf. Die Abschriften der Interviews werden nicht veröffentlicht, es werden lediglich Zitate entnommen, die nicht auf eine Person zurückzuführen sind. Eine Archivierung der Abschriften erfolgt im Institut. Die unterzeichneten Einwilligungserklärungen (Anlage 4) werden an einem sicheren Ort aufbewahrt.

Ein Ethikvotum mit der Antragsnummer 13/4/12 liegt vor. 


\section{Ergebnisse}

\subsection{Teilnahme}

Die Stichprobenauswahl erfolgte nach dem im Kapitel 4.1 erläutertem Schema. Von 100 angeschriebenen Ärzten meldeten sich 52 Ärzte positiv zurück, was einer Rückmeldungsquote von 52 \% entsprach. Alle 52 Hausärzte erhielten dann einen Fragebogen, der die biographischen Aspekte abfragte (z.B. Geschlecht, Alter usw.); 86 \% dieser Fragebögen erhielten wir zurück. Somit standen uns 45 Ärzte für ein Interview zur Verfügung, einem Rücklauf von 45 \% entsprechend. Von diesen 45 Ärzten wählten wir mit Hilfe des theoretischen Samplings 24 Ärzte zur Durchführung der Interviews aus (Abbildung 7).

352

•Hausärzte, die sich in den letzten 5 Jahren im ländlichen Niedersachsen niedergelassen haben

100

-Einladung zur Studienteilnahme (Zufallsauswahl)

52

- Fragebogen an Interessierte

45

•Fragebogen ausgefüllt

24

•Interviews (Auswahl mit Hilfe des "theoretischen Samplings")

Abbildung 7 Sample

\subsection{Beschreibung des Samples}

Insgesamt interviewten wir 12 Ärztinnen und 12 Ärzte, von denen 2/3 aus Akademikerfamilien stammten. Das Durchschnittsalter betrug 44 Jahre (Tabelle 2). Folgende Kategorien wurden gebildet, um ein differenzierteres Bild des Samples zu erhalten: Alter, soziale Herkunft, regionale Herkunft, Allgemeinmedizin als Facharztwunsch vor dem Studium, Ort in dem die Kindheit verlebt wurde (aufgewachsen), Anzahl der Kinder, Arbeit in einer Einzeloder Gemeinschaftspraxis und die Praxislage. Das Geschlechterverhältnis war in allen Kategorien ausgeglichen. Für die Definition der Praxislage übernahmen wir die Einteilungen der Projektkollegen des Instituts für Allgemeinmedizin der Universität Rostock. So ergab sich die Einteilung in Orte in ländlicher Region mit weniger als 5.000 Einwohnern, Kleinstadt mit 5.000 bis 20.000 und Mittelstadt mit 20.000 bis 100.000 Einwohner. In unserer Stichprobe 
finden sich jedoch mit einer überwiegenden Mehrheit (20 von 24 Interviewten) Niedergelassene mit Praxen in Orten bis 20.000 Einwohner und lediglich vier Orte der Kategorie „Mittelstadt" - hier allerdings mit maximal 24.000 Einwohnern.

Tabelle 2 Beschreibung des Samples (Stand 2013)

\begin{tabular}{|c|c|c|c|}
\hline & Ärztinnen & Ärzte & Gesamt \\
\hline \multicolumn{4}{|c|}{ Alter } \\
\hline $35-44$ & 6 & 6 & 12 \\
\hline $45-54$ & 5 & 5 & 10 \\
\hline $55-64$ & 1 & 1 & 2 \\
\hline \multicolumn{4}{|c|}{ Soziale Herkunft } \\
\hline Nicht-Akademikerfamilie & 3 & 5 & 8 \\
\hline Akademikerfamilie & 9 & 7 & 16 \\
\hline \multicolumn{4}{|c|}{ Regionale Herkunft } \\
\hline Niedersachsen & 9 & 7 & 16 \\
\hline restliches Bundesgebiet & 3 & 5 & 8 \\
\hline \multicolumn{4}{|c|}{ Facharztwunsch vor dem Studium } \\
\hline ja & 5 & 6 & 11 \\
\hline nein & 7 & 6 & 13 \\
\hline \multicolumn{4}{|c|}{ aufgewachsen } \\
\hline Land $(<5.000 \mathrm{EW})$ & 3 & 1 & 4 \\
\hline Kleinstadt $(5.000-20.000 \mathrm{EW})$ & 1 & 4 & 5 \\
\hline Mittelstadt (20.000-100.000 EW) & 4 & 2 & 6 \\
\hline Großstadt (>100.000 EW) & 3 & 4 & 7 \\
\hline unterschiedlich & 1 & 1 & 2 \\
\hline \multicolumn{4}{|c|}{ Anzahl der Kinder } \\
\hline keine & 4 & 4 & 8 \\
\hline 1 & 0 & 0 & 0 \\
\hline 2 & 5 & 5 & 10 \\
\hline 3 oder mehr & 3 & 3 & 6 \\
\hline \multicolumn{4}{|c|}{ Familienstand } \\
\hline ledig & 1 & 2 & 3 \\
\hline in fester Partnerschaft & 3 & 3 & 6 \\
\hline verheiratet & 8 & 7 & 15 \\
\hline \multicolumn{4}{|c|}{ Praxislage } \\
\hline Land $(<5.000 \mathrm{EW})$ & 5 & 6 & 11 \\
\hline Kleinstadt (5.000-20.000 EW) & 5 & 4 & 9 \\
\hline Mittelstadt (20.000-100.000 EW) & 2 & 2 & 4 \\
\hline Gesamt & 12 & 12 & 24 \\
\hline
\end{tabular}




\subsection{Der Weg zum Hausarzt: methodische Vorbemerkungen}

Die von den Ärzten selbst gestalteten Erzählungen wurden offen ausgewertet und im Anschluss thematisch zusammengefasst. Das Ergebniskapitel stellt die aus den Interviews hervorgegangen und hervorstechenden Themenbereiche dar. Die subjektiven Bedeutungen der Aussagewerte sollten dabei unbedingt erhalten bleiben.

In den folgenden Kapiteln werden die aus den Interviews generierten Ergebnisse beleuchtet. Zur Verdeutlichung werden aussagekräftige Zitate in den Fließtext eingebunden. Die Zitate sind durch Anführungszeichen und Kursivdruck deutlich gekennzeichnet. Füllwörter und nonverbale Äußerungen wurden - falls nicht zum Verständnis erforderlich- zur Verbesserung der Lesbarkeit aus den Zitaten entfernt. Die Ergebnisse sind mit Hilfe der qualitativen Auswertungsmethoden entwickelt worden. Zur Verdeutlichung der Wichtigkeit einzelner Aussagen werden jedoch auch quantitative Aspekte der Aussagen manchmal in Form von Zahlen, manchmal als Relationen („viele“, „wenige“, „einige“ etc.) genutzt. Diese quantitativen Aussagen beinhalten Mehrfachnennungen, da sich aus einem Interview häufig mehrere Codes generieren ließen.

\subsection{Motive für die Aufnahme eines Medizinstudiums}

Einen zentralen Stellenwert in der autobiographischen Selbstpräsentation hatte die Phase der Berufsfindung. Hier wurden besonders die Motive für das Medizinstudium erläutert. Die nachfolgende Grafik (Abbildung 8) zeigt einen Überblick über die genannten Motive, einschließlich der Häufigkeit der Motive.

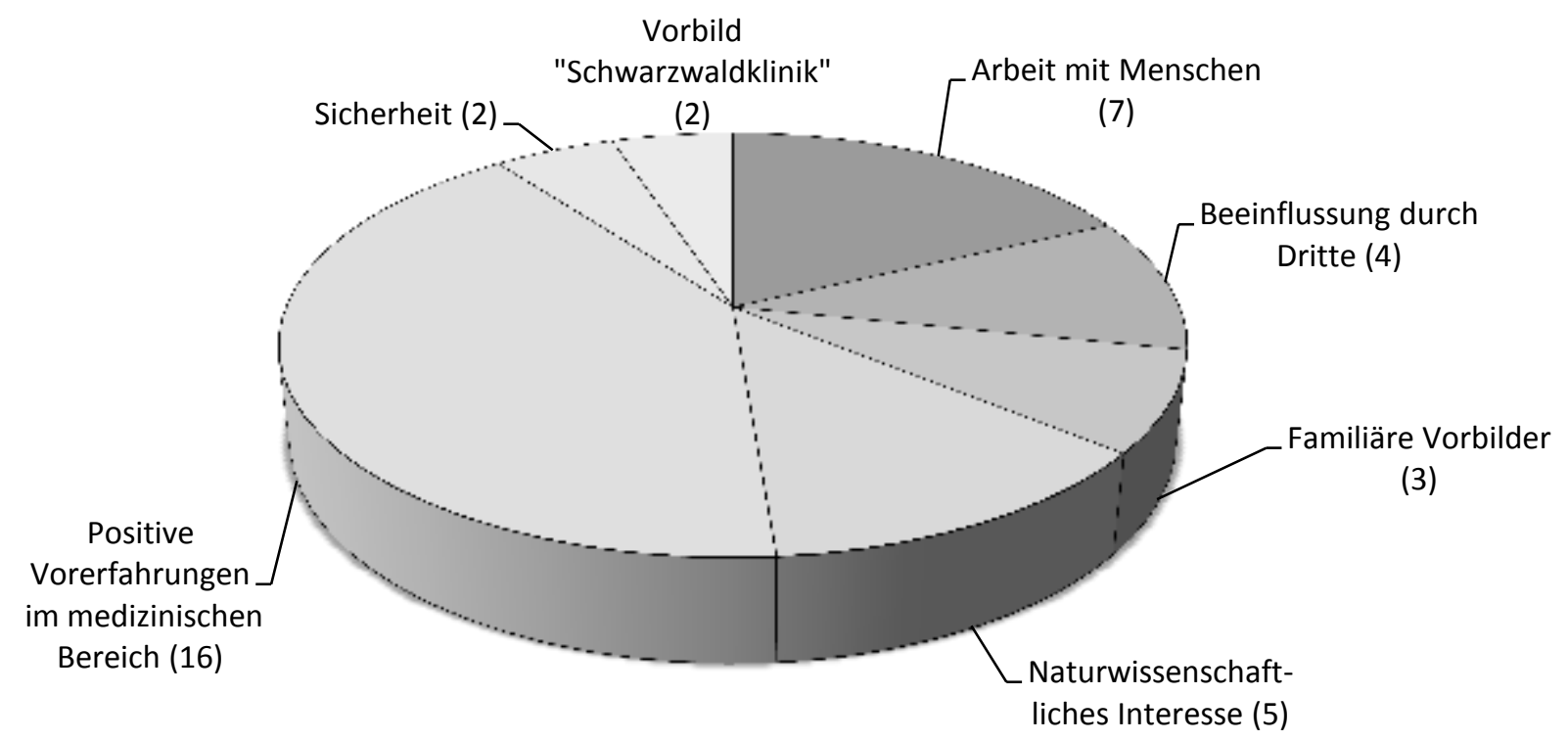




\section{Positive Vorerfahrungen im medizinischen Bereich}

In der Mehrzahl der 24 Interviews gab es Hinweise auf positive Vorerfahrungen im medizinischen Bereich. Zivildienst-/Bundeswehrerfahrungen und Ausbildungen nahmen dabei einen zentralen Stellenwert ein.

„In der Bundeswehrzeit hab ich auch schon im Sanitätsbereich mit Medizin zu tun gehabt. War damals auch schon so ein bisschen am Überlegen, ob ich nicht irgendwie in die Richtung weiter was machen sollte. " [Hausarzt, 45 Jahre]

Aber auch kurze Praktika oder die private medizinische Pflege eines Angehörigen kristallisierten sich als Einflussfaktoren auf die Studienwahlentscheidung heraus.

„Irgendwann später erkrankte meine Oma. Meine Eltern waren da längerfristig im Urlaub und dann habe ich mich quasi um sie gekümmert; ihr die ganzen Pillen sortiert und solche Dinge getan. Das war eigentlich eine gute Geschichte. Woraufhin ich dann zu dem Schluss gekommen bin, dass ich Medizin studieren wollte. " [Hausarzt, 37 Jahre]

Die positiven Erfahrungen halfen einigen Hausärzten bei der beruflichen Entscheidungsfindung, andere bestärkten sie aber auch in dem Vorhaben, Medizin zu studieren.

„Das war eigentlich vorher schon klar! Nur ich wollte wirklich wissen, ob mir das im Krankenhaus liegt. Wenn ich dann gemerkt hätte, gut die Arbeit im Krankenhaus oder das System Krankenhaus passt mir nicht, dann hätte ich mir sicherlich was anderes überlegt. Aber das hat mich einfach auch nochmal bestätigt." [Hausärztin, 43 Jahre]

\section{Arbeit mit Menschen}

Ein weiteres wichtiges Motiv im Entscheidungsfindungsprozess für ein Medizinstudium war der Wunsch, zukünftig mit und für Menschen arbeiten zu können. In 7 Interviews fand dieser Wunsch Erwähnung.

„Das fand ich total spannend. Das hat mich dann dazu gebracht, was mit Menschen zu machen und auch was Naturwissenschaftliches. Dann hatte ich die Idee, vielleicht könntest du ja Medizin machen. " [Hausärztin, 50 Jahre] 


\section{Naturwissenschaftliches Interesse}

Naturwissenschaftliches Interesse als Motiv für ein Medizinstudium konnte in etwa einem Fünftel der Interviews herausgearbeitet werden. Häufig wurde dieses Motiv zusammen mit dem Wunsch, mit Menschen arbeiten zu können, geäußert. Medizin scheint für einige unserer Ärzte eine perfekte Symbiose aus naturwissenschaftlichem und menschennahem Arbeiten zu sein. Der überwiegend naturwissenschaftlich motivierte Mediziner scheint nicht dem „Typus“ unserer Hausärzte zu entsprechen.

„ICh wollte zu dem Zeitpunkt noch nicht Medizin studieren, hab dann erst mal vier Semester Verfahrenstechnik, Chemie, Ingenieurwesen gemacht."

„Im Studium hab ich halt gemerkt, dass die Anforderungen vom Studium her nicht das sind, was ich mir so vorgestellt hab. Es war sehr theoretisch, weniger praxisorientiert. Mir hat schon der Umgang und die Arbeit mit dem Menschen gefehlt." [Hausarzt, 45 Jahre]

\section{Beeinflussung durch Dritte}

Einige der interviewten Hausärzte fühlten sich durch Dritte in ihrer Studienentscheidung beeinflusst, vor allem durch Familienangehörige. Interessanterweise befand sich unter diesen Familienmitgliedern kein Arzt, sie waren eher in medizinfernen Berufsbereichen beschäftigt.

„Meine Mutter hatte bei mir Angst vor brotloser Kunst. Sie hat mich da angemeldet und dann habe ich diesen Test mitgemacht. (...) Ich habe sehr gut abgeschnitten und konnte dann quasi gleich - einen Studienplatz bekommen. Ich habe das dann auch gemacht und habe es auch nie bereut." [Hausärztin, 46 Jahre]

Im Weiteren sprachen nur wenige Ärzte von einer Vorbildfunktion von Familienmitgliedern, die bereits im medizinischen Umfeld arbeiteten. Diese nahmen jedoch keinen direkten Einfluss auf die Berufsentscheidung der Ärzte, sondern waren leidglich ein Vorbild für sie. Der gegenteilige Effekt - ein „Anti-Vorbild“ - wird weiter unten beschrieben.

\section{Schwarzwaldklinik}

Durchaus hervorstechend war die Tatsache, dass sich etwa gleich viele Ärzte zu Vorbildern aus Film- und Fernsehen, wie zu familiären Vorbildern bekennen. Eine Ärztin nannte explizit die „Schwarzwaldklinik“ als Grund für die Aufnahme ihres Medizinstudiums. 
„Dann dachte ich mir, mach ich Medizin. Ist vielleicht doch ganz schön! Es hat mich eher die Schwarzwaldklinik geprägt, ganz ehrlich, auch, wenn's lustig klingt. Ich fand das großartig." [Hausärztin, 41 Jahre]

\section{Sicherheit}

Ein Bedürfnis nach Sicherheit fand sich in allen Lebensbereichen der Interviewten wieder. So nannten einige Hausärzte die guten Perspektiven nach dem Studium und die damit verbundene Sicherheit als einen Einflussfaktor auf ihre Entscheidung zum Medizinstudium.

„ICh hatte auch früher schon mal überlegt, ob ich vielleicht Kunst oder Biologie studiere. Bei Biologie wäre es so gewesen, dass da die Stellen natürlich relativ rar gesät sind. Da müssen Sie sich richtig hinsetzen, richtig was tun... Bei Medizin dachte ich, dass kriege ich hin." [Hausarzt, 45 Jahre]

In einigen wenigen Interviews fanden sich keine konkret benannten Beweggründe für die Aufnahme des Medizinstudiums.

\subsection{Hürden auf dem Weg zum Medizinstudium}

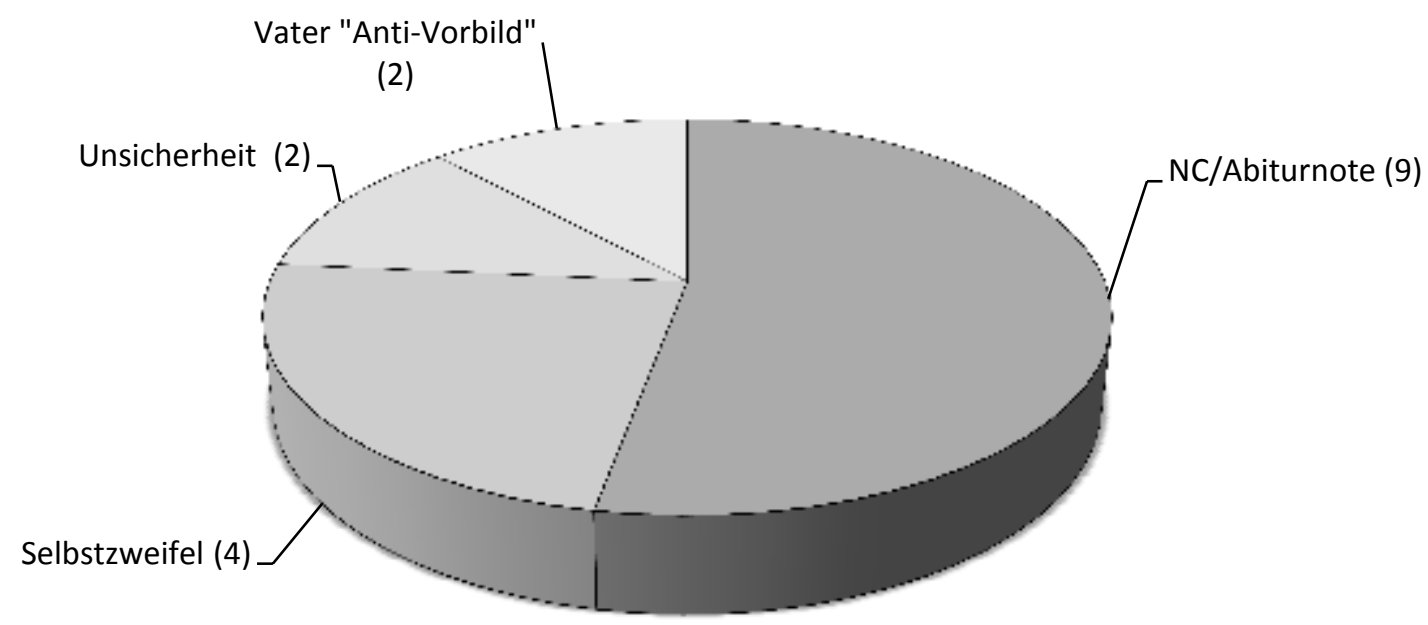

Abbildung 9 Hürden auf dem Weg zum Studium 


\section{Numerus Clausus/Abiturnote}

Der Numerus Clausus auf Medizinstudienplätze war für mehrere der interviewten Ärzte die erste Hürde auf dem Weg zum Beruf des Hausarztes. Ihre Abiturnote verhinderte den direkten Beginn des Studiums der Humanmedizin. Die Wartezeit wurde vielseitig genutzt (Ausbildung, anderes Studium etc.). Sie verunsicherte aber einige stark in der Richtigkeit ihrer Studienfachentscheidung.

„Dann auf einen Studienplatz warten. Das war eigentlich so die schwierigste Zeit. Fand ich am schwierigsten, dieses Jahr auszusetzen. Wo man auch schon -wie alt war ich damals?zweiundzwanzig war. Zu sehen, dass es nicht so richtig weiter geht, also das war im Nachhinein oder auch damals unbefriedigend. Ich hab auch so ein bisschen mit den Hufen gescharrt und irgendwie hat sich nichts getan. Man musste ja immer ein halbes Jahr warten, bis dann wieder so ein Bescheid von der ZVS kam. " [Hausarzt, 45 Jahre]

„Ich hatte schon Interesse daran, Medizin zu studieren. Aber ich glaube, diese ganzen Hürden mit dem Numerus Clausus, das war dann schon so, dass ich doch den bequemeren Weg wählte." [Hausärztin, 43 Jahre]

\section{Selbstzweifel}

Der Ruf des Medizinstudiums, besonders anspruchsvoll zu sein, ließ bei einigen Ärzten Selbstzweifel aufkommen, ob ein Studium mit einem solchen Umfang, insbesondere unter Berücksichtigung der eigenen Schulleistungen, zu schaffen sei.

„ICh hatte erhebliche Zweifel, ob's gehen würde unter diesen Voraussetzungen, aber es ging dann. Es ist keine Hetzerei gewesen, ganz normal. " [Hausarzt, 56 Jahre]

\section{Unsicherheit}

Ähnlich den Selbstzweifeln war die Hürde der Unsicherheit im Hinblick auf die Zukunft. Hier ging es jedoch um den befürchteten Mangel an externer Unterstützung und nicht so sehr um das eigene Fähigkeitspotential der zukünftigen Ärzte. Es herrschte eine Unsicherheit, ob sich das Warten auf einen Studienplatz letztendlich lohnt.

„Ich habe dann den Zivildienst als Rettungssanitäter gemacht. In dem sah ich dann, dass ich im medizinischen Bereich eigentlich weiter tätig sein wollte. Hab dann aber erst noch Sport 
studiert, weil ich keinen Medizinertest hatte und mir das auch irgendwie alles zu unsicher war. Nur weil ich jetzt nach einem Jahr Zivildienst plötzlich meinte, ok, dass könnt es jetzt wohl werden. " [Hausarzt, 40 Jahre]

\section{Vater als „Anti-Vorbild“}

Ärztlich tätige Familienmitglieder wurden etwa gleich häufig als Vorbild wie auch als „AntiVorbild“ bei der Entscheidung für das Medizinstudium genannt. Die Rolle als „Anti-Vorbild“ nahmen ausschließlich Väter ein.

„Eigentlich wollte ich nie Mediziner werden, weil mein Vater Arzt war. An gewissen Tagen hab ich ihn erst um 23 Uhr als Kind gesehen. Er hatte so eine Mondscheinpraxis, das heißt, er hat dann montags und donnerstags meistens bis dreiundzwanzig Uhr gearbeitet. Ein Hausmediziner vom alten Schlag und deshalb war für mich als Kind immer klar, dass ich nicht Arzt werde. " [Hausarzt, 38 Jahre]

\subsection{Die Entscheidung zum „Facharzt für Allgemeinmedizin“}

Voranzustellen ist der Aspekt, dass nicht alle 24 Ärzte unserer Samplegruppe eine Facharztbezeichnung in Allgemeinmedizin erworben haben. Ungefähr ein Viertel der Ärzte absolvierten die Facharztweiterbildung „Innere Medizin“ und entschieden sich erst im weiteren Verlauf für eine Niederlassung im hausärztlichen Betätigungsfeld. Die untenstehende Grafik listet die Motive, die dazu geführt haben, sich für eine hausärztliche Tätigkeit zu entscheiden, gewichtet nach negativer und positiver Motivation.

Es zeichnete sich während des Kodierens ab, dass die Entscheidung, Hausarzt zu werden, nicht ausschließlich auf positiven Motiven beruht, sondern auch negative Motive Einfluss auf die Entscheidungsfindung nehmen. Hier sind besonders negative Vorerfahrungen im Studium und Berufsleben von Bedeutung. 
Abbildung 10 Motive und Gegengründe für die Facharztwahl

\section{Klinikfrust}

„Klinikfrust“ wurde überaus häufig zum Ausdruck gebracht und zeichnete sich somit als gewichtiger Einflussfaktor ab. Das Phänomen „Klinikfrust“ zeigte in den einzelnen Interviews verschiedene Ausprägungen, z.B. „Dienstfrust“:

\section{$\underline{\text { Dienstfrust }}$}

Viele Ärzte empfanden insbesondere die vielen und langen Dienste als großes Manko für ihre Arbeit als Klinikarzt. Das Gefühl der Überforderung und die zeitliche Belastung waren dafür ausschlaggebend.

„Ich sag mal so, letztlich war in der Zeit einfach vordergründig, dass ich zum Schluss einfach auf die Dienste keine Lust mehr hatte. Muss ich ehrlich sagen, das war einfach nur noch belastend und nervend. (...) Wenn sie vierundzwanzig Stunden Dienst haben, und das zwei Mal in der Woche, die Wochenenden noch mit drin, da kommt man an die Grenzen." [Hausarzt, 45 Jahre] 


\section{Arbeitsbelastung}

Auch die hohe Arbeitsbelastung mit großem bürokratischen Aufwand und die daraus resultierende mangelnde Zeit für den Patienten stießen bei den interviewten Ärzten auf Unverständnis und führten zu einem Gefühl der Unzufriedenheit.

„Nicht von der Arbeit an sich, sondern einfach von der Arbeitsbelastung her. Das hat zum Schluss einfach keinen Spaß mehr gemacht; wenn Sie für nix mehr Zeit haben. Sie können sich für keinen Patienten Zeit nehmen, alle sind nur am Anrufen, zerren hier hin und da hin." [Hausarzt, 45 Jahre]

\section{$\underline{\text { Hierarchie/ Arbeitsklima }}$}

Nicht nur die äußeren Umstände, wie Dienstzeiten und Arbeitsbelastung, führten zur Unzufriedenheit der Ärzte während ihrer Kliniktätigkeiten. Auch die hierarchischen Strukturen und das häufig damit assoziierte und beklagte Arbeitsklima verstärkten das Unzufriedenheitsgefühl und förderten den Wunsch, Hausarzt zu werden.

„Das war mehr so eine „top-down" Mentalität. Diese sehr hierarchische Art der Leute, die schon älter sind. (...) Warum kriegt man jetzt einen über gebraten? Mir war aber immer ganz wichtig, dass ich gesagt habe: ,ich möchte meine Arztpersönlichkeit bilden und das ist unabhängig davon, was ein Oberarzt sagt oder befiehlt'." [Hausärztin, 44 Jahre]

\section{Allgemeinmedizin als zweitbeste Wahl}

Wenige Nennungen zeigten, dass die Facharztweiterbildung „Allgemeinmedizin“ auch als „Plan B“ fungieren kann. So gaben drei Ärzte an, sich für die Allgemeinmedizin entschieden zu haben, weil im gewünschten Fachbereich keine Stellen zur Verfügung standen.

„Dann habe ich mich entschlossen: Pädiatrie kriegst du jetzt keine Stelle mehr, also machst du Allgemeinmedizin. Dann kannst du immer mal ein paar Kinder mitbehandeln, dann wirfst du es nicht ganz weg. " [Hausärztin, 43 Jahre]

Letztlich zeigte sich jedoch, dass die Berufsentscheidung nicht ausschließlich darauf beruhte, den negativen Klinikfaktoren zu entrinnen, sondern, dass Punkte wie Facettenreichtum und die hohe Lebensqualität als Hausarzt die Entscheidungen ebenfalls maßgeblich beeinflusste, wie die folgenden Motive zeigen. 


\section{Facettenreichtum}

Der gewichtigste Grund für die Aufnahme der Facharztausbildung „Allgemeinmedizin“ war offensichtlich die Breite beziehungsweise Vielfalt der hausärztlichen Tätigkeit und der hohe Facettenreichtum in der täglichen Arbeit.

„So soll ein Arzt sein: zu dem gehe ich mit irgendeinem Problem, der klärt das dann oder schickt mich dann weiter. Also dieses Generelle, das man jegliches Lebensproblem mit dem Hausarzt klären kann. Das war so eine Art von Medizin, die mir gut gefallen hat." [Hausarzt, 37 Jahre]

„Was ich schätze in der Allgemeinmedizin, ist natürlich auch das Spektrum. Sie haben sowohl kleine Kinder, als auch ganz alte Patienten. Alle möglichen Erkrankungen sehen sie durch die Bank." [Hausarzt, 45 Jahre]

\section{Zeit für Patienten}

Auch die Aussicht auf einen intensiveren Patientenkontakt nahm Einfluss auf die Facharztentscheidung. So versprachen sich die Ärzte besonders durch eine hausärztliche Tätigkeit, mehr Zeit für ihre Patienten zu haben. Ebenso den Wunsch zum Aufbau langjähriger Patientenbeziehungen, mit dem Resultat der besseren Kenntnis der Patienten, hoben viele Ärzte hervor.

„Das ist auch das Schöne: die Patienten sehen wir! Ich sehe die im Prinzip von klein auf an, bis sie älter werden, bis sie erwachsen sind und vielleicht auch richtig alt werden. Das hat man natürlich in der Klinik wenig. " [Hausarzt, 45 Jahre]

Zudem wollten die Hausärzte als Vermittler an der Basis dienen und den Patienten die Medizin zugänglich machen.

„So ein bisschen an der Basis arbeiten, sicherlich auch ein bisschen die sprechende Medizin. Mach ich ganz gerne!“ [Hausarzt, 45 Jahre]

\section{Familienkompatibilität}

Als weiterer wichtiger Einflussfaktor auf die Entscheidung zur Facharztausbildung kristallisierte sich die vermutete Familienfreundlichkeit der zukünftigen Tätigkeit heraus. So entschieden sich gerade die Frauen aufgrund von besserer Vereinbarkeit von Familie und Beruf 
für die Allgemeinmedizin. Auch die Möglichkeit der Teilzeitarbeit wurde dabei positiv hervorgehoben.

„Ich wusste, wenn ich jetzt in die Klinik zurück muss, dann mache ich die Dienste. Dann muss ich irgendwie gucken, wie werde ich da eingespannt? Schwierig, auch mit Teilzeit. Also habe ich mich für die Allgemeinmedizin entschieden. " [Hausärztin, 46 Jahre]

„Das ist auch familienkompatibler! Also meine Kinder haben mir häufig gesagt: ,Es ist viel schöner, dass du jetzt häufiger zu Hause bist'." [Hausärztin, 50 Jahre]

\section{Eigener Chef}

Für einige Ärzte war auch die Aussicht auf eine Zukunft außerhalb des Angestelltenverhältnisses ausschlaggebend für die Wahl dieser Facharztausbildung. Durch eine Niederlassung als Hausarzt versprachen sie sich einen höheren Grad an beruflicher Autonomie.

„Mir ist schnell klar geworden, dass ich zwar kein Problem mit Hierarchien hab - vor allen Dingen in den Zeiten des Assistenten-Daseins nicht -, aber ich hab mich schon gefragt, wie es ist, wenn du jetzt plötzlich als relativ alter Oberarzt einen jungen Chef kriegst. Da ich dieses Erlebnis eigentlich nicht haben möchte, war mir klar, dass ich mein eigener Chef sein will." [Hausarzt, 40 Jahre]

\section{Positive Erfahrungen mit Allgemeinmedizin}

Einige Ärzte entwickelten durch positive Erfahrungen mit der Allgemeinmedizin während ihrer medizinischen Ausbildung den Wunsch, Hausarzt zu werden. Nachfolgend ein Zitat, das besonders die Relevanz des Einflusses von klinischer Lehre auf eine Facharztentscheidung hervorhebt.

„ICh hatte in der Allgemeinmedizin in [15_G_Stadt_A $]^{4}$ einen Kurs gemacht. (...) Da hatten wir eine ganz normale Vorlesung, die auch keinen interessiert hat. Also Allgemeinmedizin war total stiefmütterlich behandelt. Dann hat er einen ganz anderen Kurs mit uns gemacht (...), das war irgendwie so ein Debattierkurs. Da wurden Patientengespräche nachgespielt und mit Video aufgenommen. Wir haben uns Videoaufzeichnungen von anderen Patientengesprächen angeguckt: Was macht der falsch? Wie kommt der rein? Wie ist die Körperspra-

\footnotetext{
${ }^{4}$ Alle Ortsnamen wurden anonymisiert.
} 
che? Und so weiter. (...) Das fand ich damals schon super, dass sich überhaupt einer um das Nicht-Fachliche des Arztberufes kümmert. (...) Also das hat uns so richtig die Augen geöffnet für die ganzen "Kriegsschauplätze", die aber die Hauptsache sind. Das war großartig und spätestens da war für mich klar, dass das meine Richtung ist. "[Hausarzt, 37 Jahre]

\subsection{Motive für die Niederlassung als Hausarzt auf dem Land}

Vor der Betrachtung der Motive für die Niederlassung in ländlichen Gebieten Niedersachsens ist zu betonen, dass, wie in Kapitel 5.2 beschrieben, in unserer Betrachtung alle Städte bis 100.000 Einwohner dem „ländlichen“ Gebiet Niedersachsens (als Vorgabe) zugeordnet wurden. Während der Interviewführung fiel jedoch auf, dass gerade Interviewte in mittelgroßen Städten (in unserer Stichprobe 20000 bis 24000 Einwohner) sich zum Teil nicht dem ländlichen Gebiet zugehörig fühlten.

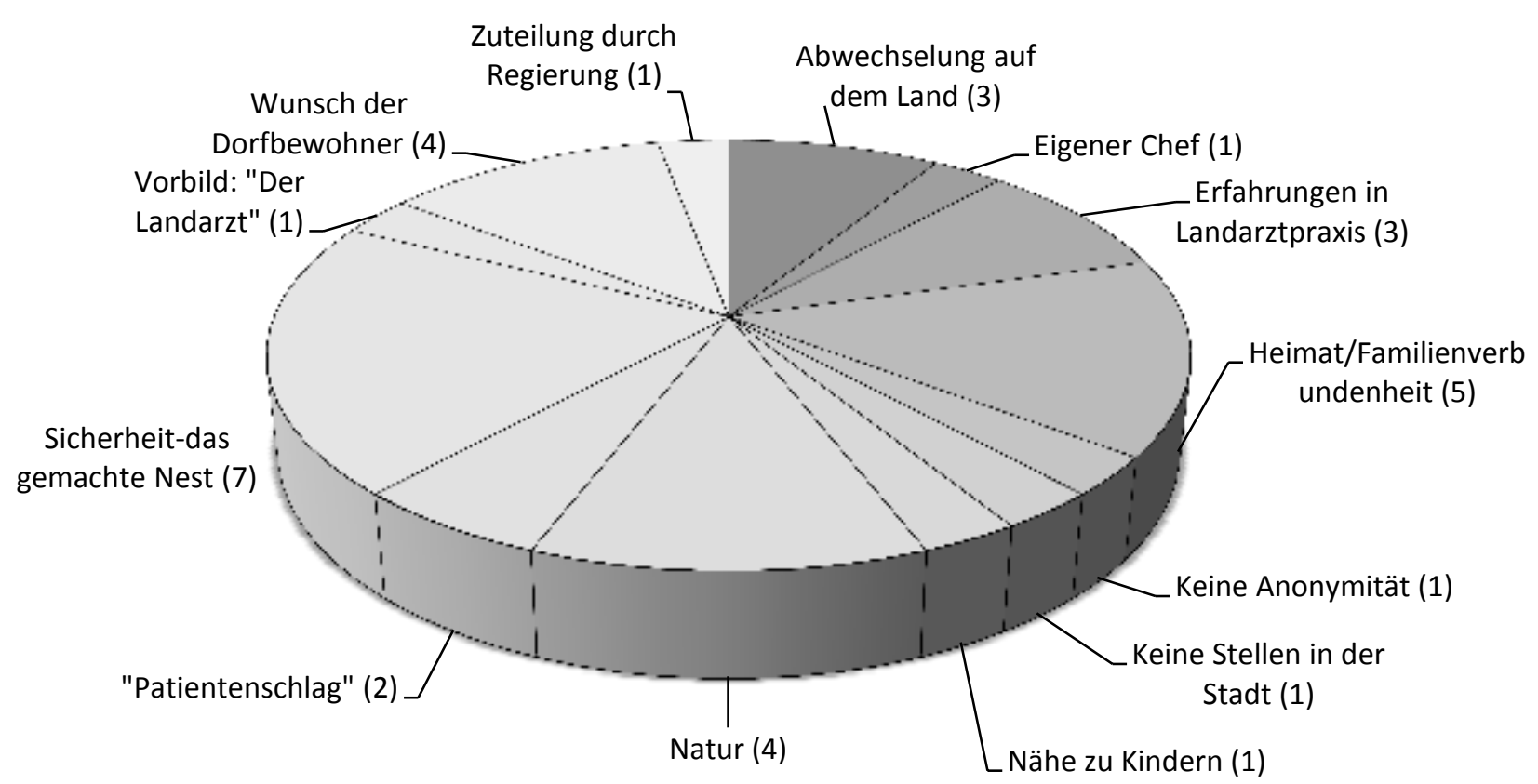

Abbildung 11 Motive für die Niederlassung auf dem Land

\section{Sicherheit - mit Netz und doppeltem Boden}

Der Sicherheitsaspekt dominierte bei der Entscheidung einer Niederlassung in ländlichen Gebieten. Gerne ließen sich die Ärzte in bereits etablierten Praxen als Teilhaber nieder oder übernahmen eine zuvor gut laufende Praxis. Die Ärzte betonten die Risikoreduktion sowohl für sich als auch für ihre Familien. Eine einfache Absicherung war für unser Ärztekollektiv nicht ausreichend, so wurde sogar „das Netz mit doppeltem Boden“ gefordert. Insgesamt 
fand sich das Bedürfnis nach Sicherheit in 7 Interviews und wurde damit am häufigsten explizit genannt.

„Also ich dachte, alleine mit drei Kindern bei null anzufangen, ist schwierig. Das ist ja ganz schwierig, mit privater Praxis neu anzufangen, deswegen die Idee, in ein bestehendes System einzusteigen. Dann zwar nicht gleich 1.000 eigene Patienten zu haben, aber die Gewähr dafür, dass das im Grunde eine Einrichtung ist, wo auch ein regelmäßiger Zulauf besteht. Sonst wäre das nicht gegangen. " [Hausarzt, 56 Jahre]

"ICh hatte aber auch hier von meinem Vermieter der Praxisräume sehr gute Mietbedingungen, keine lange Mietbindung, ich hätte jeder Zeit hier aussteigen können, mit Übernahme des Inventars. Ich hätte dann hier auch alles lassen können. Von daher hätte sich das finanzielle Desaster wirklich in Grenzen gehalten, so mit Netz und doppeltem Boden! Dann habe ich gesagt: ,Gut, dann fange ich hier an!." [Hausärztin, 43 Jahre]

\section{Heimat- und Familienverbundenheit}

Einen fast ebenso großen Einfluss hatte die familiäre und heimatnahe Bindung, verbunden jedoch meist mit dem Gedanken der Sicherheit, z.B. durch die Familienverbundeinheit am Ort. Eine frühe Verwurzelung im ländlichen Bereich scheint sich auf den ersten Blick positiv auf die Niederlassung im selbigen Gebiet auszuwirken. Interessant war jedoch, dass von den 5 Ärzten, in deren Interviews der Code „Heimat- und Familiennähe“ vergeben wurde, nur 3 aus ländlichen Gebieten stammten. Dies spricht dafür, dass eine selbstgegründete Familie und die dann erst entstehende Verwurzelung in einem ländlichen Ort ein neues Heimatgefühl wachsen lässt.

„ICh denke schon, dass das eine Rolle spielte. Also, ich bin auf dem Land aufgewachsen und ich wollte eigentlich auch wieder aufs Land zurück. (...) weil ich es einfach schöner fand, als in der Stadt. " [Hausärztin, 43 Jahre]

„Was sie halt gefreut hat, das waren die ersten Urenkel. Das war der Grund, warum wir da geblieben sind." [Hausärztin, 43 Jahre] 


\section{Erfahrungen in einer Landarztpraxis}

Unabhängig, woher die positiven Erfahrungen mit einer Landarztpraxis stammten, ob aus Film und Fernsehen oder der Realität, sie veranlassten offensichtlich junge Ärzte zur Niederlassung als Hausarzt.

„ICh weiß ehrlich gesagt nicht, wo man dieses Bild her hat. Hab ich auch schon überlegt! Also, es gab ja mal diese Fernsehserie "der Landarzt". Die hat man vielleicht ein paar Mal geguckt, aber nicht, dass es sich irgendwie reingebrannt hat, kam mehr nachher als Erklärung." [Hausarzt, 45 Jahre]

\section{Wunsch der Dorfbewohner}

Das Gefühl, gebraucht zu werden und den Dorfbewohnern zu helfen, veranlasste ebenfalls mehrere Ärzte, sich in einem ländlichen Gebiet niederzulassen.

„Hatte auch Kontakte zu Einheimischen, Bewohnern, die dann auch sagten: ,Also es herrscht eine große Unzufriedenheit hier und es war schön, wenn mal eine Alternative käme'. Auf Grund dessen habe ich dann gesagt, ich versuche, mich hier niederzulassen." [Hausärztin, 43 Jahre]

\section{Natur}

In einigen wenigen Interviews fanden sich zudem Äußerungen, die unserem Code "Natur" zugeordnet werden konnten. Besonders die Tatsache, im Grünen wohnen zu können, schien für die Ärzte attraktiv. Oft wurde dieser Begriff jedoch in einem Zuge mit „Familienzeit“ genannt. Dies wiederum lässt vermuten, dass ein Leben in der Natur besonders für Familienväter/-mütter interessant ist. Bei den Singles hingegen fanden sich oft gegenteilige Äußerungen (siehe 5.3.5.).

„Es ist halt, wie gesagt, nett im Grünen, in der Natur. Es ist einfach ein schönes Grundstück, ein schönes Haus, das gefällt mir gut." [Hausärztin, 43 Jahre] 


\section{Patienten auf dem Land}

Der „Patientenschlag“ auf dem Land kann sowohl ansprechend als auch abschreckend für eine Niederlassung als Hausarzt wirken. Hier zeigte sich sehr deutlich, dass eine Niederlassung immer mit individuellen Vorlieben verbunden ist. So konnten auch der Mangel an Anonymität auf dem Land für den einen abschreckend, für den anderen jedoch wünschenswert sein.

„Es ist sicherlich ein ganz anderer Schlag Menschen, die in der Stadt wohnen und die auf dem Lande wohnen. Ich denke, dass ich mir vorstellen kann, dass ich mit denen auf dem Lande besser klar komme als mit denen in einer Stadt. " [Hausärztin, 43 Jahre]

„Ländlich, weil wir einfach weg wollen vom Ballungsraum, von diesen Nachrichten, von diesen ganzen Schreckensmeldungen, von der Anonymität, hin zu kleinen überschaubaren Gruppen mit sozialen Anbindungen. Wir beide sind so authentisch, dass wir das auch mögen und das auch wissen. Man ist auch, in gewisser Weise, ein offenes Buch. " [Hausärztin, 44 Jahre]

Das Bild des „Landpatienten“ wirkte auf einen Teil der Ärzte abschreckend. So wurde von den Ärzten ein Patient beschrieben, der, so scheint es, ohne Sinn und Verstand zum Arzt geht und nicht der Krankheit wegen, sondern allein aus Gründen des Konsums. Der Hausarzt soll rund um die Uhr für ihn zur Verfügung stehen.

„Wir haben überwiegend ein ganz bestimmtes Klientel, wahrscheinlich ist es in Städten anders. Es ist ein Klientel mit einer häufig hohen Anspruchsrate, ohne Sinn und Verstand, wo es aus meiner Sicht rein um Konsum geht und nicht um Gesundheit. " [Hausarzt, 56 Jahre] 


\subsection{Hindernisse bei der Niederlassung auf dem Land}

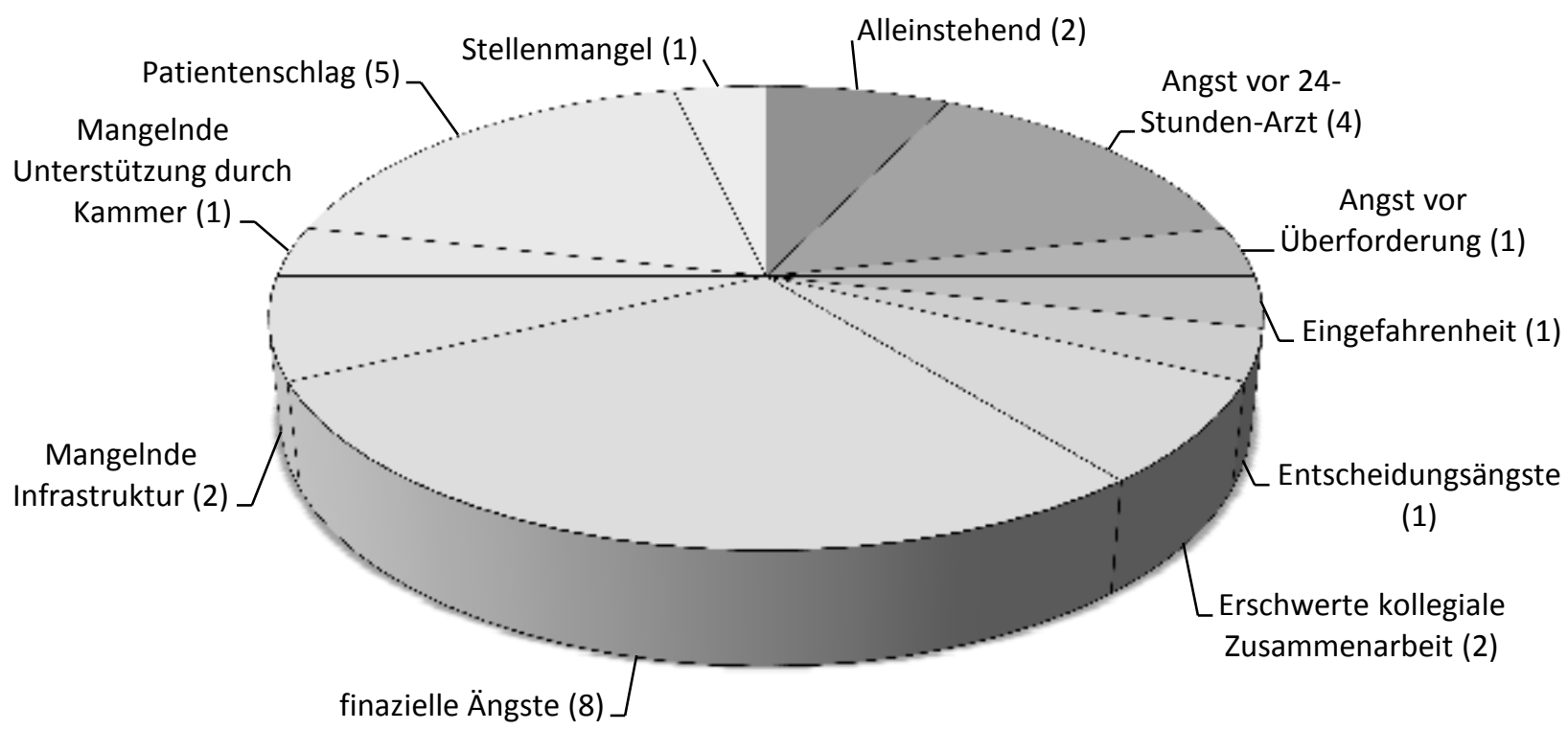

Abbildung 12 Hindernisse bei der Niederlassung auf dem Land

\section{Finanzielle Ängste}

Finanzielle Ängste beeinflussten die Niederlassungsphase am entscheidendsten. Zwar war im Fall unserer Stichprobe die Angst nicht dominant, dennoch ließen finanzielle Sorgen eine Niederlassung längere Zeit fraglich erscheinen. Berücksichtigt wurden bei der Entscheidung zur Niederlassung besonders auch die Faktoren, die das Einkommen maßgeblich beeinflussen. So informierten sich die Ärzte vorab über die Arztdichte im Umkreis, vorherige Scheinzahlen, Patientenklientel und Anzahl der Privatpatienten.

\section{„Verschiedene Faktoren ein bisschen verglichen: Verdienstmöglichkeiten, Arztdichte natür- lich, ansonsten, Patientenklientel und Privatpatienten, betriebswirtschaftlich verglichen, na- türlich. " [Hausärztin, 43 Jahre]}

Eigentum in einer strukturschwachen Region zu erwerben, war für viele der Ärzte eine unüberwindbare Hürde. Sie dachten bereits bei der Niederlassung an die Zukunft und wollten kein zu hohes finanzielles Risiko eingehen und entschieden sich für eine andere Region. 
nicht was, kauft. Dann ist es nach zwanzig Jahren nix mehr wert oder es reicht ja schon, wenn Sie es nicht mehr verkauft kriegen. " [Hausarzt, 45 Jahre]

Auch der Mangel an Eigenkapitel war besonders bei jungen Ärzten Anlass zum Nachdenken.

„Ich fühlte mich dann auch nicht so, dass ich jetzt sagte, jetzt gehst du gleich in die eigene Praxis. Das war damals recht unsicher. Man hatte bis dahin kein Geld verdient gehabt und auch kein Eigenkapital gehabt. Das war mir zu unsicher." [Hausarzt, 45 Jahre]

Die Ängste, die bei der Niederlassung eine einflussreiche Rolle spielten, wurden in unserer Stichprobe mit der Strategie gelöst, möglichst geringe finanzielle Ausgaben zu tätigen (siehe Motive für die Niederlassung: „Sicherheit - mit Netz und doppeltem Boden“). Es spiegelte sich auch hier erneut das große Sicherheitsbedürfnis wider. Große Risiken konnten und wollten die Ärzte nicht eingehen.

\section{Angst vor 24- Stunden-Arzt}

Der Mythos des „24 Stunden erreichbaren“ Hausarztes scheint noch heute selbst bei jüngeren Menschen tief verwurzelt. Die Ärzte in unserer Stichprobe stellten vor Antritt einer Stelle sicher, dass geregelte Arbeitszeiten etabliert waren, die Arbeitsbelastung zu bewältigen war und Freiraum für individuelle Freizeitgestaltung blieb.

„Also mir ist halt wichtig, dass ich auch Zeit für mich habe. Was nützt mir das ganze Geld, wenn ich es nicht ausgeben kann oder keine Zeit habe?" [Hausarzt, 45 Jahre]

„Was mir halt auch wichtig war, waren geregelte Dienste. (...) Das ist die Gefahr auf dem Lande, dass die Leute es wirklich auch immer weiter ausnutzen. In der Stadt würden die nie darauf kommen. Da haben alle Geschäfte nur um die Zeit auf und der Doktor natürlich auch. Wenn man da um 10 aufmacht, reicht es auch. Auf dem Lande ist es natürlich so, da sind dann die Ansprüche schon manchmal sehr viel anders. " [Hausärztin, 43 Jahre]

\section{Erschwerte kollegiale Zusammenarbeit}

Die jungen Ärzte schienen eine detaillierte Wunschvorstellung von ihrem idealen Arbeitsplatz zu haben. Gewisse Ansprüche sollten für sie erfüllbar sein. So waren etablierte Praxen zwar attraktiv vom finanziellen Standpunkt aus betrachtet, nicht aber die eingefahrenen 
Strukturen, die eine solche Praxis mit sich brachte. Die Ärzte wollten ihre individuellen Vorlieben in einer Praxis verwirklichen.

„Da hätte man dann auch wieder Praxisstrukturen und die Helferinnen übernehmen müssen. Ich habe auch gemerkt, so alte, eingefahren Praxisstrukturen, wie bei meinem vorherigen Chef, aufzubrechen, dass kriegen sie nicht hin. Das war auch ein Grund mit, warum ich da auch nicht geblieben bin, weil man nichts verändern kann, man ist immer der, der dazukommt. " [Hausärztin, 43 Jahre]

Auch das Verhältnis zu den Kollegen am Ort beeinflusste die Zufriedenheit in der eigenen Praxis. Ein erschwertes Arbeitsverhältnis minderte die Qualität des jeweiligen Praxisstandortes.

„Dann habe ich zwei Kollegen, für die steht im Vordergrund, ,Hoffentlich nimmt sie mir keine Patienten weg, hoffentlich arbeitet sie auch genauso lange wie ich oder genauso kurz, hoffentlich macht sie nicht zu anderen Zeiten Dienst' oder weiß ich was. Das finde ich sehr schade. Ich gucke überhaupt nicht darauf, was die anderen machen. Das interessiert mich nicht, aber mein Kollege ruft manchmal an und sagt, mein Anrufbeantworter ist nicht richtig. Dann frage ich mich, warum hört er den ab? Würde ich gar nicht auf die Idee kommen!" [Hausärztin, 43 Jahre]

\section{Mangelnde Infrastruktur}

Ein weiterer Punkt, der die Niederlassung auf dem Land für die Ärzte unattraktiv zu machen schien, war der Mangel an Infrastruktur. Hier fanden sich jedoch bei den Ärzten wieder unterschiedliche Auffassungen von „ländlich“. Ärzte im Speckgürtel großer Städte sahen sich in ihrer Vorstellung weniger als "Landärzte“.

„Gehe ich aber auf eine Praxis aufs Land, wo ich dann bin und Drumherum nichts, dann fällt Familie und Freizeit flach." [Hausärztin, 43 Jahre]

\section{Angst vor Überforderung}

Die Angst vor Überforderung bezog sich auf die Vorstellung, alleine und abgeschieden in einer Praxis auf dem Land zu sein. Kollegiale Zusammenarbeit ist daher den Ärzten wichtig. 
„Ist nicht schön, wenn man als Anfänger alleine in eine Praxis kommt. Man weiß gar nicht, wie das geht und ich finde das einfach schön auf kollegialer Ebene.( ...) Dass man einfach mal eben nachfragt, das sind eigentlich nur Kleinigkeiten, die schnell geklärt sind." [Hausarzt, 46 Jahre] 


\section{Diskussion}

Die Niederlassung als Allgemeinarzt bedeutet für die Mehrzahl der interviewten Ärzte eine Steigerung der individuellen Lebensqualität. Für sie ist das persönliche Wohlbefinden maßgeblicher Einflussfaktor in der beruflichen Entscheidungsfindung. Schlechte Erfahrungen mit den Alltagssituationen in der Klinik, gepaart mit positiven Vorerfahrungen in Hausarztpraxen, lassen diese Alternative attraktiver werden. So erhoffen sich die Ärzte durch eine Niederlassung eine geringere Arbeitsbelastung, dafür aber mehr Zeit für Patienten. Auch scheint die bessere Vereinbarkeit von Familie und Beruf von Bedeutung gerade für die jungen Ärzte. Die Familienverbundenheit ist einer der einflussreichsten Faktoren bei der Niederlassung in einer ländlichen Region. So sprechen viele Ärzte von einer innigen Heimatverbundenheit, die den Schritt einer Niederlassung auf dem Land vereinfacht. Gleichzeitig ist die Niederlassung mit einem großen Sicherheitsbedürfnis beziehungsweise finanziellen Ängsten verbunden. Bereits bestehende Praxen mit geringem finanziellem Risiko haben einen hohen Attraktivitätsgrad und wirken offensichtlich Ängsten vor finanziellem Risiko und mangelnder Unterstützung entgegen. Im Folgenden möchte ich die im vorigen Kapitel herausgearbeiteten Motive und Barrieren für einen Berufsweg hin zur hausärztlichen Niederlassung ausführlicher diskutieren.

\subsection{Ausbildungsweg}

Ein Großteil der von uns interviewten Ärzte wurde auf dem Weg zum Hausarzt durch die Abiturnote "gebremst“. Dieser Zusammenhang wird bereits durch STEINHÄUSER et al. (2013) beschrieben: Gerade die männlichen Hausärzte hatten durchschnittlich eher schlechtere Ergebnisse beim Abitur als die Kollegen, die sich eine Niederlassung auf dem Land nicht vorstellen konnten. Bei den weiblichen Kollegen traf diese Behauptung nicht zu. Unsere Untersuchung bestätigt dies und zeigt außerdem, dass die erreichten Abiturnoten das Selbstwertgefühl minimieren und die zukünftigen Hausärzte zweifeln lässt, ob ein Studium dieses Umfanges zu schaffen sei.

\subsection{Entscheidung für die Allgemeinmedizin}

Bei Betrachtung der Lebenswege der interviewten Ärzte fällt auf, dass ein frühzeitiger Kontakt mit dem Fach Allgemeinmedizin Einfluss auf das Niederlassungsverhalten nehmen kann. So verankerte sich ein positives Bild der Allgemeinmedizin, das in späteren Zeiten als Kontrast zum negativ gefärbten Klinikalltag stehen kann. Eine Untersuchung von SCHÄFER et al. 
(2009) stützt diese Erkenntnis, da die Motivation, Allgemeinarzt zu werden, nach einem Praktikum signifikant ansteigt. Dass eine Steigerung der Wertschätzung des Hausarztwesens durch frühe Kontakte erreicht wird, zeigen SCHÄFER et al. (2008) überdies in einer Studie über das Praktische Jahr in der Allgemeinmedizin. Unter Berücksichtigung dieser Ergebnisse scheinen die im In- und Ausland etablierten Verfahren zur früheren Heranführung an die Allgemeinmedizin (siehe Kapitel 2.4.1 bis 2.4.4) von Nutzen.

Viele der befragten Ärzte nutzten den Weg in die Allgemeinmedizin als Ausweg aus dem Klinikalltag. Diesen beschrieben sie als frustrierend aufgrund zu hoher Arbeitsbelastungen, zu vielen Diensten und schlechtem Arbeitsklima, bedingt durch zum Beispiel festgefahrene hierarchische Strukturen. Den Geburtenjahrgängen nach 1980 (auch als "Generation Y“ bezeichnet) ist gerade diese Hierarchie ein Dorn im Auge. So sagt man der Generation Y nach, eher den Job zu wechseln als sich anzupassen (SCHMIDT et al. 2011). Auf die schlechte Arbeitssituation in deutschen Krankenhäusern weisen ANGERER et al. (2010) hin. In dieser prospektiven Kohortenstudie wurden mit Hilfe des TAA-KH-S (Fragebogen zur Tätigkeits- und Arbeitsanalyse für Krankenhäuser) die Arbeitsbedingungen und das Befinden von jungen Klinikärzten aus München herausgearbeitet. Es zeigte sich ein Mangel an Ressourcen wie „Tätigkeitsspielraum“ und „Partizipation“ bei gleichzeitig hoher Belastung durch Zeitdruck und Personalmangel. Des Weiteren stieg der Anteil von Mobbingopfern während des dreijährigen Beobachtungszeitraums auf $15 \%$ an. In einer Untersuchung zur beruflichen Situation junger Ärztinnen und Ärzte (OBERLANDER 2010) geben lediglich 44 \% der Krankenhausärzte an, mit ihrem Beruf sehr zufrieden/zufrieden zu sein, 14,8\% sind sehr unzufrieden/unzufrieden. Bei den niedergelassenen Ärzten hingegen sind deutlich mehr Ärzte $(68,1$ \%) sehr zufrieden/zufrieden und nur $4 \%$ sehr unzufrieden/unzufrieden.

Führen also die positiven Kontakte mit der Allgemeinmedizin im Vorfeld oder aber die widrigen Krankenhausbedingungen letztendlich zu einer Niederlassung? In unserer Untersuchung überwiegen die positiven Erfahrungen mit der Allgemeinmedizin. Im Vergleich zur Studie von ANGERER (2010) konnten wir feststellen, dass besonders die besseren zeitlichen und familiären Ressourcen sowie der Abbau von Stressoren die Allgemeinmedizin für Ärzte attraktiv macht. Auf Seiten der Ressourcen sind auch der Tätigkeitspielraum und erhöhte Partizipationsmöglichkeiten durch die Niederlassung und die damit verbundene Selbständigkeit hervorzuheben. Stressoren wie personelle Fluktuationen, Zeitdruck durch Termine und Ar- 
beitsmenge und widersprüchliche Aufträge, die zuvor am Klinikalltag bemängelt wurden, konnten abgebaut werden.

Auch der Reichtum an Facetten in der Allgemeinmedizin ist in unserer Untersuchung als gewichtiger Faktor in den Vordergrund getreten. Das breite medizinische Spektrum und das damit verbundene groß gefächerte Tätigkeitsfeld führten bei unserer Stichprobe zu der Entscheidung einer hausärztlichen Niederlassung. Dies steht im Kontrast zu den Ergebnissen einer 2008 veröffentlichten Studie aus Bremen (NIEHUS et al. 2008), wonach trotz positiver Erfahrungen durch Praktika in der Allgemeinmedizin bei den Medizinstudierenden die Allgemeinmedizin nach wie vor als „langweilig“ eingestuft wird und „die bereits bestehenden Spezialisierungswünsche“ nicht mehr geändert werden. Es könnte sein, dass später die negativen klinischen Erfahrungen Einfluss nehmen und den anfänglichen „Glanz“ der spezialisierten Fächer schmälern. Es scheint, als würde die Symbiose aus negativen klinischen Erfahrungen und positiven Vorerfahrungen mit der Allgemeinmedizin zu einer vermehrten Niederlassung führen.

Die Hausärzte entsprechen deutlich ihrer Generation und handeln auch dementsprechend. Dies beeinflusst somit auch ihre Professionsfindung. So haben sie große Ansprüche an den Arbeitsplatz. Sie wünschen Abwechslung im Tätigkeitsfeld, individuelle Entscheidungsfreiheit und Teamarbeit statt Hierarchie (SCHMIDT et al. 2010). Die Allgemeinmedizin kann der nachwachsenden „Generation Y“ somit viel bieten.

Die bessere Vereinbarkeit von Familie und Beruf, die laut unserem Ärztekollektiv ebenfalls an der Facharztentscheidung Beteiligung fand, scheint besonders unter Berücksichtigung der Aussage von KOPETSCH (2010, siehe Kapitel 2.1), dass der Großteil des Ärztinnenschwundes auf die schlechte Vereinbarkeit von Familie und Beruf zurückzuführen sei, von besonderer Bedeutung. Dieses Bild zeigt sich auch in einer Internetumfrage unter Medizinstudierenden des Deutschen Ärzteblattes (RICHTER-KUHLMANN 2007). So glaubten $74 \%$ der befragten Studierenden, dass eine bessere Vereinbarkeit in einer Praxis möglich ist und nur $14 \%$ glaubten an die bessere Vereinbarkeit im Krankenhaus. Spekulieren lässt sich an dieser Stelle über die Sinnhaftigkeit der Förderung dieses positiven Images, um dem Problem des Hausärztemangels entgegenzuwirken. 


\subsection{Niederlassung im ländlichen Raum}

Bei der Niederlassung in ländlichen Gebieten dominiert der Sicherheitsaspekt. Er kann die Niederlassungsbereitschaft sowohl fördern als auch verringern. So sind bereits etablierte Praxen mit geringem finanziellem Risiko besonders attraktiv für eine Niederlassung auf dem Land. Die Gefahr, sich in einer ländlichen Region eine Immobilie zuzulegen, ohne zu wissen, wie sich die Region entwickeln wird, schien hingegen die Teilnehmer unserer Stichprobe potentiell abzuschrecken. Generell ist die finanzielle Angst der wichtigste Faktor, der gegen eine Niederlassung spricht. Auch in einer Studie aus den USA erhöhte sich die Wahrscheinlichkeit einer Niederlassung auf dem Land bei finanziellen Anreizen (BÄRNIGHAUSEN und BLOOM 2009). In unserem Fall scheint es bereits auszureichen, wenn finanzielle Sorgen gemindert werden.

Die Familie spielt auch bei der Niederlassung in ländlichen Bereichen eine besondere Rolle. So ist unser Code „Heimat- und Familienverbundenheit" am zweithäufigsten vertreten bei den Gründen für eine Niederlassung auf dem Land. Dieses Bild zeigt sich auch im internationalen Vergleich. DANIELS et al. (2007) zeigten, dass der familiäre Ursprung in einer ländlichen Region sowohl für die erste Niederlassung im ländlichen Bereich als auch für die langfristige Niederlassung eine signifikante Rolle spielt. Zudem postulieren sie, dass es sinnvoll sei, Förderprogramme speziell für Medizinstudierende aus ländlichen Bereichen zu entwickeln. Dies scheint auch angesichts unserer Ergebnisse sinnvoll.

Die Familie ist mit der Freizeit eng verzahnt, Freizeit ist oft Familienzeit. Aus diesem Grund fürchten die Ärzte, bei der Niederlassung auf dem Land alte Strukturen und Gewohnheiten übernehmen zu müssen. Besonders der 24 Stunden erreichbare Arzt flößt ihnen Angst ein. Auch hierfür findet man in Studien zur Genrationsentwicklung (s.o.) eine Begründung. So wollen die Nachfolger der „Babyboomer“-Generation nicht länger Arbeitszeiten absitzen und fordern eine gute Begründung für Überstunden. Die neue Generation Hausärzte unterscheidet sich somit maßgeblich von den vorherigen Hausärzten der „Babyboomer“-Generation, die noch „lebten, um zu arbeiten“ (SCHMIDT et al. 2011). Dieses Wissen um die Generationen und ihre Veränderungen bei Einstellungen und Lebensweisen scheint von entscheidender Bedeutung.

Aber auch die individuellen Bedingungen, die eine Praxis mit sich bringt, sind entscheidend für die jungen Ärzte. Erschwerte kollegiale Verhältnisse am Ort sowie eingefahrene Struktu- 
ren der Praxis machen die Arbeit unattraktiv. Die jungen Ärzte wollen Entfaltungsmöglichkeiten und eigene Kompetenzen. Sie „leben bei der Arbeit" und haben so ein hohes Anforderungsprofil an ihren eigenen Arbeitsplatz. Hinzu kommt, dass die Jüngeren die kollegiale Zusammenarbeit immer größer schreiben (SCHMIDT et al. 2011). So fürchten die Ärzte bei einer Niederlassung in ländlichen Gebieten, sofern keine Gemeinschaftspraxis vorhanden ist, einen Mangel an ausreichendem kollegialem Kontakt - sei es durch die mangelnde Infrastruktur oder durch die Entfernung zu anderen Kollegen.

In einer Studie von DANIELS et al. (2007) fanden sich Hinweise darauf, dass der starke und öffentlich geäußerte Wunsch einer Gemeinde nach Ärzten ebenfalls das Niederlassungsverhalten beeinflusst. Dies kam in unserer Studie nicht so eindrücklich zum Ausdruck. Lediglich der Code „Wunsch der Dorfbewohner" entsprach dem Ergebnis der Studie aus New Mexico/USA. Ob die deutschen Ärzte ihre persönlichen Bedürfnisse an denen der Bevölkerung ausrichten, bleibt fraglich.

Oft in Kombination mit der oben angesprochenen „Familienzeit" verwendeten wir auch den Code „Natur“. Die Natur hat einen hohen Wert für das individuelle Wohlbefinden. Sie wird häufig als Grundbedürfnis betitelt (LOUV 2011). Laut REEH (2008) sei die Sehnsucht nach Natur eine Reaktion auf die naturfernen Lebensbedingungen der heutigen Zeit. An dieser Stelle lässt sich spekulieren, ob der medizinische Alltag in Krankenhäusern solche naturfernen Lebensbedingungen für die Ärzte verkörperte. Wahrscheinlicher ist jedoch, dass ein Naturbedürfnis auch mit dem Bedürfnis nach einer Steigerung der Lebensqualität einhergeht, da in unserer heutigen Gesellschaft die Natur mit positiven Bedeutungen assoziiert ist ( $\mathrm{KIRCH}-$ HOFF 2012).

\subsection{Stärken und Schwächen der Untersuchung}

In den qualitativen Interviews konnten die Ärzte die Themen gemäß ihrer subjektiven Relevanz gestalten. Dies führte allerdings dazu, dass in einigen Fällen die individuelle Terminplanung eine Verkürzung des Interviews erzwang. Zumeist konnte aber der Interviewleitfaden flexibel angepasst werden, so dass auch unter Termindruck ausreichendes Informationsmaterial gewonnen wurde, insbesondere, da die zu Beginn der Interviews erfolgten freien autobiographischen Erzählungen bei allen Interviews sehr ausführlich ausfielen. 
Bei einigen wenigen Interviews ist anzunehmen, dass aufgrund der vorab versandten Informationen über das Forschungsthema (siehe Anlage 1) eine überdeutliche Darstellung der eigenen Positionen zu den aktuellen politischen Bemühungen zum Hausarztmangel zum Ausdruck gebracht wurde. Ich versuchte, dies bei der Analyse zu berücksichtigen. Zudem ist anzunehmen, dass aufgrund der Frage nach der ganz persönlichen Lebensgeschichte und nicht nur nach dem beruflichen Werdegang emotional zu tangierende oder unangenehme Lebensereignisse bewusst zurückgehalten wurden, obschon einige sehr emotionale Interviews stattfanden. So entstanden bei der Analyse zum Teil Schwierigkeiten bei der Nachvollziehbarkeit der Lebenswege.

Das Setting der einzelnen Interview-Orte unterschied sich sehr voneinander (siehe Kapitel 4.2.3). Dies führte zu sehr verschiedenen Interviewsituationen. So gab es in den Praxisräumlichkeiten vermehrt Unterbrechungen durch Telefonate oder Absprachen mit dem Praxispersonal, in den privaten Räumlichkeiten durch weitere Familienmitglieder oder aber während des Restaurantbesuches eine erhöhte Geräuschkulisse. Bei dem Interview im Restaurant kam erschwerend hinzu, dass eine Interviewunterbrechung zum Essen stattfinden musste, sowie die Hemmschwelle, persönliche Dinge preiszugeben in Gegenwart sämtlicher anderer Gäste erhöht war.

Nicht nur die Fragenauswahl nimmt Einfluss auf die Ergebnisse, sondern auch die Auswahl der Interviewpartner. So nimmt bereits die Zugänglichkeit zum Sample Einfluss auf die spätere Untersuchungsgruppe (BURGESS 1991). Mit MORSE (1994) könnte man in unserem Fall von einer sogenannten sekundären Selektion sprechen, da wir auf eine Rückmeldung der Ärzte angewiesen waren. Erst nachdem die ersten Ärzte ihre Teilnahme zugesichert hatten, konnte ein Sample kreiert werden, dass die größtmögliche Varianz bezogen auf die Charakteristika der Interviewpartner bot.

Es muss davon ausgegangen werden, dass - obwohl auch eine geringe Vergütung in Aussicht stand - sich ausschließlich Ärzte der Befragung zur Verfügung stellten, die grundsätzlich offen gegenüber Forschungsanliegen und Verbesserungsmöglichkeiten sind. Zwar konnten zum Teil Ablehnungsgründe erfragt werden, dennoch lässt sich über die genauen Gründe nur spekulieren. Des Weiteren ist festzuhalten, dass wir ausschließlich aktuell noch niedergelassene Ärzte befragt haben, bei denen eine Niederlassung erfolgreich gelungen ist. Daten über 
Ärzte, die vergeblich versucht haben, sich niederzulassen oder die die Niederlassung wieder aufgegeben hatten, konnten nicht berücksichtigt werden.

Dem Sample liegt keine statistisch repräsentative Kohorte zugrunde, sondern es sollte laut MERKENS (2012) versucht werden, ein möglichst umfassendes Bild der Grundgesamtheit zu konstruieren. So beinhaltet unser Sample bewusst möglichst kontrastreiche Fälle, die sich aus unseren Auswertungen der einzelnen Interviews ergaben und demnach durch uns beeinflusst wurden.

Die hier verwendete Definition von „ländlich“ ist pragmatisch gewählt. Eine genauere Betrachtung der Ortsfaktoren anhand verschiedener Parameter (z.B. öffentliche Verkehrsmittel, Einkaufsmöglichkeiten etc.) wäre sinnvoll, bezogen auf die Frage, wie ländlich eigentlich „ländlich“ ist. So ließen sich Standorte genauer beschreiben und ihr „Ländlichkeitsfaktor" nicht nur anhand von Einwohnerzahlen festmachen.

Zwar sollte es bei der qualitativen Forschung nicht darum gehen, theoretische Vorkenntnisse zu überprüfen, dennoch ist die Kenntnis darüber unverzichtbar (HILDENBRAND 2012). Auch wenn wir versuchten, die größtmögliche Offenheit gegenüber neuer Forschungserkenntnisse mitzubringen, so kann man sich nicht davon freimachen, bei der qualitativen Analyse sein persönliches Vorwissen mit zu berücksichtigen. Auch spielt die subjektive Wahrnehmung des Forschers im Feld eine Rolle bei der Produktion der Daten, dies beeinflusst die Datenfindung (WOLLNY und MARX 2009, S. 470). Obwohl wir versucht haben, den höchstmöglichen Grad an Nachvollziehbarkeit durch die ausgiebige Dokumentation zu erzielen, wird dennoch immer ein persönlicher Einfluss nachzuweisen sein.

\subsection{Schlussfolgerung und Ausblick}

Die Interviews mit den Hausärzten zeigten, dass der Weg zum Hausarzt beschwerlich sein kann. Sowohl in der Ausbildung als auch bei der Niederlassung können die Bedingungen der Hausärzte verbessert werden. Anhand der vorliegenden Studienergebnisse lassen sich Vorschläge zur Verbesserung der Hausarzt-Politik entwickeln.

\section{Zulassungsbedingungen zum Medizinstudium}

Sowohl in unserer Studie als auch in vorherigen Studien (beispielhaft STEINHÄUSER et al. 2013) stellte sich der Numerus Clausus zur Zulassungsbeschränkung des Medizinstudiums als Problem für die zukünftigen Hausärzte dar. Alternative Zulassungsmöglichkeiten könnten 
helfen, dass mehr potentielle Hausärzte den Weg in das Medizinstudium finden. Der ehemalige Gesundheitsminister Rösler wollte bereits 2010 eine „Medizinerquote“ 5 (MIHM 2010) einführen, die nun auch der neue Gesundheitsminister Gröhe fordert. Dies scheint angesichts unserer Studienergebnisse ein sinnvoller Ansatz, der weiter verfolgt werden sollte.

\section{Frühes Heranführen an die Allgemeinmedizin}

Wichtig ist es zudem, zukünftigen Hausärzten früh die Möglichkeit zu geben, die Allgemeinmedizin für sich zu entdecken. Sie könnten erfahren, welche Alternativen es zu den klinischen Fachrichtungen gibt und welche Vorzüge die Allgemeinmedizin mit sich bringen kann. Die bereits gestarteten Modelle, wie beispielsweise „Klasse Allgemeinmedizin“ (STEGER et al. 2012), sind ein guter, aber noch ausbaufähiger Ansatz. Ob solche Projekte wie diese verpflichtend für alle und nicht nur für die ohnehin schon interessierten Studierenden einzuführen wären, ist zumindest überlegenswert. Wichtig ist dabei, möglichst früh in den klinischen Studienabschnitten solche Projekte zu verankern. So könnte sich die Allgemeinmedizin als gleichwertiges Fach neben den „großen klinischen Fächern“ wie Innere Medizin und Chirurgie etablieren.

\section{Finanzielle Sicherheit}

Der größte Teil der Ärzte, die sich als Hausärzte niederlassen, steigert dadurch ihre persönliche Lebensqualität. Indem sie der Tätigkeit im Krankenhaus „entfliehen“, erfahren sie einen Zugewinn an Selbstbestimmung; auch Familie und Beruf sind besser miteinander zu vereinbaren. Allerdings drohen die mit der Niederlassung verbundenen potentiellen finanziellen Sorgen die Lebensqualität zu schmälern. So gehen die Hausärzte von morgen nur ungern finanzielle Risiken ein, sondern bevorzugen eine Niederlassung mit „Netz und doppeltem Boden“. Gern werden bereits etablierte und vollausgestattete Praxen übernommen. Auch finanzielle Unterstützungen erleichtern in einigen Fällen die neue Selbstständigkeit.

Aus diesen Gründen sind finanzielle Programme zur Unterstützung der Niederlassung unbedingt zu befürworten (siehe 2.4.3.). Durch Programme dieser Art könnte das Startkapital der Ärzte erhöht werden und eine Niederlassungsbewegung, auch auf dem Land, positiv beein-

\footnotetext{
${ }^{5}$ Medizinstudenten, die bereit sind, sich nach dem Studium auf dem Land niederzulassen, sollen zukünftig bei der Studienplatzvergabe bevorzugt werden.
} 
flusst werden. Ideen wie „Eigenpraxen ${ }^{\prime 6}$ könnten ebenso Berücksichtigung finden (HAFFKE 2013), da die Ärzte hier kein eigenes finanzielles Risiko haben. Fraglich ist jedoch, ob dieses Anstellungsverhältnis die Selbstverwirklichungstendenzen der nächsten Generationen ausreichend befriedigt (SCHMIDT et al. 2011).

Um weitere Probleme bei der Niederlassung zu identifizieren, wäre eine vergleichbare Studie über Ärzte, die ihre Niederlassung aufgegeben haben, wünschenswert. Ergebnisse darüber, welche Aspekte letztendlich zum Aufgeben der niedergelassenen Tätigkeit geführt haben und inwieweit zukünftige Hausärzte von diesen Problemen bedroht sind und was sie dagegen tun können, dürften für viele junge Ärzte die Entscheidung zu einer Niederlassung als Hausarzt erleichtern.

6 Praxen, die vollständig (Räumlichkeiten und Personal) von den Kassenärztlichen Vereinigungen betrieben werden. Der Hausarzt wird angestellt. 


\section{Zusammenfassung}

Einleitung: Hauptursache einer drohenden hausärztlichen Unterversorgung in ländlichen Gebieten ist ein Mangel an jungen Ärzten, die sich sowohl für eine hausärztliche Tätigkeit als auch deren Ausübung in ländlichen Gebieten entscheiden. Um dieser Entwicklung entgegenzuwirken, könnten biographische Erfahrungen neu niedergelassener Hausärzte hilfreich sein.

Studienfrage: Welche Erfahrungen, insbesondere zu Ausbildungszeiten, veranlassen junge Hausärzte zu einer Niederlassung im ländlichen Raum? Welche Faktoren sind förderlich oder hemmend in diesem Entscheidungsprozess?

Methoden: Im Rahmen eines qualitativen Studiendesigns führten wir 24 narrative Einzelinterviews mit Hausärzten, die sich in den letzten fünf Jahren in ländlichen Gebieten Niedersachsens niedergelassen hatten. Die Auswahl der Interviewteilnehmer erfolgte nach den Prinzipien „Maximum Variation" und dem „theoretischen Sampling". Die jeweils nächsten Interviewpartner ergaben sich anhand der parallel ausgewerteten Interviews und der daraus generierten vorläufigen Hypothesen. Diese sollten dann durch ähnliche oder kontrastierende Fälle untersucht werden. Die Datenanalyse der transkribierten Interviews erfolgte mit der Methode der Grounded Theory.

Ergebnisse: In fast allen Interviews artikulierte sich ein starkes Bedürfnis nach Lebensqualität. Bei den Motiven zur Facharztausbildung zeigte sich ein Spannungsfeld: Negative Erfahrungen in den Ausbildungsabschnitten und besonders in der Tätigkeit im Krankenhaus wirkten abschreckend. Positive Faktoren, besonders die Erhöhung der individuellen Lebensqualität durch Abbau beruflicher Stressoren, Zugewinn an persönlichen Ressourcen und Vereinbarkeit von Familie und Beruf ließen die Tätigkeit als Hausarzt attraktiver erscheinen. Gebremst wurden die zukünftigen Hausärzte bereits vor Antritt des Studiums durch die Abiturnote.

Schlussfolgerung: Ein frühzeitiger Kontakt mit dem Berufsbild „Hausarzt“ und Alternativen zur Abiturnote als Zulassungsberechtigung könnten einen Schritt zur Förderung einer hausärztlichen Berufstätigkeit darstellen. Beachtung verdient die Verbesserung der Familienkompatibilität und Lebensqualität mit Hilfe von sicherheitsfördernden Faktoren wie z. B. eine deutliche Minimierung des finanziellen Risikos. 


\section{Literaturverzeichnis ${ }^{7}$}

1. Adler G, Knesebeck J (2011): Ärztemangel und Ärztebedarf in Deutschland? Bundesgesundheitsblatt Gesundheitsforschung Gesundheitsschutz 54, 228-237

2. Angerer $P$, Petru R, Weig $M$, Glaser J: Arbeitsbedingungen und Befinden von Ärztinnen und Ärzten; In: Arbeitsbedingungen und Befinden von Ärztinnen und Ärzten: Befunde und Interventionen (Report Versorgungsforschung 2); hrsg. v. Angerer P, Schwartz FW.; Deutscher Ärzte-Verlag, Köln 2010, 175-184

3. Angerer P, Schwartz FW (Hrsg.) (2010): Arbeitsbedingungen und Befinden von Ärztinnen und Ärzten: Befunde und Interventionen (Report Versorgungsforschung 2); Deutscher Ärzte-Verlag, Köln 2010

4. Annan N, Maagaard R, Joos S, Ledig T, Steinhäuser J (2010): Wie könnte ein Train the Trainer Programm aussehen?: Eine Internet-Recherche. Z Allgemeinmed 86, 444-449

5. Approbationsordnung für Ärzte (ÄApprO 2002) (vom 27. Juni 2002 (BGBI. I S. 2405), die zuletzt durch Artikel 2 der Verordnung vom 2. August 2013 (BGBI. I S. 3005) geändert worden ist.

http://www.gesetze-im-internet.de/_appro_2002/BJNR240500002.html (23.06.2014)

6. Arbeitsgruppe Bielefelder Soziologen (Hrsg.) (1976): Kommunikative Sozialforschung: Alltagswissen und Alltagshandeln, Gemeindemachtforschung, Polizei, politische Erwachsenenbildung; Fink, München 1976

7. Ärztekammer Niedersachsen: Weiterbildungsordnung der Ärztekammer Niedersachsen: WBO 2013

8. Bärnighausen T, Bloom DE (2009): Financial incentives for return of service in underserved areas: a systematic review. BMC Health Serv Res $\underline{9}, 86$

9. Beske F, Golbach U, Drabinski T: Leistungskatalog des Gesundheitswesens im internationalen Vergleich (Schriftenreihe); Schmidt \& Klaunig, Kiel 2005

\footnotetext{
${ }^{7}$ Bildschirmausdrucke der im Inhaltverzeichnis angegebenen Internetquellen sind auf den Seiten 98 bis 105 zu finden.
} 
10. Böhm A: Theoretische Codieren: Textanalyse in der Grounded Theory; In: Qualitative Forschung: Ein Handbuch; hrsg. v. Kardorff Ev, Steinke I, Flick U, 9. Auflage; Rowohlt, Reinbek 2012, 475-485

11. Bojanowski S, Lange S, Laube A, Schübel J, Seele K, Bergmann A (2013): 2. Summerschool Allgemeinmedizin. Z Sozialisationsforsch Erziehungssoziol 89, 117-119

12. Bovier PA, Perneger TV (2003): Predictors of work satisfaction among physicians. Eur J Public Health $\underline{13}, 299-305$

13. Bundesärztekammer (2002): I,8: Dem deutschen Gesundheitswesen gehen die Ärzte aus: Beschlussprotokoll 105. Dt. Ärztetag 2002: Zu Punkt I der Tagesordnung: Gesundheits-, Sozial- und ärztliche Berufspolitik. http://www.bundesaerztekammer.de /page.asp?his=0.2.23.2450.2451.2459 (04.02.2012)

14. Bundesärztekammer (2013): Ergebnisse der Ärztestatistik zum 31. Dezember 2012: Kein Widerspruch - Ärztemangel trotz moderat steigender Arztzahlen. http://www.bundesaerztekammer.de/page.asp?his=0.3.11372 (04.02.2012)

15. Bundesgesetzblatt: Gesetz zur Verbesserung der Versorgungsstrukturen in der gesetzlichen Krankenversicherung (GKV-Versorgungsstrukturgesetz - GKV-VStG. Jahrgang 2011, Teil I, Nr. 70, Bonn 2011

16. Bundesministerium für Gesundheit: Eine flächendeckende bedarfsgerechte medizinische Versorgung bleibt auch in Zukunft in ganz Deutschland gesichert: Nr.58 2011 a

17. Bundesministerium für Gesundheit: Drucksache 862/11; Erste Verordnung zur Änderung der Approbationsordnung für Ärzte 2011 b

18. Bundesministerium für Gesundheit: Drucksache 238/12 zur Grunddrucksache 862/11; erste Verordnung zur Änderung der Approbationsordnung für Ärzte 2012 a

19. Bundesministerium für Gesundheit: Erste Verordnung zur Veränderung der Approbationsordnung: Wann ändert sich was? $2012 \mathrm{~b}$ http://www.google.de/url?sa=t\&rct=j\&q=\&esrc=s\&source=web\&cd=1\&ved=0CC8QFj AA\&url=http\%3A\%2F\%2Flsjv.rlp.de\%2Ffileadmin\%2Flsjv\%2Fdownloads\%2FGesundhe it\%2FLandespruefungsamt\%2FHumanmedizin\%2FAEnderung_der_Approbationsordn ung_fuer_AErzte_Wann_aendert_sich_was.pdf\&ei=EgbMUpiBI8LCtQb-r4GIBw\&usg= 
AFQjCNE7Kw3iUsng5n4-Ff91bTWjMGI_Wg\&bvm=bv.58187178,d.Yms\&cad=rja.

(04.02.2012)

20. Burgess RG: Sponsors, gatekeepers, members, and friends; In: Experiencing fieldwork: An inside view of qualitative research; hrsg. v. Shaffir W, Stebbins RA; Sage, Newbury Park 1991, 43-52

21. Cook G (1990): Trancribing infinity: Problems of context presentation. J Pragmatics 14, $1-24$

22. Daniels ZM, Van Leit BJ, Skipper BJ, Sanders ML, Rhyne RL (2007): Factors in Recruiting and Retaining Health Professionals for Rural Practice. J Rural Health $\underline{23}, 62-71$

23. Denzin, N. K., Lincoln, Y. S. (Hrsg.) (1994): Handbook of qualitative research; Sage, Thousand Oaks 1994

24. DEGAM [Deutsche Gesellschaft für Allgemeinmedizin]: Vorankündigung 3. Summerschool Allgemeinmedizin: für Medizinstudierende. vom 28. August bis 03. September 2013 in Greifswald 2013 http://www.degam.de/summerschool-2013.html, (04.02.2014)

25. DEGAM [Deutsche Gesellschaft für Allgemeinmedizin] (2014): Summerschool Allgemeinmedizin 2014 in Münster. http://www.degam.de/nachrichtendegam/items/summerschool-allgemeinmedizin-2014-in-muenster.html (04.02.2012)

26. Donner-Banzhoff N, Lichte T, Joos S, Steinhäuser J, Peters-Klimm F, Roos M, Löckermann $\mathrm{H}$, Haffner $\mathrm{C}$, Mühlenfeld $\mathrm{H}$, Nieswand $\mathrm{M}$ et al.: Verbundweiterbildung zum Allgemeinarzt: Ein Leitfaden für die Planung, Einrichtung und Führung von Weiterbildungsverbünden (DEGAM Schriften), 2. Auflage 2009, Frankfurt 2009

27. Fischer-Rosenthal W, Rosenthal G (1997): Warum Biographieanalyse und wie man sie macht. Z Sozialisationsforsch Erziehungssoziol 17, 405-427

28. Der neue Fischer Weltalmanach 2013: Zahlen Daten Fakten (72013); Fischer, Frankfurt am Main 2012

29. Fuchs-Heinritz W: Biographische Forschung: Eine Einführung in Praxis und Methoden; Westdeutscher Verlag, Opladen 1984 
30. Glaser BG: Theoretical sensitivity (Advances in the methodology of grounded theory); Sociology Press, Mill Valley 1978

31. Glaser BG, Strauss AL: The discovery of grounded theory: Strategies for qualitative research; Aldine Publishing, Chicago 1967

32. Haffke D: KVN gründet erste eigene Praxis im Emsland (Informationen der Kassenärztlichen Vereinigung Niedersachsen) Hannover 2013

33. Halaas GW, Zink T, Finstad D, Bolin K, Center B (2008): Recruitment and Retention of Rural Physicians: Outcomes From the Rural Physician Associate Program of Minnesota. J Rural Health $\underline{24}, 345-352$

34. Hausärzteverband Baden-Württemberg: Bewerbung zur 1. Schwarzwälder Winterschool in Hinterzarten, Stuttgart 2013

35. Henry JA, Edwards BJ, Crotty B (2009): Why do medical graduates choose rural careers? Rural Remote Health $\underline{9}, 1083$

36. Hibbeler B (2013): Initiative „Pro Quote Medizin“: Ärztinnen fordern feste Frauenquote. Dtsch Arztebl 110, 4

37. Hildenbrand B: Anselm Strauss; In: Qualitative Forschung: Ein Handbuch; hrsg. v. Kardorff Ev, Steinke I, Flick U, 9. Auflage; Rowohlt, Reinbek 2012, 32-42

38. Hopf C: Qualitative Interviews- ein Überblick; In: Qualitative Forschung: Ein Handbuch; hrsg. v. Kardorff Ev, Steinke I, Flick U, 9. Auflage; Rowohlt, Reinbek 2012, 349360

39. Jacob R, Heinz A, Müller C: Berufsmonitoring Medizinstudenten 2010: Ergebnisse einer bundesweiten Befragung; Deutscher Ärzte-Verlag, Köln 2012

40. Kardorff, E. V., Steinke, I., Flick, U. (Hrsg.) (2012): Qualitative Forschung: Ein Handbuch, 9. Auflage; Rowohlt Verlag, Reinbek 2012

41. Kassenärztliche Bundesvereinigung: Pressemitteilungen 2010: Im Jahr 2020 gibt es 7.000 Hausärzte weniger, Berlin 2010

42. Kassenärztliche Vereinigung Niedersachsens (2013): Niederlassen in Niedersachsen. http://www.kvn.de/icc/internet/nav/e1e/e1e70363-b94e-4821-b7d8- 
f51106fa453d\&sel_uCon=2532c76a-1a51-241d-e0d4-

515b8ff6bcbb\&uTem=aaaaaaaa-aaaa-aaaa-aaaa-000000000042.htm (03.02.2014)

43. Kassenärztliche Vereinigung Sachsen: Programm zur Durchführung der Studienbeihilfe; Kassenärztliche Vereinigung Sachsen, 0.0. 2009

44. Kassenärztliche Vereinigung Sachsen: Auch künftig Studienbeihilfe für Medizinstudenten, Dresden 2012

45. Kirchhoff T: Sehnsucht nach Natur: Über den Drang nach draußen in der heutigen Freizeitkultur (Edition Kulturwissenschaft Bd. 15); Transcript, Bielefeld 2012

46. Klose J, Rehbein I: Ärzteatlas 2011: Daten zur Versorgungsdichte von Vertragsärzten, 1. Auflage; Wissenschaftliches Institut der AOK, Berlin 2011

47. Klose J, Uhlemann T, Gutschmidt S: Ärztemangel - Ärzteschwemme?: Auswirkungen der Altersstruktur von Ärzten auf die vertragsärztliche Versorgung; Wissenschaftliches Institut der AOK, Bonn 2003

48. Klose J, Rehbein I, Uhlemann T: Ärzteatlas: Daten zur Versorgungsdichte von Vertragsärzten, 1. Auflage; WIdO, Wissenschaftliches Institut der AOK, Bonn 2007

49. Köhler K (2009): "Studienbeihilfe" für Medizinstudenten: Erste Medizinstudenten unterschreiben für Sachsen. Arztebl Sachsen 2009,VI, 259

50. Kopetsch T: Dem deutschen Gesundheitswesen gehen die Ärzte aus!: Studie zur Altersstruktur- und Arztzahlentwicklung, 5. Auflage; Bundesärztekammer und Kassenärztliche Bundesvereinigung, Berlin 2010

51. Kowal S, O'Connell DC: Zur Transkription von Gesprächen; In: Qualitative Forschung: Ein Handbuch; hrsg. v. Kardorff Ev, Steinke I, Flick U, 9. Auflage; Rowohlt, Reinbek 2012, 437-447

52. Louv R: The nature principle: Human restoration and the end of nature-deficit disorder, 1. Auflage; Algonquin Books of Chapel Hill, Chapel Hill 2011

53. Lu DJ, Hakes J, Bai M, Tolhurst H, Dickinson JA (2008): Rural intentions: factors affecting the career choices of family medicine graduates. Can Fam Physician $\underline{54}$, 10161017.e5 
54. Merkens H: Auswahlverfahren, Sampling, Fallkonstruktion; In: Qualitative Forschung: Ein Handbuch; hrsg. v. Kardorff Ev, Steinke I, Flick U, 9. Auflage; Rowohlt, Reinbek 2012, 286-299

55. Mihm A (2010): Rösler plant "Landarztquote" für Medizinstudenten. FAZ, 05 April 2010

56. Morse JM: Designing funded qualitative research; In: Handbook of qualitative research; hrsg. v. Denzin NK, Lincoln YS; Sage Thousand Oaks 1994, 220-235

57. Niehus H, Berger B, Stamer M, Schmacke N: Die Sicherung der hausärztlichen Versorgung in der Perspektive des ärztlichen Nachwuchses und niedergelassener Hausärztinnen und Hausärzte, Bremen 2008

58. N24 - Gesellschaft für Nachrichten und Zeitgeschehen (2014): Mehr Landärzte und kürzere Wartezeiten. http://www.n24.de/n24/Nachrichten/Politik/d/4077282/mehrlandaerzte-und-kuerzere-wartezeiten.html (03.02.2014)

59. Oberlander W: Die berufliche Situation junger Ärztinnen und Ärzte; In: Arbeitsbedingungen und Befinden von Ärztinnen und Ärzten: Befunde und Interventionen (Report Versorgungsforschung 2); hrsg. v. Angerer P, Schwartz FW.; Deutscher Ärzte-Verlag, Köln 2010, 117-125

60. Rabinowitz HK, Paynter NP (2002): MSJAMA. The rural vs urban practice decision. JAMA $\underline{287}, 113$

61. Reeh T: Natur erleben und Raum inszenieren (Universitätsdrucke Göttingen Bd. 4); Universitäts-Verlag Göttingen, Göttingen 2008

62. Richter-Kuhlmann E (2007): „Ja“ zum Arztberuf, „Nein“ zu deutschen Verhältnissen: Die heutigen Studierenden wollen kurativ tätig sein - nicht unbedingt jedoch in Deutschland. Nur zwei von drei Studenten sehen gute Berufschancen an deutschen Krankenhäusern: Ergebnisse einer Umfrage des Deutschen Ärztesblattes Studieren.de. Dtsch Arztebl $\underline{104}, 1881-1882$

63. Rosenthal G: Interpretative Sozialforschung: Eine Einführung (Grundlagentexte Soziologie); Juventa, Weinheim 2005 
64. Schäfer H, Gündling P, Gilbert K, Sennekamp M, Mesenholl-Strehler E, Gerlach F (2008): Das praktische Jahr im Fach Allgemeinmedizin - Erste Ergebnisse und Erfahrungen an der Universität Frankfurt/Main. Z Allgemeinmed 84, 201-206

65. Schäfer H, Sennekamp M, Güthlin C, Krentz H, Gerlach FM (2009): Kann das Blockpraktikum Allgemeinmedizin zum Beruf des Hausarztes motivieren? Z Allgemeinmed 85, 206-209

66. Schmidt C, Möller J, Schmidt K, Gerbershagen M, Wappler F, Limmroth V, Padosch S, Bauer M (2011): Generation Y. Anaesthesist 60 , 517-524

67. Schütze F: Zur Hervorlockung und Analyse von Erzählungen thematisch relevanter Geschichten im Rahmen soziologischer Feldforschung: dargestellt an einem Projekt zur Erforschung von kommunalen Machtstrukturen; In: Kommunikative Sozialforschung: Alltagswissen und Alltagshandeln, Gemeindemachtforschung, Polizei, politische Erwachsenenbildung; hrsg. v. Arbeitsgruppe Bielefelder Soziologen; Fink, München 1976, 159-259

68. Shaffir, W., Stebbins, R. A. (Hrsg.) (1991): Experiencing fieldwork: An inside view of qualitative research; Sage, Newbury Park 1991

69. Fünftes Buch Sozialgesetzbuch: Gesetzliche Krankenversicherung (Artikel 1 des Gesetzes vom 20. Dezember 1988, BGBI. I S. 2477), das zuletzt durch Artikel 1 des Gesetzes vom 22. Dezember 2013 (BGBI. I S. 4382) geändert worden ist. http://www.sozialgesetzbuch-sgb.de/sgbv/1.html (23.06.2014)

70. Steger T, Langosch C, Klement A, Onnasch J (2012): „Klasse Allgemeinmedizin“: ein Lehrkonzept für zukünftige Landärzte. Z Allgemeinmed $\underline{88}$, 264-267

71. Steinhäuser J, Ledig T, Szecsenyi J, Eicher C, Engeser P, Roos $M$, Bungartz J, Joos $S$ (2012): Train the Trainer für weiterbildungsbefugte Allgemeinärzte: Ein Bericht über die Pilotveranstaltung im Rahmen des Programms Verbundweiterbildungplus. GMS Z Med Ausbild 29, 1-12

72. Steinhäuser J, Joos S, Szecsenyi J, Götz K (2013): Welche Faktoren fördern die Vorstellung sich im ländlichen Raum niederzulassen? Z Allgemeinmed $\underline{89}, 10-15$

73. Strauss AL: Qualitative analysis for social scientists; Cambridge University Press, Cambridge 1987 
74. Strauss AL: Grundlagen qualitativer Sozialforschung: Datenanalyse und Theoriebildung in der empirischen soziologischen Forschung (UTB für Wissenschaft 1776), 2. Auflage; Fink, München 1994

75. Strauss AL, Corbin JM: Grounded theory: Grundlagen qualitativer Sozialforschung; Beltz, Weinheim 1996

76. University of Minnesota (2012): About RPAP - MED - Rural Physician Associate Program, University of Minnesota. http://www.med.umn.edu/RPAP/about/home.html (03.02.2014)

77. University of Minnesota Academic Health Center (2010): University of Minnesota Medical School: Duluth Campus.

http://www.youtube.com/watch?v=K4pW95kiOTw\&feature=youtube_gdata_player (03.02.2014)

78. van den Berg N, Meinke C, Heymann R, Fiß T, Suckert E, Pöller C, Dreier A, Rogalski H, Karopka T, Oppermann R et al. (2009): AGnES: Hausarztunterstützung durch qualifizierte Praxismitarbeiter - Evaluation der Modellprojekte: Qualität und Akzeptanz. Dtsch Arztebl 2009, 3-9

79. Vereinbarung zur Förderung der Weiterbildung in der Allgemeinmedizin in der ambulanten und stationären Versorgung. Vertragspartner: Deutsche Krankenhausgesellschaft (DKG), Kassenärztliche Bundesvereinigung (KBV), Spitzenverband Bund der Krankenkassen (GKV-Spitzenverband). Grundlage: Artikel 8 Abs. 2 GKV-SolG i. d. F. des GKV-OrgWG v. 15.12.2008. In Kraft getreten am 01.01.2010

80. Wollny A, Marx G (2009): Qualitative Sozialforschung- Ausgangspunkte und Ansätze für eine forschende Allgemeinmedizin: Teil 2: Qualitative Inhaltsanalyse vs. Grounded Theory. Z Allgemeinmed $\underline{85}, 467-476$

81. Zink T, Center B, Finstad D, Boulger JG, Repesh LA, Westra R, Christensen R, Brooks KD (2010): Efforts to Graduate More Primary Care Physicians and Physicians Who Will Practice in Rural Areas: Examining Outcomes From the University of MinnesotaDuluth and the Rural Physician Associate Program. Acad Med 85, 599-604 


\section{Abbildungsverzeichnis ${ }^{8}$}

Abbildung 1 Gesamtversorgungsgrad mit Hausärzten nach KVen 2010 ........................................... 4

Abbildung 2 Qualitative Datenerhebung und- analyse als zirkulärer Prozess .................................... 18

Abbildung 3 Grounded Theory als triadischer und zirkulärer Prozess ................................................. 23

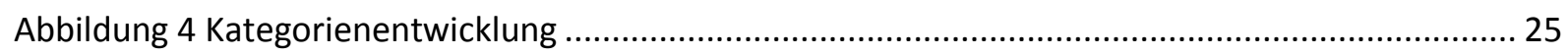

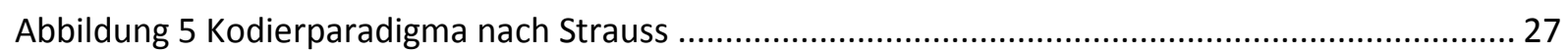

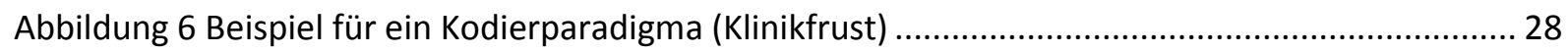

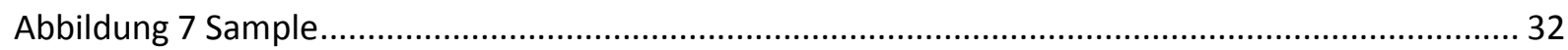

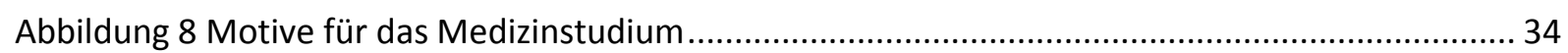

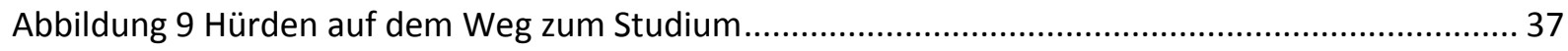

Abbildung 10 Motive und Gegengründe für die Facharztwahl ........................................................ 40

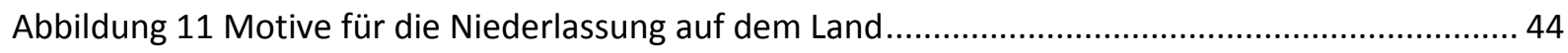

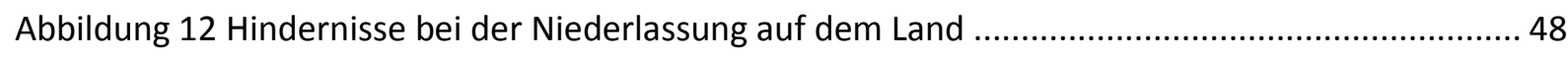

\section{Tabellenverzeichnis}

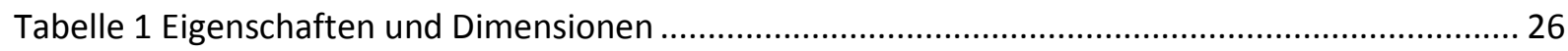

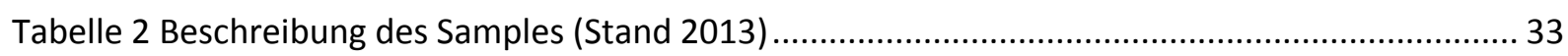

${ }^{8}$ Die Verwendung von Abbildung 1 erfolgt mit freundlicher Genehmigung von J. Klose. Der Abdruck der Abbildungen 3 und 5 erfolgt mit freundlicher Genehmigung des Rowohlt Taschenbuch Verlages. 


\section{Anhang}

\subsection{Anlage 1: 1. Anschreiben}

\section{UNIVERSITÄTSMEDIZIN $=$
GÖTTINGEN $=0$}

Universitätsmedizin Göttingen, 37099 Göttingen

Komm. Leitung Abteilung Allgemeinmedizin

\author{
Abteilung Allgemeinmedizin \\ Prof. Dr. W. Himmel \\ 37099 Göttingen Briefpost \\ Humboldtallee 38, 37073 Göttingen Adresse \\ 0551 / 39-22648 Telefon \\ 0551 / 39-9530 Fax \\ whimmel@gwdg.de E-Mail
}

\section{Hausärztlicher Nachwuchs in Niedersachsen}

\section{Sehr geehrter Herr Kollege}

die Abt. Allgemeinmedizin der Universitätsmedizin Göttingen engagiert sich in der Aus- und Weiterbildung zukünftiger Hausärzte. Dabei liegt uns die Situation der hausärztlichen Versorgung in unserem Bundesland besonders am Herzen. Unser Ziel ist es, Konzepte zu entwickeln, die dem prognostizierten Hausärztemangel in Niedersachsen entgegenwirken. Dies tun wir gemeinsam mit der KV Niedersachsen und bitten um Ihre Unterstützung.

Wir möchten von niedergelassenen Kolleginnen und Kollegen erfahren, welche Gründe sie zu einer Niederlassung vor allem in ländlicheren bzw. kleinstädtischen Gebieten veranlasst und welche Erfahrungen sie bisher gemacht haben. Wir konzentrieren uns dabei auf die Hausärzte, die sich in den letzten 5 Jahren in unserem Bundesland niedergelassen haben. Ihre Sichtweise, Meinung und Einschätzung ist uns besonders wichtig.

Mit einigen Kolleginnen und Kollegen möchten wir daher gern ein persönliches Gespräch führen und sie in ihrer Praxis besuchen. Erfahrungsgemäß dauert dieses Gespräch zwischen 30 und 45 min. Auch eine kleine Aufwandsentschädigung in Höhe von $80 €$ können wir Ihnen dafür anbieten. Wir bitten Sie, das beigefügte Formular per Fax an uns zurückzusenden. Selbstverständlich stehen wir Ihnen für Fragen und Anregungen jederzeit gern zur Verfügung. Ihre Ansprechpartnerin ist Celia Richter (celia.richter@med.uni-goettingen.de, Tel.0551-39-22638).

Mit freundlichen kollegialen Grüßen 


\section{Faxantwort}

an Dr. med. Celia Richter

Abteilung Allgemeinmedizin

Universitätsmedizin Göttingen

0551/39-9530

Forschungsprojekt „Niederlassung als Hausärztin/Hausarzt in Niedersachsen“

$\square \quad$ Ja, ich möchte an dem persönlichen Gespräch teilnehmen.

$\square \quad$ Nein, ich habe kein Interesse (evtl. mögen Sie uns einen Grund nennen).

$\square \quad$ Im Moment habe ich kein Interesse. Bitte sprechen Sie mich zu einem späteren Zeitpunkt noch einmal an.

$\square \quad$ Ich bitte um mehr Informationen. Rufen Sie mich doch zurück, am besten:

Name:

Ort:

Telefon:

Fax: 


\subsection{Anlage 2: biographischer Fragebogen}

\section{$\begin{aligned} \text { UNIVERSITÄTSMEDIZIN } & =1 \\ \text { GÖTTINGEN } & =0\end{aligned}$}

Universitätsmedizin Göttingen, 37099 Göttingen

Direktorin der Abteilung Allgemeinmedizin

Prof. Dr. med. Eva Hummers-Pradier, Humboldtallee 38
Abteilung Allgemeinmedizin Prof. Dr. W. Himmel

37099 Göttingen Briefpost Humboldtallee 38, 37073 Göttingen Adresse 0551 / 39-22648 Telefon 0551 / 39-9530 Fax whimmel@gwdg.de E-Mail

\section{Forschungsprojekt „Niederlassung als Hausärztin/Hausarzt in Niedersachsen“}

Sehr geehrte Herr Kollege,

wir danken Ihnen für Ihre positive Rückmeldung. Bereits jetzt hat uns mehr als die Hälfte der angeschriebenen Hausärztinnen und Hausärzte ihr Interesse an der Studie mitgeteilt. Wir freuen uns sehr darüber!

In einem ersten Schritt möchten wir mit 15 bis 20 Hausärztinnen und -ärzten ein persönliches Gespräch führen, bei dem sicher auch Aspekte zur Sprache kommen, die in der bisherigen Diskussion und Forschung zum Hausärztemangel kaum oder gar nicht berücksichtigt wurden. Um für diese erste Runde der Interviews die Charakteristika der Interviewpartnerinnen und -partner möglichst breit zu fächern, bitten wir Sie, den beigefügten Fragebogen zu beantworten. Auf diese Weise können wir die Interviewpartnerinnen und -partner entsprechend ihres persönlichen Hintergrundes möglichst ausgewogen und kontrastreich auswählen.

Bitten senden Sie den Fragebogen in dem beiliegenden frankierten Rückumschlag oder als Fax an Dr. Celia Richter. Ihre Daten werden nur im Rahmen dieses Forschungsprojektes verwendet, die personenbezogenen Daten werden nicht an Dritte weitergegeben und nach Durchführung der Interviews gelöscht.

Für Fragen stehen wir Ihnen jederzeit gerne persönlich zur Verfügung!

Mit herzlichen Grüßen aus Göttingen 
Anlage

\section{Fragebogen zum Projekt \\ „Perspektive: Niederlassung als Hausärztin/Hausarzt \\ in Niedersachsen"}

Sollten Sie auf eine der folgenden Fragen lieber nicht antworten wollen, so lassen Sie die betreffende Frage bitte einfach aus! Bitte senden Sie den ausgefüllten Fragebogen in dem beiliegenden frankierten Rückumschlag zurück. Alternativ können Sie ihn auch gerne per Fax senden an 0551-399530. Herzlichen Dank!

Name: Vorname:

Geburtsjahr:

Geschlecht: $\square_{\text {männlich } \quad \square \text { weiblich }}$

Wo sind sie aufgewachsen?

\section{Wo haben sie studiert?}

In welchen Orten/Regionen waren

Sie vor Ihrer derzeitigen Niederlassung

tätig?

Ort der derzeitigen Niederlassung:

War ihnen bereits während des

Studiums klar, dass Sie Hausärztin/-arzt $\quad \square$ ja $\quad \square$ nein werden wollen?

Was ist ihr Familienstand?

$\begin{array}{ll}\square \text { ledig } & \square \text { verheiratet } \\ \square_{\text {verwitwet }} & \square \text { in fester Partnerschaft lebend } \\ \text { geschieden } & \end{array}$

Falls sie in einer festen Partnerschaft leben, welchen Beruf geht Ihre

Partnerin/Ihr Partner nach?

Haben sie Kinder? $\quad \square_{\text {ja }} \quad \square_{\text {nein }}$

Wie alt sind Ihre Kinder? Kind1:___ Kind2:__ Kind3:

Welchen Beruf üben bzw. übten ihre Eltern aus?

Mutter:

Vater: 


\title{
11.3 Anlage 3: Informationsblatt
}

\section{Forschungsprojekt}

\author{
„Perspektive: Niederlassung als Hausärztin/Hausarzt \\ in Niedersachsen" \\ - Informationsblatt -
}

Das Projekt wird vom Bundesministerium für Bildung und Forschung finanziert. Die erhobenen Daten werden von den Projektmitarbeiterinnen und -arbeitern der Abt. für Allgemeinmedizin der Universitätsmedizin Göttingen sorgsam behandelt und ausgewertet. Laut den Datenschutzbestimmungen benötigen wir zur Speicherung und Auswertung des mit Ihnen geführten Interviews Ihre ausdrückliche Einwilligung.

Die verantwortliche Leitung des Projektes liegt bei

- Prof. Dr. Wolfgang Himmel (Akademischer Oberrat der Abt. Allgemeinmedizin, Universitätsmedizin Göttingen) und

- Dr. med. Celia Richter (wissenschaftliche Mitarbeiterin der Abt. Allgemeinmedizin, Universitätsmedizin Göttingen).

Hauptziel dieses Forschungsprojekts ist es, die Motive und Erfahrungen von Hausärztinnen und ärzten für und bei der Niederlassung in Niedersachsen zu erfahren. Dazu möchten wir niedergelassene Allgemeinärztinnen und -ärzte sowie hausärztlich tätige Internistinnen und Internisten befragen. Aus den im Projekt erzielten Erkenntnissen werden wir Handlungsempfehlungen erarbeiten und den gesundheitspolitischen Entscheidungsträgern zur Verfügung stellen. 
Das Forschungsprojekt dient allein wissenschaftlichen Zwecken. Die Durchführung der Studie geschieht unter Berücksichtigung der Bestimmungen des Bundesdatenschutzgesetzes. Die Interviewerin unterliegt der Schweigepflicht und ist zur Einhaltung des Datengeheimnisses verpflichtet.

Um Ihre Anonymität zu sichern und die Datenschutzvorschriften zu erfüllen, sichern wir Ihnen folgende Verfahrensweise zu:

- Wir gehen sorgfältig mit Ihrem Erzählten um: Wir zeichnen das Gespräch auf einem elek-tronischen Datenträger auf. Die Aufnahme wird pseudonymisiert transkribiert, anschließend wird der Datenträger gelöscht.

- Pseudonymisierung heißt: wir verändern alle Personen-, Ort-, Straßennamen und alle Altersangaben.

- Ihr Name und Ihre Telefonnummer werden am Ende des Projektes in unseren Unterlagen gelöscht, sodass lediglich eine pseudonymisierte Abschrift des Interviews existiert. Die von Ihnen unterschriebene Erklärung zur Einwilligung in die Auswertung wird in einem gesonderten Ordner an einer gesicherten Stelle aufbewahrt, welche nur für die Projektleitung zugänglich ist. Die Aufbewahrung Ihrer Einwilligungserklärung dient lediglich dazu, bei einer Überprüfung durch den Datenschutzbeauftragten nachweisen zu können, dass Sie mit der Auswertung einverstanden sind. Sie kann mit Ihrem Interview nicht mehr in Verbindung gebracht werden.

- Die Abschrift wird nicht veröffentlicht und ist nur für die projektinterne Auswertung bestimmt. Die Projektmitarbeiterinnen und -arbeiter, welche die pseudonymisierte Abschrift lesen, unterliegen ebenfalls der Schweigepflicht. In späteren Veröffentlichungen zur Studie werden unter Umständen einzelne Zitate Ihres Interviews verwendet, welche selbstverständlich keine Rückschlüsse auf Ihre Person zulassen.

- Die Abschrift wird in der Abt. für Allgemeinmedizin der Universitätsmedizin Göttingen archiviert und unter Einhaltung der bisher genannten Vorschriften ggf. anderen Projekten als Sekundärmaterial zur Verfügung gestellt.

Gemäß den Datenschutzbestimmungen möchten wir Ihnen versichern, dass Ihnen aus einer Nichtteilnahme an der Studie keine Nachteile entstehen. Sie können auch bei einzelnen Fragen Ihre Antwort verweigern. Ihre Einwilligung zur Teilnahme am Forschungsprojekt ist freiwillig und kann jederzeit von Ihnen widerrufen werden. Auf Ihren Wunsch erfolgt die Löschung des Interviews in einem solchen Fall umgehend.

Wir bedanken uns für Ihre Teilnahme und Ihr Engagement, und hoffen, dass die Ergebnisse unseres Projektes dazu beitragen, die hausärztliche Versorgung von Patientinnen und Patienten in Niedersachsen nachhaltig zu verbessern. 


\subsection{Anlage 4: Einwilligungserklärung}

\section{UNIVERSITÄTSMEDIZIN \\ GÖTTINGEN

ID:

\section{Einwilligungserklärung}

Ich erkläre mich bereit, im Rahmen des Forschungsprojekts „Perspektive: Niederlassung als Hausärztin/Hausarzt in Niedersachsen“ ein Interview zu geben.

Ich wurde über die Inhalte und Methoden des Projektes informiert. Ich nehme freiwillig an dem Interview teil. Ich wurde darüber informiert, dass alle erhobenen Daten zu meiner Person pseudonymisiert und zu rein wissenschaftlichen Zwecken genutzt werden. Die Tonbandaufnahmen werden nach Abschluss der Untersuchung gelöscht, lediglich diese Einwilligungserklärung wird für den Fall einer möglichen Überprüfung durch den Datenschutzbeauftragten aufbewahrt. Dieses Dokument wird jedoch nicht mit der pseudonymisierten Abschrift des Interviews zusammenführbar sein.

Ich stimme der Aufnahme und der Transkription des Interviews zu. Ich bin damit einverstanden, dass einzelne Sätze, die nicht mit meiner Person in Verbindung gebracht werden können, als Material für wissenschaftliche Zwecke und die Weiterentwicklung der Forschung genutzt werden können.

Ich bin mit den Rahmenbedingungen der wissenschaftlichen Untersuchung einverstanden. Ich wurde darüber informiert, dass ich meine Einwilligung jederzeit zurückziehen kann.

Eine Kopie dieser Erklärung habe ich erhalten. 


\subsection{Anlage 5: Erzählaufforderung}

\section{Projekt „Niederlassung und Berufszufriedenheit von Hausärzten: Interview} Interviewcode:

\section{Erzählaufforderung:}

„Ich interessiere mich für Ihre Lebensgeschichte. Bitte erzählen Sie mir Ihre Lebensgeschichte, all die Erlebnisse, die Ihnen dazu einfallen. Sie können sich so viel Zeit nehmen wie Sie möchten. Ich werde Sie auch erst einmal nicht unterbrechen, mir nur einige Notizen machen, auf die ich später zurückkomme."

\section{Notizen:}




\section{Entscheidende Abschnitte:}

Kindheit und Jugend, Studium, Entscheidung Hausarzt, Arztkarriere

\section{Externe Nachfragen:}

- „Bestimmt erinnern Sie sich noch an die Situation als Sie sich entschieden haben, sich hier niederzulassen. Können Sie mir bitte etwas über die Zeit erzählen?"

- „Können Sie sich an eine oder mehrere Situationen erinnern, in der Sie sich als Hausarzt richtig zufrieden gefühlt haben? Bitte erzählen Sie mir davon."

- „Können Sie sich an eine oder mehrere Situationen erinnern, in der Sie sich als Hausarzt richtig unzufrieden gefühlt haben? Bitte erzählen Sie mir davon."

- Möchten Sie mir noch etwas erzählen?

\section{Weitere Notizen:}




\subsection{Anlage 6: Interview-Memo}

GPS Niedersachsen - Memo zum Interview

Am:

04.05.2012

Beginn des Interviews:

11:00 Uhr

Ende des Interviews:

12:00 Uhr

Interview durch:

Annika Heubrock

Audiodatei:

$1: 23: 25$

Ort des Interviews:

Haus von 14_GPS_Gö_m_GP_0

Memo geschrieben am:

06.05.12, 13:00 Uhr, Stichpunkte bereits am 04.05.12

Ort:

Göttingen

Von:

Annika Heubrock

\section{Soziodemographische Daten}

Maskierter Name bzw. Pseudonym: 14_GPS_Gö_m_GP_0

Alter:

39

Geschlecht:

männlich

Kindheit:

09_M_Stadt_AK

Studium:

07_M_Stadt_B, 10_G_Stadt_D

vor der Niederlassung:

09_M_Stadt_AG,09_M_Stadt_AK

Niederlassung:

09_M_Stadt_AK

Familienstand:

verheiratet

Beruf des Partners:

Lehrerin

Kinder:

zwei $(4,6)$

Beruf der Mutter:

Lehrerin

Beruf des Vaters:

Lehrer

\section{Telefonat vom 27.04.12 um 9:30 Uhr}

Das erste Mal rief ich am 27.04.12 um 9:30 Uhr in der Praxis von 14_GPS_Gö_m_GP_0 an. Ich hatte eine Praxismitarbeiterin am Telefon, die mir direkt einen Termin geben wollte. Nachdem sie mir einen Termin für den kommenden Mittwoch um 11:00 Uhr zuwies, erkundigte ich mich noch einmal, ob ein einstündiges Gespräch wirklich in den Terminplan eines 
Mittwochvormittages passen würde. Dies verneinte sie und fragte mich dann, ob ich sie zwischen 12.00 Uhr und 12.15 Uhr am selbigen Tage zurückrufen könnte. Meinen Rückruf nahm erneut eine Praxismitarbeiterin entgegen, die über das Vorgespräch nicht informiert worden war, mich dann aber direkt mit 14_GPS_Gö_m_GP_ 0 verband. Ich schlug ihm einen Termin am kommenden Mittwochnachmittag vor, den er aber ablehnte. Er schlug daraufhin den kommenden Freitag vor. Bis 16:00 Uhr sei er an diesem Tag durchgehend erreichbar. Wir vereinbarten daraufhin den Termin um 11 Uhr in seiner Praxis. Falls es dort zu laut sein würde, könne man in sein Haus ausweichen.

\section{Gesprächssituation}

Als ich pünktlich zehn vor elf in der Praxis von 14_GPS_Gö_m_GP_ 0 eintraf, war 14_GPS_Gö_m_GP_0 selber nicht vor Ort. Eine der Arzthelferinnen - wie sich später herausstellte, die Frau des Praxis-Partners von 14_GPS_Gö_m_GP_0 - empfing mich eher unfreundlich und forderte mich auf, im Wartezimmer Platz zu nehmen. Die Praxisräumlichkeiten waren hell, aber unattraktiv. Die Wände waren zum Teil nicht mehr gut gestrichen und die Einrichtung war sicherlich schon einige Jahre alt. Beim Eintreten in die Praxis kam man direkt zu einem großen Empfangstresen, in dessen Hintergrund eine große Tafel an der Wand hing, auf der stand „Gefahr! Kein Tetanusschutz? Aber Hallo! Sprechen Sie uns an!“. Die Wortwahl dieses Textes fand ich etwas seltsam. Von der Empfangshalle gingen mehrere Zimmer ab, unter anderem auch das Wartezimmer. Dieses war ebenfalls hell, die Stühle und das Sofa sahen jedoch etwas abgegriffen aus. An der Decke befand sich eine große silberfarbene Raute mit eingelassenen Halogenstrahlern. Nach etwa 10 Minuten im Wartezimmer sprach mich eine andere Arzthelferin an und sagte mir, dass 14_GPS_Gö_m_GP_0 noch privat unterwegs sei. Er würde sich etwas verspäten und mich daher bitten, zu ihm nach Hause zu kommen. Die Arzthelferin erklärte mir kurz den Weg. 14_GPS_Gö_m_GP_0 wohnte direkt in einer Parallelstraße zur Praxis in einem Neubau. Wir trafen gleichzeitig an seinem Haus ein. 14_GPS_Gö_m_GP_0 war gut gelaunt und entschuldigte sich mehrfach, den Termin verschwitzt zu haben. Er bot mir direkt das „du“ an. Sein Haus war sehr modern, bei Eintritt kam man in einen großen offenen Wohn-Ess-Bereich, in dem Kinderspielzeug an diversen Stellen lag. Rundherum gab es fast ausschließlich Fensterfronten, weshalb das Haus sehr hell wirkte. 14_GPS_Gö_m_GP_0 stellte mir seine Frau (XX12) und seine Putzfrau kurz vor, bevor letztere sich verabschiedete. Seine Frau ging zurück in ihr Arbeitszimmer. Er bot mir Getränke an, 
bevor wir uns an einen großen Eichentisch setzten. Das Gespräch verlief absolut erfreulich. 14_GPS_Gö_m_GP_0 erzählte munter drauf los und schien offensichtlich Freude daran zu haben, jemanden an seinem Leben teilhaben zu lassen. Nach einer Weile kam XX12 zurück in die Küche und fragte, ob sie nebenbei kochen dürfte.14_GPS_Gö_m_GP_0 bejahte dies. Im weiteren Verlauf des Gesprächs warf somit XX12 noch die eine oder andere Bemerkung ein. Das ganze Gespräch empfand ich als äußerst angenehm und das Verhältnis schon fast als freundschaftlich. Gegen Ende des Gesprächs fand eine kurze Unterbrechung statt. 14_GPS_Gö_m_GP_0 musste XX12 zur Hand gehen, um Möbel aus dem PKW zu holen, damit die Kinder abgeholt werden konnten. Kurz vor Eintreffen der Kinder waren wir auch mit dem Interview fertig. 14_GPS_Gö_m_GP_0 und XX12 boten mir beide an, noch zum Essen zu bleiben, was ich aber dankend ablehnte. Die beiden Jungs der Familie waren sehr aufgeschlossen und erzählten mir direkt freudig, dass sie zum Mittag Caprisonne trinken dürften, wenn sie nicht den Papa stören würden. Wir waren zwar schon fertig, aber die Caprisonne gab's trotzdem.

\section{Zusammenfassung des Interviewinhaltes}

14_GPS_Gö_m_GP_0 wurde in 09_M_Stadt_AK geboren und ist auch dort behütet aufgewachsen. Sein Abitur hatte er ebenfalls in 09_M_Stadt_AK gemacht. Er wurde als Sohn eines Lehrerehepaares für Haupt- bzw. Realschule geboren. Er hat noch einen 5 Jahre jüngeren Bruder. Seine Eltern sind bereits seit ihrem 15. bzw. 18. Lebensjahr ein Paar. Sein Vater ist ein Aussiedler aus Bereich_BQ. Deshalb konnte dieser nicht im katholischen 09_M_Stadt_AK zur Schule gehen, sondern musste nach 09_K_Stadt_R, um hier eine evangelische Schule besuchen zu können. Anfänglich wollte sein Vater Tiermedizin studieren, was aber aufgrund von finanziellen Möglichkeiten und vorheriger Krankheit nicht möglich war. Daraufhin entschloss er sich, zusammen mit seiner Frau nach 09_M_Stadt_X zu gehen, um dort Lehramt zu studieren. Beide arbeiteten im Anschluss auch als Lehrer. Mittlerweile sind beide jedoch berentet und kümmern sich täglich um die Enkelkinder.

14_GPS_Gö_m_GP_0's Schulzeit verlief normal, er war ein „fauler Hund“ und hatte nie die Muße, besonders viel auswendig zu lernen. Da er zu Abiturzeiten noch den Wunsch hegte, einmal Sportjournalist zu werden, wählte er als Leistungskurse Sport und Deutsch. In der 11ten Klasse hatte er deshalb auch bereits ein Praktikum auf diesem Gebiet absolviert. Neben der Schule trieb er besonders viel Sport. Fußball, Tennis, aber auch Musik gehörten mit zu 
seinen Hobbys. Seine Kindheit in 09_M_Stadt_AK hatte 14_GPS_Gö_m_GP_0 in bester Erinnerung. Deshalb hatte er auch schon immer den Wunsch, seinen Kindern einmal so eine Jugend bieten zu können.

Nach dem Abitur machte 14_GPS_Gö_m_GP_0 seinen 1-jährigen Zivildienst in 09_M_Stadt_AK bei einer Hilfsorganisation. Hier absolvierte er auch seine Ausbildung zum Rettungssanitäter. Durch die Ausbildung und die damit verbundene Tätigkeit erwuchs in inm der Wunsch, Medizin zu studieren. Am Rettungsdienst mochte er besonders die abwechslungsreiche Tätigkeit - nie zu wissen, was auf einen zukommt und dann doch die Situation schnell in den Griff zu bekommen. Er merkte zwar auch den psychischen Druck, doch brachte ihm die Arbeit auch einen persönlichen Kick.

Nach dem Rettungsdienst hat 14_GPS_Gö_m_GP_0 dann ein Studium an der Sporthochschule in 10_G_Stadt_E begonnen. Er hatte zwar schon bei Antritt des Sportstudiums den Wunsch, Medizin zu studieren, war sich aber dennoch unsicher, da er eigentlich immer Sportjournalist werden wollte. Nach 3 Semestern Sportstudium, das für ihn körperlich, jedoch nicht geistig, eine Herausforderung war, stand dann die Entscheidung fest: dies ist der falsche Weg. Er beendete das Studium in 10_G_Stadt_E um in 07_M_Stadt_B Medizin zu studieren.

In 07_M_Stadt_B hatte 14_GPS_Gö_m_GP_0 eine tolle Zeit. Seit dem Präparier-Kurs, zu Anfang des Studiums, hatte er einen engen Freundeskreis, mit dem er allabendlich viel unternahm. Solche Freundschaften hatten ihm zuvor in 10_G_Stadt_E gefehlt. Er schätzte besonders die kleinstädtische Atmosphäre von 07_M_Stadt_B. Das Studium verlief für 14_GPS_Gö_m_GP_0 ohne Probleme. Er schaffte alle Scheine auf Anhieb. Lediglich die Doktorarbeit zog sich soweit hin, dass er sie am Ende parallel zum Arbeitsalltag hätte schreiben müssen, was er jedoch nicht mehr tat. Einen Doktorgrad erlangte er somit nie, was ihn im Nachhinein nur im Hinblick auf das verlorene Semester störte. Nach dem Staatsexamen ersehnte sich 14_GPS_Gö_m_GP_0 dann aber doch noch einen Ortwechsel. Er entschloss sich, das PJ in 10_G_Stadt_D zu machen. Zu dieser Zeit hatte 14_GPS_Gö_m_GP_0 noch eine Freundin, die in 09_G_Stadt_B studierte und an MS und Angststörungen litt. Diese besuchte er schon aus 07_M_Stadt_B regelmäßig und wollte deshalb durch den Umzug nach 10_G_Stadt_D die Distanz zu ihr verkürzen. Die Beziehung zerbrach jedoch nur kurz nach dem Umzug, da 14_GPS_Gö_m_GP_0 zu dem Zeitpunkt XX12 kennenlernte. Diese arbeitete 
als Kellnerin in einer angesagten Kneipe in 09_M_Stadt_AK. 14_GPS_Gö_m_GP_0 wollte hier mit seinen Freunden sein bestandenes Staatsexamen feiern, die ihm jedoch kurzfristig absagten. Zum Feiern blieb deshalb nur XX12.

Das PJ in 10_G_Stadt_D gefiel 14_GPS_Gö_m_GP_0 gut. Er begann in der Kardiologie, wo er auch einen Einblick in die Intensivmedizin bekam. Diese Fachrichtung war für ihn damals noch besonders interessant. Das zweite Tertial absolvierte er in der Chirurgie, hier war die „Ausbeute“ an den PJ-lern in seinen Augen kaum zu übertreffen. Er wurde regulär für Dienste eingeteilt, in denen er ganze Nächte lang „Haken halten“ musste. Etwas beigebracht bekommen habe er nur von einem Oberarzt. Für das letzte Tertial wechselte 14_GPS_Gö_m_GP_0 dann in die Anästhesie der 10_G_Stadt_D_Klinik_B, laut 14_GPS_Gö_m_GP_0 eine der größten Anästhesie-Abteilungen mit mehr als 120 Anästhesisten. Hier erlangte er seinen größten Lernzuwachs und erlernte auch viel „Handwerkszeug". Auch das Vertrauen in die PJ-ler schätzte er in der Abteilung sehr. Mit mehr Wissen durften man auch immer mehr Aufgaben selbstständig erledigen. Zusätzlich hatte man immer einen Oberarzt im Hintergrund für Fragen und Probleme.

Nach dem PJ und den guten Erfahrungen in der Anästhesie wollte 14_GPS_Gö_m_GP_0 eigentlich für das AiP in der Abteilung bleiben. Da er sich aber nicht in der Rolle eines Wissenschaftlers, sondern eher als „Arbeitsbiene" sah, wurde ihm kein Job angeboten. Ein weiteres Stellenangebot bekam er von der Anästhesie der 10_G_Stadt_D_Klinik_D. Diese stellten inm aber lediglich kurze Standard-OPs in Aussicht, was nicht seinen Vorstellungen entsprach. Daraufhin entschied er sich für sein AiP nach 09_M_Stadt_AG zu gehen. Bei 09_M_Stadt_AG_Klinik_A handelte es sich um ein sehr kleines Haus mit nur drei Fachabteilungen. Gelockt hatte ihn $z u$ jener Zeit die übertarifliche Bezahlung. In 09_M_Stadt_AG_Klinik_A hatte eine ganze Schar junger AiPler zur gleichen Zeit in der Inneren-Abteilung angefangen. Dies machte das Arbeitsklima extrem reizvoll. Sie erarbeiteten sich immer mehr Eigenverantwortung. Der Chef ließ sie auch gewähren. Er sah sich eher in der Rolle des ärztlichen Direktors und hatte nicht mehr viel für die Patientenversorgung übrig. 14_GPS_Gö_m_GP_0 begegnete in diesem Zuge zum ersten Mal dem „Bürokratiemonster". Er stellte beim Schreiben der Briefe fest, in der Arbeitswelt angekommen zu sein. Die Rolle des Lehrlings war nun endgültig vorbei. 
In 09_M_Stadt_AG blieb 14_GPS_Gö_m_GP_0 für insgesamt 3 Jahre in der InnerenAbteilung und 1,5 Jahre in der Chirurgie. Während dieser Zeit machte er extrem viele Nachtdienste, in denen er als Notarzt tätig war. Diese Dienste brachten zum einen die alte Leidenschaft aus dem Rettungsdienst zurück, zum anderen zerrten sie jedoch an der Substanz. 5-10 Fahrten pro 24-Stundendienst waren hier nicht ungewöhnlich. Erfreulich daran war nur, dass man in der Inneren-Abteilung nach einem Nachtdienst nicht mehr an der Visite teilnehmen musste. Dies ermöglichte ihm viele Stunden auf dem Golfplatz. Bei den Chirurgen gab es diesen Komfort nicht mehr, dafür waren die Nachtdienste im Ganzen ruhiger.

Die Tätigkeit als Notarzt fehlt 14_GPS_Gö_m_GP_0 heute, obwohl er sie auf anderem Notfalllevel auch in der Allgemeinmedizin wiederfindet. Die Ungewissheit vor jedem Einsatz, aber auch das schnelle und schmucklose Arbeiten hatten ihn immer fasziniert. 14_GPS_Gö_m_GP_0 gab aber auch zu, dass er seine persönlichen Horrorszenarien, wie der Tod eines Säuglings oder auch eine komplizierte Geburt im Rettungswagen, nie erleben musste. Mitgenommen aus dieser Zeit hatte er aber insbesondere die Fähigkeit, lebensbedrohlich Kranke von anderen Kranken zu unterscheiden.

Nach seiner Zeit in 09_M_Stadt_AG entschied sich 14_GPS_Gö_m_GP_0 für eine Niederlassung. Der Ort stand insofern fest, als dass XX12 bereits in 09_M_Stadt_AK als Lehrerin verbeamtet war und somit in ihrer Flexibilität sehr eingeschränkt war. Somit ließ 14_GPS_Gö_m_GP_0 sich als Assistent in einer Hausarztpraxis in einem Nachbarort von 09_M_Stadt_AK nieder. Als dann jedoch XX12 mit ihrem zweiten Sohn schwanger wurde, sehnte sich 14_GPS_Gö_m_GP_0 nach mehr Beständigkeit in seinem Berufsleben. Somit antwortete er auf die Stellenanzeige seines jetzigen Partners im örtlichen Tageblatt. Nach einem halben Jahr als Assistent in der Praxis und einem weiteren halben Jahr Vertretung des Vorgängers, stieg 14_GPS_Gö_m_GP_0 in die Praxis ein. Da XX12 und 14_GPS_Gö_m_GP_0 parallel mit dem Hausbau begonnen hatten, entschied sich 14_GPS_Gö_m_GP_0 für einen Arbeitsvertrag mit „ansteigender Gewinnverteilung“. So fielen für die Praxis keine zusätzlichen Kredite an. Im kommenden Januar wird 14_GPS_Gö_m_GP_0 gleichwertiger Partner in der Praxis sein und freut sich schon jetzt auf die ersten Investitionen, gerade in der Praxisgestaltung.

Mit der Wahl des Praxisstandortes ist 14_GPS_Gö_m_GP_0 immer sehr zufrieden gewesen, er kann so mittags und auch abends schnell zu seiner Familie. Seine Familie ist 
14_GPS_Gö_m_GP_0 sehr wichtig gewesen. Seine beiden Jungs, so findet er, sind ihm wirklich gut gelungen. Auch schätzt er die Nähe zu seinen Eltern und Schwiegereltern, die beide regelmäßig die Aufgaben des Babysitters übernehmen, so dass auch XX12 weiterhin als Lehrerin arbeiten kann. Von ihr kam in diesem Zusammenhang auch der Einwurf, dass sie nicht die richtige Frau sei, um zu Hause zu bleiben.

Wie bereits oben erwähnt, war 14_GPS_Gö_m_GP_0 am Tag der Interviewführung besonders zufrieden, weil er ab Anfang des nächsten Jahres zu 50 \% Teilhaber an der Praxis sein wird. In Bezug auf die Patienten erwähnte er, dass auch eine Sterbebegleitung ihn zufrieden macht. Gerade die Gewissheit, alles für den Patienten bis zum Tode getan zu haben, ist für ihn besonders wichtig. Während 14_GPS_Gö_m_GP_0 jedoch über die Sterbebegleitung nachdachte, erinnerte er sich an den Tod einer Freundin. Hier empfand er die Begleitung als sehr belastend und wünschte sich, eine solche Situation nicht wieder erleben zu müssen. Auch die Tatsache, aus einem vollen Wartezimmer den Pateinten mit dem Herzinfarkt richtig diagnostizieren zu können, erfüllt 14_GPS_Gö_m_GP_0 mit Zufriedenheit.

Unzufrieden fühlte er sich lediglich im Hinblick auf das „Bürokratiemonster“. Regressforderungen, Budgetierungen und das mangelnde Vertrauen des Gesundheitssystems in die Entscheidungsfähigkeit des Arztes ließen 14_GPS_Gö_m_GP_0 verzweifeln. Dies waren auch seine Punkte, die er den Politikern mit auf den Weg geben wollte. In 14_GPS_Gö_m_GP_0‘s Augen ist das System, so wie es momentan sei, „schwachsinnig“. Er wünschte sich eine höhere Kostentransparenz sowohl für Patienten als auch Ärzte. Langfristig, so glaubte er, würde Deutschland auch nicht an einer Bürgerversicherung mit privaten Zusatzzahlungen vorbeikommen, auch wenn das gerade für Sozialschwache Ungerechtigkeit bedeuten würde. Ein anderes System, das die massiven Kosten langfristig abdecken würde, konnte 14_GPS_Gö_m_GP_0 sich nicht vorstellen. Ärzte, so fand er, meckern jedoch insgesamt auf sehr hohem Niveau über die Lage in Deutschland. 14_GPS_Gö_m_GP_0 war zufrieden mit seinem Einkommen und hatte gerade als „Landarzt“ keine Bedenken, in die Arbeitslosigkeit zu geraten. Für ihn sei das Leben auf dem Land in einer Region wie 09_M_Stadt_AK, mit so geringen Arbeitslosenzahlen, seine Erfüllung.

\section{Erste Ideen für die Auswertung}

- Kindheit in 09_M_Stadt_AK 
- Entscheidung des Lebenspartners

- Frage nach Kinderbetreuung 


\subsection{Anlage 7: offenes Kodieren in NVivo9}

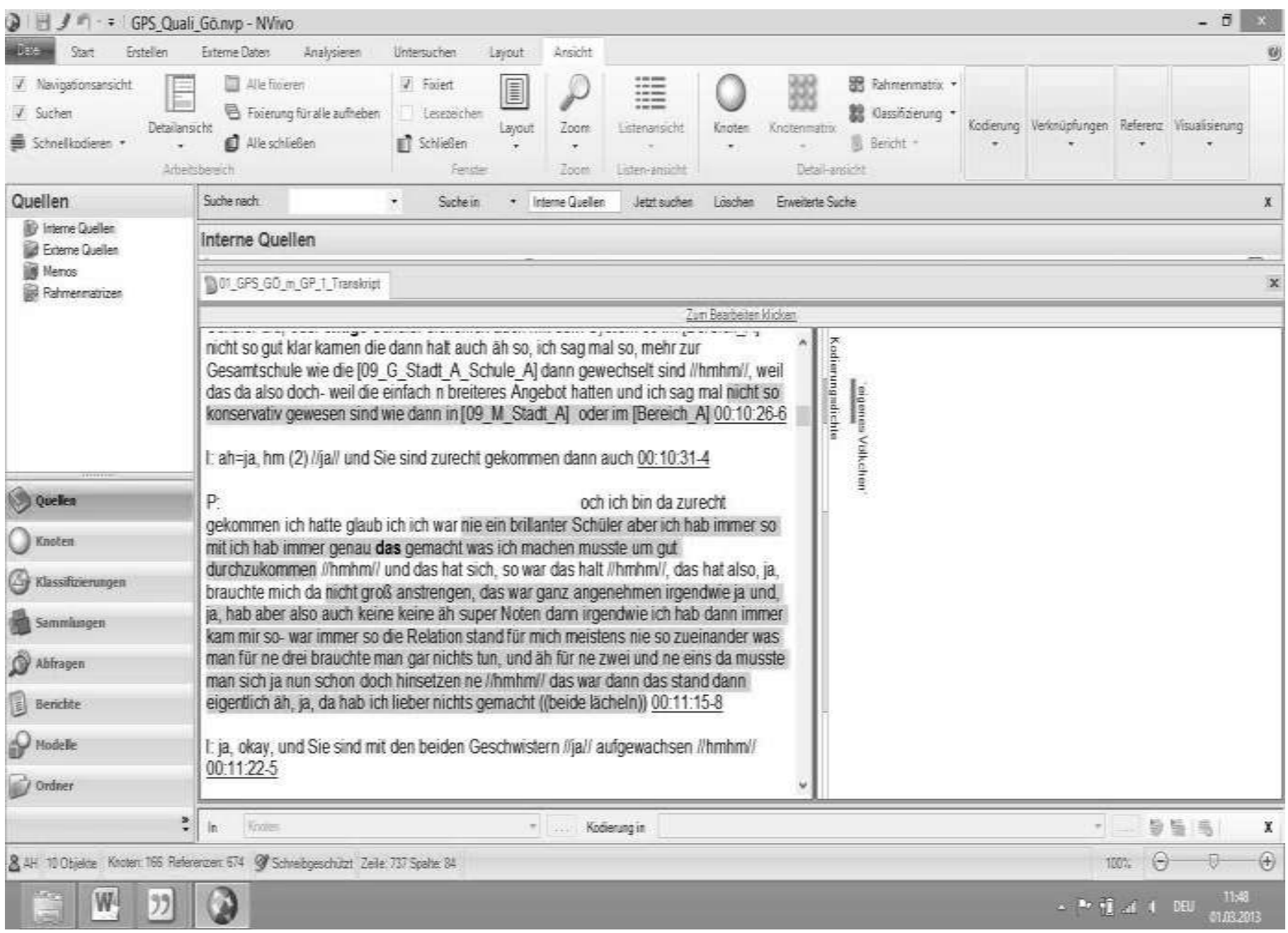




\subsection{Anlage 8: Memo entstanden während des Kodierens}

GPS Niedersachsen- Gedanken beim Kodieren

Memo geschrieben am:

13.12.2012

Uhrzeit:

16:30 Uhr

Ort:

Göttingen

Von:

Annika Heubrock

Interview:

10_GPS_Gö_EP_0

Text:

Insgesamt hatte ich den Eindruck, dass 10_GPS_Gö_EP_0 generell eher kurz angebunden war. Sie schweifte in ihren Aussagen kein bisschen aus und beschränkte sich sehr auf Fakten. 10_GPS_Gö_EP_0 ist mit der Medizin viele Kompromisse eingegangen. Sie hatte Medizin nur als Ausweichfach in Erwägung gezogen. Eigentlich wollte sie Tiermedizin studieren. Aufgrund der Gegebenheiten im Ort und der Einstellung ihrer Oma hatte sie sich aber letztendlich dagegen entschieden. Hier wurde bereits ihre enge Familien- und Ortsanbindung deutlich!

„Eigentlich wollte ich ja Tiermedizin machen. (...) Dann sagte meine Oma damals zu mir: , mein Kind das finde ich ganz toll, dass du das machen willst, so mit Kleintieren. Dann kannst du dich auch immer um die Katze kümmern und die Vögel. Aber Kühe, Schweine, Pferde', sagte meine Oma, ,nein mein Kind, das machen nur Männer. So ist es halt auf dem Dorf.' Auf dem Dorf damals gab's keine Tierärztin. Jetzt sieht das ganz anders aus, aber damals gab's das halt nicht und dann hab ich gesagt: ,nee, dann mach ich keine Tiermedizin, dann mach ich einfach Humanmedizin'."

Der zweite Kompromiss in Bezug auf die Medizin war die Entscheidung für die Allgemeinmedizin. Eigentlich fand 10_GPS_Gö_W_EP_0 die Allgemeinmedizin immer extrem langweilig.

„Allgemeinmedizin hätte ich mir nie vorstellen können. (...) Zu meiner Zeit gab's das schon als Fach im Studium. Da war dann auch nur ein Kollege aus meiner näheren Umgebung daein älterer Hausarzt, der diese Kurse geleitet hat. Fand ich ganz schrecklich, hat mich überhaupt nicht angesprochen." 
Als sie jedoch keine Stelle in ihrem eigentlichen Wunschfach Pädiatrie bekam, entschloss 10_GPS_Gö_W_EP_10 sich doch in die Allgemeinmedizin zu gehen.

„Dann habe ich mich entschlossen: Pädiatrie kriegst du jetzt keine Stelle mehr, also machst du Allgemeinmedizin. Dann kannst du immer mal ein paar Kinder mitbehandeln, dann wirfst du es nicht ganz weg."

Trotzdem scheint sie mit dieser Entscheidung im Nachhinein zufrieden zu sein.

„Also retrospektiv war das die absolut richtige Entscheidung. Der Pädiater ist halt der Hausarzt der Kinder, aber es ist ruhiger bei mir im Wartezimmer."

Die Tatsache, dass 10_GPS_Gö_w_EP_0's Oma bereits bei der Entscheidung zum Studium mit Einfluss nehmen durfte zeigt, dass 10_GPS_Gö_W_EP_0 sehr viel Wert auf ihre Familie und einen familiären Zusammenhalt legt. So geht sie zum Studium auch nicht von zu Hause weg

„I: Hab ich das dann richtig verstanden, dass sie die ganze Zeit eigentlich im Heimatdorf gewohnt haben?

P: Ja, immer."

und bezieht auch bei der Kindererziehung ihre Familie mit ein.

„Dadurch, dass ich eigentlich eine total intakte Familie zu dem Zeitpunkt hatte, hat das eigentlich sehr gut funktioniert. Also meine Oma war zu dem Zeitpunkt noch da und die hat sich ganz viel um die beiden gekümmert. Meine Mutter selber ist noch arbeiten gegangen und mein Vater auch."

Eigentlich wollte 10_GPS_Gö_w_EP_0 mit ihrem Mann ins Ausland auswandern, doch auch hier nimmt ihre starke familiäre Bindung Einfluss. So verwerfen sie ihre Pläne aufgrund einer Erkrankung der Großmutter.

„Also wir hatten zwischenzeitlich auch mal geliebäugelt auszuwandern nach [61] oder [26]. Mein Mann ist Ingenieur und hätte sich dorthin versetzen lassen können. Damals gab's dann auch die Angebote sich dann dort als Arzt auch niederzulassen und da haben wir wirklich mit geliebäugelt. Aber es ist daran gescheitert - die Kinder waren zu dem Zeitpunkt noch relativ klein - , dass man niemanden für die Kinder hatte. Und als die Kinder dann größer waren ging 
es meine Oma nicht gut. Die war halt sehr krank und wir haben gesagt, wir können jetzt nicht gehen - wegen der Kinder, weil das das Einzige ist, was sie halt noch aufrechterhalten hat. Was sie halt gefreut hat, das waren so die ersten Urenkel und das war der Grund, warum wir geblieben sind."

Auch bei der Praxisstandortwahl nimmt die Familie Einfluss.

„Der hat mir diese Praxisräume hier vorgeschlagen. Also der wusste, dass die hier frei werden. Gut war, dass das letztendlich in der Nähe von meinem Wohnort war."

Über dem Wunsch, nah an der Praxis zu wohnen, steht aber noch der Wunsch, ein ruhiges Familienleben zu haben.

„Das war mir ganz wichtig, ich wollte das nicht so haben wie bei uns auf dem Dorf, dass ich am Wochenende die Patienten vor der Tür stehen habe oder nachts um zehn. Sondern ich wollte es schon getrennt haben. Das war mir halt ganz wichtig, dass meine Kinder sich auch irgendwie frei entwickeln können und nicht immer so: ,guck mal, da läuft die Tochter vom Arzt rum und wie die aussieht.' Wenn man dann irgendwo auf öffentlichen Feiern stehtSchützenfest- und mal ein Bier trinkt ja: , ah haste gesehen' (verstellt ihre Stimme). Das wollte ich nicht und das war mir einfach wichtig, dass ich wirklich auch meine Ruhepausen habe. Ich bin schon für einige Patienten, denen es nicht gut geht, auch hier telefonisch erreichbar, aber halt nicht richtig im Kontakt. Freunde und Bekannte kommen trotzdem aber, dass ist nicht die Mehrzahl. Das war mir wichtig, auch bei dem Aussuchen des Praxisstandortes war mir das sehr wichtig."

Sogar die Mutter ist im Praxisprojekt involviert und hilft direkt bei der Abrechnung.

„Meine Mutter ist MTA und die arbeitet immer noch mit und bringt natürlich ganz viel Wissen mit."

Auffällig ist, dass 10_GPS_Gö_w_EP_0 viele Dinge übernommen hat, die sie früher an ihrem eigenen Chef kritisiert hat

Kritik an Patientennähe:

„Also mein Chef war immer sehr offen und hat auch von seinen privaten Sachen erzählt, wo ich immer dachte: boah, was geht den das eigentlich an? Also ich war da immer reservierter, 
zumindest in der Zeit dort. Jetzt, wo ich meine eigene Praxis habe, ist es genauso. Also man hat wirklich so eine, in Anführungsstrichen, "familiäre Bindung" zum Patienten. Die erzählen von den familiären Sachen auch und fragen auch nach. Damals hätte ich nie so offen geantwortet, aber jetzt hier in meiner eignen Praxis finde ich das auch völlig natürlich."

\section{Kritik an Hausbesuchen:}

„Die Zeit da war sehr geprägt von Hausbesuchen. Fand ich total ätzend. Gehört zum Allgemeinmediziner dazu, kostet aber unwahrscheinlich viel Zeit. Also im Grunde genommen sind die Hausbesuche nett und es ist schön, dass man weiß wie die Patienten zu Hause leben, aber dass es so viel Zeit in Anspruch nimmt, neben der normalen Patientenversorgung, das nervt. Ich bin da so ein bisschen gespalten. Da hatte ich halt unwahrscheinlich viele Hausbesuche, weil er halt natürlich keine gemacht hat. Vielleicht deshalb auch so ein bisschen diese Abneigung. Also ich mache hier auch Hausbesuche, aber das war da nicht so prickelnd."

Zudem ist 10_GPS_Gö_W_EP_0 sehr auf Sicherheit bedacht. So testet sie den Krankenhausalltag zuvor mit einer Ausbildung und hat auch bereits eine berufliche Alternative, falls die Praxistätigkeit nicht erfolgreich verläuft.

„Das war eigentlich vorher schon klar! Nur ich wollte wirklich wissen, ob mir das im Krankenhaus liegt. Wenn ich dann gemerkt hätte, gut die Arbeit im Krankenhaus oder das System Krankenhaus passt mir nicht, dann hätte ich mir sicherlich was anderes überlegt. Aber das hat einfach auch nochmal bestätigt."

„Hatte aber immer auch noch parallel sehr guten Kontakt zum [09_M_Stad_Q_Klinik_A].Die damalige Funktionsoberärztin ist gegangen und die Stelle hatten sie mir angeboten und ich hab immer gesagt: ja wartet mal, wenn es nicht klappt melde ich mich. Gott sei Dank hat es geklappt. Aber ich hab mich immer doch nach mehreren Seiten auch abgesichert. Das war mir auch wichtig. Wenn es dann in die Hose geht, ist es ja nicht schlimm. Dann hat man eben auch noch Alternativen."

Interessante Punkte für die Auswertung

\section{- Kindererziehung Studium}

- Praxisstandort Familienfreundlichkeit

- Stellenmangel führt zur Niederlassung $\rightarrow$ Sicherheitsaspekt 


\subsection{Anlage 9: Kategorie-Memo: Klinikfrust}

\section{Kategorie-Memo: Negativmotivation „Klinikfrust“}

In sehr vielen Interviews erscheint der „Klinikfrust“ als Hauptmotivation oder zumindest als einer der zuletzt ausschlaggebenden Faktoren für eine hausärztliche Niederlassung.

In erster Linie nehmen die Hausärztinnen und Hausärzte (HÄ) hier Bezug auf die Dienstbelastung im Krankenhaus.

01_GPS_GÖ_m_GP_1; Seite 2, Zeile 20-28:

„In der Folge hab ich dann anderthalb Jahre in der Weiterbildung als Weiterbildungsassistent hier in der Praxis meines Vaters gearbeitet, um auch den Einblick zu bekommen. Das hat mir sehr gut gefallen, insbesondere dass die Dienste doch ganz anders als in der Inneren waren. Das kennen Sie wahrscheinlich, das war dann zum Schluss, wo ich wirklich sagen musste: Mensch das verdirbt also die Freude am Job. Dass man für die Dienste, die man nachts tätigt, hinterher noch weniger Geld bekommt beziehungsweise Abzüge hat, das ist eigentlich schon schade."

01_GPS_GÖ_m_GP_1; Seite 12, Zeile 24-27:

„Ich sag mal so: in der Zeit war einfach vordergründig, dass ich zum Schluss einfach auf die Dienste keine Lust mehr hatte. Muss ich ehrlich sagen. Das war einfach nur noch belastend und einfach nervend'."

Es werden sehr starke Begriffe wie „belastend“ und „nervend“ verwendet und als Ergebnis der Verlust der Freude am Beruf beschrieben.

06_GPS_Gö_m_GP_0; Seite 54, Zeile 704:

„War natürlich viel, wir hatten damals elf Dienste im Monat."

03_GPS_GÖ_w_GP_1; Seite 3, Zeile 40-42:

„Ich merkte dann im Krankenhaus, das ist es irgendwie nicht so auf die Dauer. Da war nicht die Perspektive; die ständigen Dienste und Notarztdienste und was man da alles so machen muss." 
03_GPS_GÖ_W_GP_1; Seite 4, Zeile 42-45:

„Jede dritte, vierte, also das ist jetzt vielleicht $n$ bisschen übertrieben aber vom Gefühl her waren da viel mehr Nachtdienste. Es waren nicht so viele, wie ich jetzt vielleicht retrospektiv denke, aber man war einfach viel mehr weg."

Hier wird ebenfalls angesprochen, dass die Kollegin auf Grund der Dienste seltener zu Hause war, was ebenfalls eine Belastung für die Familie, insbesondere die jüngeren Kinder gewesen sei.

Aber auch eine nicht dementsprechende Konstitution wird in Eigenerkenntnis beschrieben: 05_GPS_GÖ_w_EP_1; Seite 10, Zeile 422-425:

„Wie gesagt, die Dienste, das war das Problem. Also was ich absolut nicht gut abkann, wenn ich nachts ständig raus muss. Das erwähnte ich vorhin schon mal."

Zu der Dienstbelastung wird jedoch auch ein insgesamt höherer Druck und damit einhergehender Zeitverlust für die persönliche Zeit mit den Patientinnen und Patienten bemängelt mit der gleichen Konsequenz des Verlusts der Berufsfreude.

01_GPS_GÖ_m_GP_1; Seite 7, Zeile 11-14:

„Dann natürlich der ganze Druck. Ich sag mal die ganzen Arztbriefe, alles muss schneller gehen, noch tausend Sachen nebenbei und zigtausend Dienste, das war dann einfach ne Sache, wo ich wirklich zum Schluss keine Lust mehr hatte. Das hat's mir wirklich verdorben."

Letztlich wird die Klinik-Situation auch als chaotisch und zunehmend verschlechternd beschrieben.

03_GPS_GÖ_W_GP_1; Seite 16, Zeile 18-22:

„Wenn ich mir jetzt die Situation angucke, wie das in dem Krankenhaus geworden ist, bin ich heilfroh, dass ich da nicht auf irgendwelche Überlegungen eingegangen bin. Da herrscht weiterhin das Chaos. Ich glaub, das hat sich einfach gut gefügt."

04_GPS_GÖ_W_GP_0; Seite 8, Zeile 312-313: 
04_GPS_GÖ_W_GP_0: Seite 8, Zeile 313-314:

„Es kamen immer mehr völlig unfähige Kollegen, deren Arbeit man mitmachen musste, dazu, was immer weniger Spaß gemacht hat."

04_GPS_GÖ_W_GP_0; Seite 8, Zeile 315-317:

„Es wurde angedroht, dass der Krankenhausträger privatisiert. Es wird [09_G_Stadt_C_Klinik_D] oder ist [09_G_Stadt_C_Klinik_D] geworden mittlerweile. Da bin ich mittlerweile auch sehr froh, dass ich da nicht mehr bin."

Mit zunehmender Eigenständigkeit in und nach der Ausbildung werden zudem zunehmend die Anordnungen der Vorgesetzten als nicht nachvollziehbar beschrieben.

04_GPS_GÖ_W_GP_0; Z:330-338

„Es ist wunderbar sein eigener Herr zu sein, nicht mehr auf irgendwelche merkwürdigen Ratschläge von Ober- oder Chefärzten zu hören. Außerdem haben wir einen neuen Chefarzt gekriegt. Es waren zwei Chefärzte dort, der eine Chefarzt hat nicht nur mir nicht gefallen, sondern allen anderen auch nicht. Ich hab, glaube ich, so ein bisschen den Anfang gemacht: es sind dreizehn andere Fachärzte gegangen innerhalb anderthalb Jahren, nicht umsonst. Da merkt man einfach, dass das Klima in diesem Krankenhaus nicht mehr so ist."

Eine Kollegin beschreibt zudem eine vollkommene Enttäuschung vom Krankenhausbetrieb auf verschiedenen Ebenen. So wurde sie in ihrer Zeit als ÄiP von Pflegekräften gemobbt, diese für dieses offensichtliche und erkannte Fehlverhalten nicht von den Chefs zur Rechenschaft gezogen und darüber hinaus habe keine Anleitung, keine Weiterbildung stattgefunden.

07_GPS_GÖ_w_MVZ_0; Seite 20, Zeile 847-851:

„Da waren so zwei, drei, die konnten mich wohl überhaupt nicht ab. Die eine hat sich dann irgendwann hingestellt und während einer Visite, wo ich mit dem Assistenzarzt da rumging, gesagt sie dürften keine Anordnungen von mir mehr annehmen und so. Also richtig unwahre Behauptungen in die Welt gestellt." 
07_GPS_GÖ_w_MVZ_0; Seite 20, Zeile 864-867:

„Das war ganz schlimm und dann stellte sich raus, dass das von dem auch nicht kam und das hatte aber leider keine Konsequenzen für die, die da solche Behauptungen taten. Das fand ich auch ganz schön schlimm."

07_GPS_GÖ_w_MVZ_0; Seite 20, Zeile 876-886:

„Das Mobbing, das war ja noch viel viel schlimmer. Das andere, was ich meinte, war dass ich fand, dass die Oberärzte und Chefärzte einen nicht besonders gut angeleitet haben. Also vielleicht schon eher mal die Assistenzärzte, aber eigentlich ist es auch Aufgabe der Oberärzte. Also, da kam nicht viel. Die machten halt ihre Arbeit, aber (...) dass man uns da irgendwie mal was beigebracht hätte oder sich mal nen bisschen mehr um uns gekümmert hätte, also ich hab das so nicht besonders erlebt."

Letztlich wird ein Zustand der vollkommenen Unzufriedenheit während der Arbeit in einer Klinik beschrieben, welche sich im Vergleich zu den erfahrenen Zuständen in einer eigenen allgemeinmedizinischen Praxis noch deutlicher darstellen, hierin jedoch auch ihre Auflösung finden.

04_GPS_GÖ_w_GP_0; Seite 10, Zeile 400-402:

„Vorher habe ich mich furchtbar oft geärgert: über meine Arbeit, über meine Chefs, über meine Kollegen, über was weiß ich, über alles Mögliche."

01_GPS_GÖ_m_GP_1; Seite 12, Zeile 37 -Seite 13, Zeile 8:

„Da stand für mich fest, in der Klinik geht's auf keinen Fall weiter. Hatte das ja so geplant, dass ich dann hier auch in die Praxis gehe und das hat dann auch wirklich Spaß gemacht. Da stand dann für mich ehrlich gesagt auch fest, einfach als ich gesehen habe, dass das ein ganz anderes Arbeiten ist und einfach wirklich Spaß macht und auch erfüllend sein kann. Das war so die Situation, würde ich sagen. Also auf der einen Seite die Negativerfahrung, in der Klinik, nicht von der Arbeit an sich her, sondern einfach von der Arbeitsbelastung her. Das hat zum Schluss einfach keinen Spaß mehr gemacht, wenn Sie für nix mehr Zeit haben. Sie können sich für keinen Patienten Zeit nehmen und alle sind nur am Anrufen und zerren hier hin und da hin: können Sie noch mal das machen und noch mal das machen? Das ist dann kein Spaß. Dann also hier die Erfahrung, dass man auch mal sagen konnte, okay, man hat mal die Zeit, 
sich noch mal mit einem Patienten hinzusetzen und mal zwanzig Minuten zu reden, wenn da gerade wirklich Bedarf ist, dass irgendwer gestorben ist oder schwierige Entscheidungen anstehen.(...) Zeit, die man eigentlich so auch als menschlich notwendig ansieht, dass man die sich auch nehmen kann, dass habe ich dann doch hier in der Praxis zu schätzen gelernt." 
11.10 Anlage 10: Internetquellen

http://www.bundesaerztekammer.de/page.asp?his=0.2.23.2450.2451.2459

Beschlussprotokoll 105. Dt. Ärztetag 2002: Zu Punkt I der Tagesordnung: Gesundheits-, Sozial- und ärztliche Berufspolitik

\section{I, 8 : Dem deutschen Gesundheitswesen gehen die Ärzte aus}

Auf Antrag des Vorstandes der Bundesärztekammer (Drucksache l-3) fasst der 105. Deutsche Ärztetag folgende Entschließung:

Die von Bundesärztekammer und Kassenärztlicher Bundesvereinigung vorgelegte Analyse zur Altersstruktur und Arztzahlentwicklung zeigt auf, dass sich der Arbeitsmarkt für die deutschen Ärztinnen und Ärzte im Umbruch befindet: Die deutsche Ärzteschaft hat gravierende Nachwuchsprobleme. Hieraus entstehen schon jetzt bundesweit Versorgungsengpässe vor allem in der Allgemeinmedizin, aber auch in anderen Fachbereichen. Die anwachsende Zahl von Stellenausschreibungen und die festgestelte Zunahme des Durchschnittsalters betrifft insbesondere die ostdeutschen Länder.

Durch überbordende Reglementierungen und Bürokratisierung der ärztlichen Berufsausübung und einer budgetbedingten Arbeitsūberlastung ist der Arztberuf in Klinik und Praxis offensichtich zunehmend unattraktiv geworden. Die Situation stellt sich folgendermaßen dar

- Der "Verlust" an Medizinstudenten und -studentinnen im Studienverlauf liegt zwischen 30 bis 40 Prozent. In dieser unvertretbar hohen Quote befinden sich Studienabbrecher und Studienwechsler sowie eine etwa gleich große Zahl von Studienabsolventen, die jedoch nicht in die AIP-Phase eintreten und offensichtlich in alternative Berufsfelder gehen.

- Die Zahl der Allgemeinmediziner und Praktischen Ärzte hat in den letzten Jahren kontinuierlich abgenommen; in den neuen Bundesländern werden zudem in den nächsten fünf bis zehn Jahren ca. 40 Prozent Hausärzte die Altersgrenze erreichen und somit aus dem Berufsleben ausscheiden.

- Insbesondere in den neuen Bundesländern stellt sich die Altersproblematik auch in anderen Fachgebieten besonders dramatisch dar: es fehit der Nachwuchs einerseits - andererseits ist die Versorgung durch ein überproportionales Ausscheiden von Rentnern und Ruheständlern gefährdet.

- Vor allem in den ländlichen Regionen wirkt sich der Ärztemangel schon heute aus und greift bereits auf Ballungsgebiete über:

- Auf Grund der schlechten Arbeitsbedingungen wählen immer mehr Ärzte den frühestmöglichen Zeitpunkt für ihre Pensionierung oder scheiden ganz aus dem Arztberuf aus.

- Obwohl fûr 2001 die Gesamtstatistik noch eine gering positive Steigerungsrate von 1,6 Prozent aufweist, ist die Rate an berufstätigen Ärztinnen und Ärzten in einigen Landesärztekammern bereits jetzt negativ; ein warnendes Anzeichen für einen sich auf absehbare Zeit generell abzeichnenden Ärzternangel ist der deutliche Rückgang an stationär tätigen Ärzten und Ärztinnen in fünf Landesärztekammern.

- Die gegenwärtig bestehenden regionale Versorgungsengpšsse werden sich vor dem Hintergrund erhöhter Behandlungserfordernisse infolge Langlebigkeit, Multimorbidität und medizinischen Fortschritts erheblich verschärfen.

Der Deutsche Ärztetag fordert daher eine konzertierte Aktion von ärzticher Selbstverwaltung, Politik und Krankenkassen, um die fatale Entwicklung zu stoppen und möglichst umzukehren. Durch Abbau staatlicher Reglementierungen sowie durch angemessene Arbeits- und Vergütungsbedingungen am Krankenhaus und in frejer Praxis muss dem Arztberuf wieder der Stellenwert eingeräumt werden, den er im Ansehen der Bevökerung nach wie vor hat.

Es ist an der Zeit, die Diffamierung des Arztberufes einzustellen.Der Arztberuf muss wieder attraktiv werden.

Junge Ärztinnen und Ärzte können verstärkt motiviert werden, wieder in die praktische Medizin zu gehen.

Dazu sind folgende Maßnahmen geeignet:

- Umsetzung der novellierten Approbationsordnung mit praxisnahem Medizinstudium an den Medizinischen Fakultäten aller Bundesländer

- Änderung der Bundesärzteordnung zwecks Abschaffung des AiP's als überfälige Maßnahme

- Verbesserung der ärztlichen Weiterbildung in Klinik und Praxis und Bereitstellung der hierfür notwendigen Mittel

- Schaffung einer einheitlichen Hausarzt-Qualifikation und weiterer Anreize für die

Weiterbildung in der Allgemeinmedizin

- Anerkennung des Bereitschaftsdienstes als volle Arbeitszeit entsprechend des Urteils des Europaischen Gerichtshofes

- Verbesserung der Rahmenbedingungen für die Vereinbarkeit von Beruf und Familie

- Reduzierung und Bezahlung von Überstunden

- Aufhebung der Ost-West-Finanzierungsłücke und gleicher Lohn für gleiche Arbeit

Gelingt es nicht, mit diesen und weiteren Maßnahmen die fatale Entwicklung umzukehren, wird es zu unübersehbaren Folgewirkungen mit einem Versorgungsnotstand in ganz Deutschland kommen und die Patienten werden unter dem Mangel an medizinischer Betreuung und ärztlicher Zuwendung zu leiden haben. 
Ergebnisse der Ärztestatistik zum 31. Dezember 2012 Kein Widerspruch - Ärztemangel trotz moderat steigender Arztzahlen

Wurde das Problem des Ärztemangels lange Zeit von der Politik kleingeredet oder sogar geleugnet, wird heute kaum mehr bestritten, dass in vielen Kommunen der wohnorthahe Zugang zu arztlichen Leistungen nicht mehr gegeben oder zumindest eingeschränkt ist. In vielen Regionen fehtt es an niedergelassenen Haus- und Fachärzten aber auch in den Krankenhausern sind bundesweit mehr als 6000 Arztstellen unbesetzt.

Aber wie passen moderat steigende Arztzahlen, wie sie auch die aktuelle Ärztestatistik von Bundesäztekammer und Kassenärztlicher Bundesvereinigung ausweist, und zunehmender Ärztemangel zusammen?

Die Gründe hierfür sind vielfältig. Zum einen bedingt der medizinische Fortschritt mehr Leistungen und einen Anstieg der Behandlungszahl, weil früher nicht bekannte oder mögliche Eingriffe, Untersuchungen und Therapiemethoden mit zunehmendem Fortschritt durchführbar geworden sind. Dies erfordert ebenso mehr Personal, wie die durch die wissenschaftliche Entwicklung bedingte zunehmende Spezialisierung der Medizin. Vermehrten Personalbedarf ziehen auch Vorgaben der Politik, beziehungsweise des Gemeinsamen Bundesausschusses hinsichtich neuer Qualifikationsanforderungen nach sich. Als Beispiele seien hier das Gendiagnostikgesetz und das infektionsschutzgesetz genannt.

Zugleich ziehen auch die demografischen Veränderungen erhebliche Auswirkungen nach sich. So ist der Anteil der über 59-Jährigen an der Gesantbevölkerung von 1991 bis heute um ein Fünftel gestiegen. In folgedessen hat die Behandlungsintensität erheblich zugenommen. Hinzu komnt, dass die demografische Entwicklung langst auch die Ärzteschaft selbst erfasst hat, wie die aktuelle Ärztestatistik zeigt. Das Durchschnittsalter der Ärztinnen und Ärzte in Klinik und Praxis nimnt stetig zu. Es ist schwierig, Nach folger zu finden, wenn sich an den aktuellen Bedingungen der Berufsausübung nicht schnell etwas ändert.

Ein weiterer Grund für den Arztstundenmangel: Die nachwachsende Ärztegeneration hat eine andere Sicht auf die sogenannte Work-Life-Balance. Sie misst dem Familienleben einen höheren Stellenwert zu und entscheidet sich häufiger als früher für Teilzeitarbeit. Hinzu kommt, dass sich die Zahi der Ärztinnen und Ärzte ohne arrztliche Tätigkeit - soweit sie bei den Landesärztekammern registriert sind - wie in den Vorjahren erhöht hat. Die Zahl der Abwanderung aus Deutschland verharrt mit 2.242 Ärztinnen und Ärzten weiter auf einem hohen Niveau.

Wirksam begegnen lāsst sich dieser Entwicklung nur, wenn die Arbeit von Ärztinnen und Ärzten wieder attraktiver gestaltet wird, so dass mehr junge Berufsanfänger in der kurativen Medizin tätig werden. Eine Gesellschaft des langen Lebens braucht Ärztinnen und Ärzte in Klinik und Praxis und nicht in anderen Berufsfeldern.

Alle Diagramme und Tabellen [PDF

- Die ärztliche Versorqung in der Bundesrepublik Deutschland Ergebnisse der Ärztestatistik zum 31.12.2012

- Gesamtzahl der Ärzte

Im Jahre 2012 ist die Gesantzahl der bei den Landesärztekammern gemeideten Ārztinne und Ärzte auf 459.021 gestiegen. Dies sind 2,1 Prozent mehr als im Jahre 2011; damit liegt die Zuwachsrate im Trend der letzten Jahre, aber etwas niedriger als im Vorjahr.

- Berufstätige Ärzte

Ohne die 110.326 nicht ärztlich Tätigen waren im Jahre 2012 im Bundesgebiet 348.695 Ärztinnen und Ärzte arrtlich tätio, dies waren 6.632 mehr als im Voriahr. Die Zuwachsrate betrug damit 1,9 Prozent.

- Im Krankenhaus tätige Ärzte Der Anteil der im Krankenhaus tätigen Ärztinnen und Ärzte ist bezogen auf alle ärztlich Tätigen weiter angestiegen auf nun 50,1 Prozent (Vorjahr: 49,7 Prozent). Damit sind meht als die Hälfte aller ärztlich Tätigen in einem Krankenhaus beschäftiot:

- Ambulant tätiqe Ärzte

Die Zahl der ambulant tätigen Ärztinnen und Ärzte ist im Jahre 2012 um 0,8 Prozent Die Zahil der ambulant tátigen Arztinnen und Ärzte ist im Jahre 2012 um 0,8 Prozent angestiegen, was 1.203 Arztinne

- In Behörden/Körperschaften und in sonstigen Bereichen tätige Ärzte Fasst man die Tätigkeitsfelder bei Behörden/Körperschaften und in sonstigen Bereichen. zusammen, so waren dort mit 29.808 rund 1,5 Prozent mehr Ärztinnen und Ärzte tätig als im Vorjahr

- Ärzte ohne ärztliche Tätiqkeit

Die Zahl der Ärztinnen und Ärzte ohne ärztiche Tätigkeit - soweit sie bei den Landesärztekammern registriert sind - hat sich 2012, wie die Jahre zuvor, erhöht Die Steigerungsrate betrug 2,8 Prozent. Dies entspricht 2.980 Ärztinnen und Ärzten.

- Anzahl der erteilten Anerkennungen

Im Jahre 2012 wurden 11,891 Anerkennungen von Facharztbezeichnungen

ausgesprochen. Damit ist die Zahl gegenüber den 11.548 Anerkennungen des Jahres 2011 weiter angestiegen.

- Ausländische Ärztinnen und Ärzte

Die Zahl der in Deutschland gemeldeten ausländischen Ärztinnen und Ärzte ist im Jahre 2012 um 4.193, das entspricht 14,8 Prozent, auf 32,548 gestiegen.

- Abwanderung von Ärzten ins Ausland

Selt 2005 werden bei den Ärztekammern die Daten bezüglich der Abwanderung von Arzten ins Ausland erhoben. Im Ergebnis lässt sich feststellen, dass im Jahre 2012 Ansgesamt 2.241 ursprünglich in Deutschland tätige Ärztinnen und Ärzte ins Ausland abgewandert sind, wobei der Anteil der deutschen Ärzte 66,8 Prozent beträgt.

Bookmarkservice

\section{f $\mathrm{C}$. $\mathrm{x} \mathrm{G}$}

zum Anfana der Seite

๑๑) Bundesärztekammer - letzte Änderung 08.07.2013 
http://www.degam.de/summerschool-2013.html

\section{Bewerbung}

Bewerbungen bitte per E-Mail an allgemeinmedizineunigreifowald.de oder per Post.

Ein Anmeideformular kann auf der Website der DEGAM twww.depam.de im Unterpunikt, studium und Hochschule" heruntergeladen werden.

Deses Praictr weo Gerbanerer DUACH

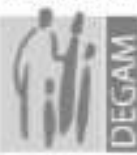

OQ DEUTSCME

HAUSARZTEVERBAND

GHA

Selelochaft der tochubulletver fur Alyemeinmediain $e_{\text {.V. }}$

\section{Ansprechpartner}

Universitätsmedizin Greifswald

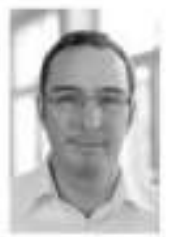

Fot. Or.med. Jean francois Chenot, Mor Abteilune Allemeinmedtain insteut fir Community Medione Univerntibarnedicin Greihwald Elemholvutralle 1-2 17487 Contwald

Telefor $03834 \$ 62228$ fax $03834 \$ 62228$ E.Mol: jhenoterningreifswaid de
Voranküindigung

3. Summerschool Allgemeinmedizin für Medizinstudierende

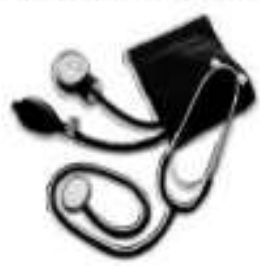

vom 28. August bis 01 . September 2013

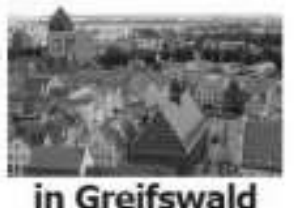

\section{Was ist eine Summerschool?}

Elne Summerschool ist ein international verbreitetes Konzept zur Nachwuchsforderure, um Studierenden Gelegenheit zu geben, sich in ein fach zu vertiefen. Die summerschool ist Teil der Nachiwuchsakademie der DEGAM. Eine Teilnahme ist auch ohne Aufnahme in die DEGAM-Nachwuchsakademie moglich.

\section{Wann findet sie statt?}

Anreise ist am Mittwoch, den 28. August 2013. Wir treffen uns ab 17:00 Uhr. Die Abrekse ist am Sonntag den 01. September 2013 margens.

\section{Wo findet sie statt?}

Die summerschool Algemeinmedizin findet im Universitătsklinikum Greifs. wald und der Jugendherberge statt.

\section{Universitäts}

\section{Was machen wir?}

Es glbt Workshops und Informationen zus:

- Was macher Hausarzte?

- Wie werde ich Hausaratin-arat?

- Untersuchungskurs

- Alickodiagnostik

- Entspannungsverfahren

- Hilfe, ich werde verklagt!

- Manipulation durch Pharma. werbung?

- Kommunikationstraining

Natürlich werden wir uns auch pernein sam bewegen, Pausen for persornliche Begegnungen und Gespràche haben sowie das schóne Greitswald besichitigen und eine Bootsfahrt machen.

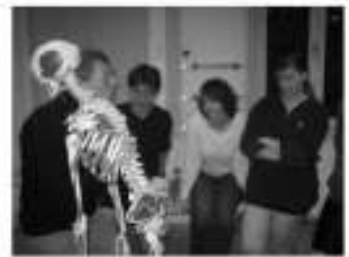

\section{Wer kann teilnehmen?}

Es können sich Medizinstudierende der Wlinischen Semester mit interesse an der Allgemeinmedizin aus ganz Deutschland bewerben.

\section{Entstehen Kosten?}

Be Versand der Teilnahmebestatigung bitten wir um einen Unkostenbeitrag von $60,-\epsilon$ fur Unterkunft, Verpflegung und Verbrauchsmaterial.

\section{Wer sind wir?}

Wir sind eine Gruppe von jungen und atten Allgemeinärztinnen und -ärzten und Arzten /-innen in Weiterbildung, die für iltr Fach „brennen" und diese Begeisterung fur die Allgemeinmedizin gerne an studierende weitergeben wollen. 


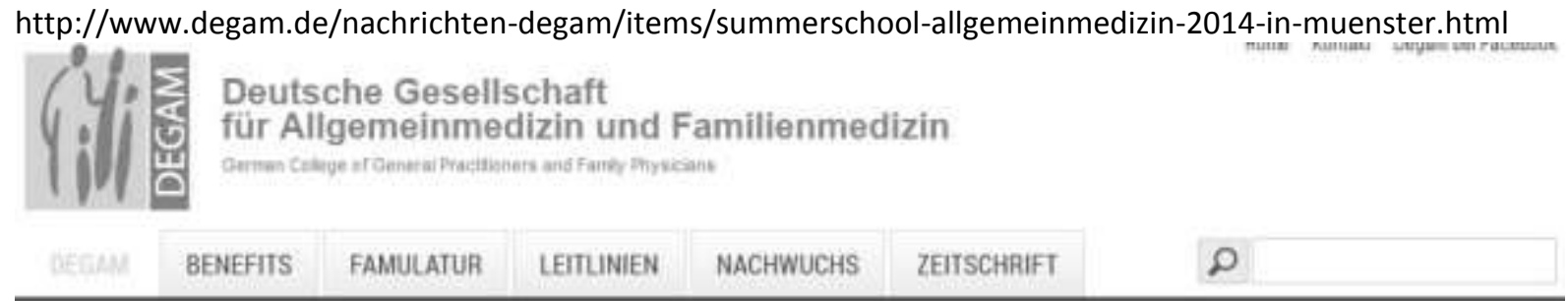

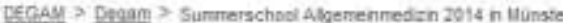

\begin{tabular}{|c|c|c|}
\hline DEGAM & Summerschool Allgemeinmedizin 2014 in & DEGAM MITGLIEDER \\
\hline Uber uns & \multirow{4}{*}{$\begin{array}{l}\text { Münster } \\
\text { Vom 27. . 31. August } 2014 \text { findet in Munster die } 4 \text {. Summerschool fur } \\
\text { Medizinstudierende der kfinischen Semester statt. Die Teilnehmer erarbeiten in } \\
\text { Keingruppen hausarztlich relevarte Themen in der Studienpraxis sorgt der } \\
\text { Einsatz shuatver Lehmethoden für eine große Praxisnahe. Auch das }\end{array}$} & \multirow[t]{2}{*}{ Besutzemane } \\
\hline Presse & & \\
\hline Kongresse \& Veranstaltungen & & \multirow[t]{2}{*}{ Paskwort } \\
\hline Tage der Algemeinmedizin & & \\
\hline Links & Fahmengrogramm verapricht interessant zu werden: Eine der lebenswert nsten & Paswwor veresesent \\
\hline Mitglied werden & Stadte der Weit ladt zu Erhundungen ein & \\
\hline \multirow[t]{2}{*}{ DEGAA intem } & Wetere linformationea sowie die Bewerbungsunterlagen finden Sie hier. & DEGAM NEWS ARCHIV \\
\hline & Zurück & 2014 \\
\hline SEKTIONEN \& AG'S & & 2013 \\
\hline \multicolumn{3}{|l|}{ Forschuing } \\
\hline \multicolumn{3}{|l|}{ Fontbildung } \\
\hline \multicolumn{3}{|l|}{ Quaitatsförderung } \\
\hline \multicolumn{3}{|l|}{ Stuthum und Hochschule } \\
\hline \multicolumn{3}{|l|}{ Versorgungsaufgaben } \\
\hline \multicolumn{3}{|l|}{ Weiterbidung } \\
\hline \multicolumn{3}{|l|}{ AG Diabeles } \\
\hline \multicolumn{3}{|l|}{ AG Famulatur } \\
\hline \multicolumn{3}{|l|}{ AG Komplementarmedixin } \\
\hline \multicolumn{3}{|l|}{ AG Neve Medien } \\
\hline \multicolumn{3}{|l|}{ AG Psychosomatik } \\
\hline AG WIFAMFA: & & \\
\hline
\end{tabular}

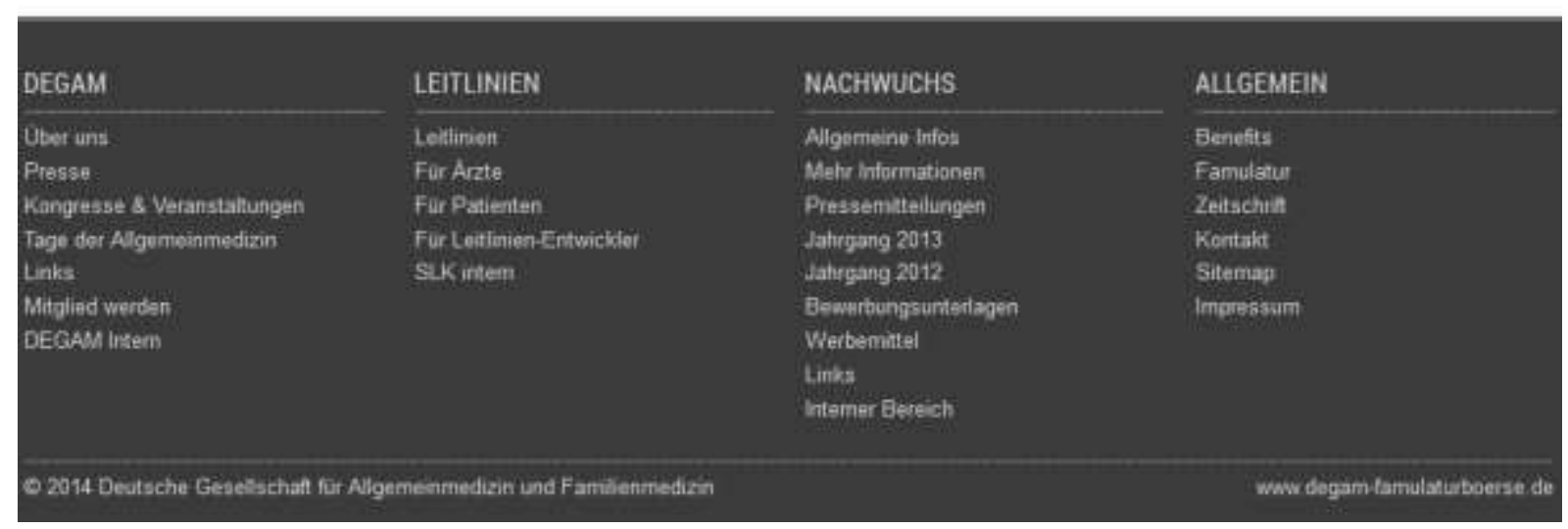


http://www.kvn.de/icc/internet/nav/e1e/e1e70363-b94e-4821-b7d8-f51106fa453d\&sel_uCon=2532c76a1a51-241d-e0d4-515b8ff6bcbb\&uTem=aaaaaaaa-aaaa-aaaa-aaaa-000000000042.htm

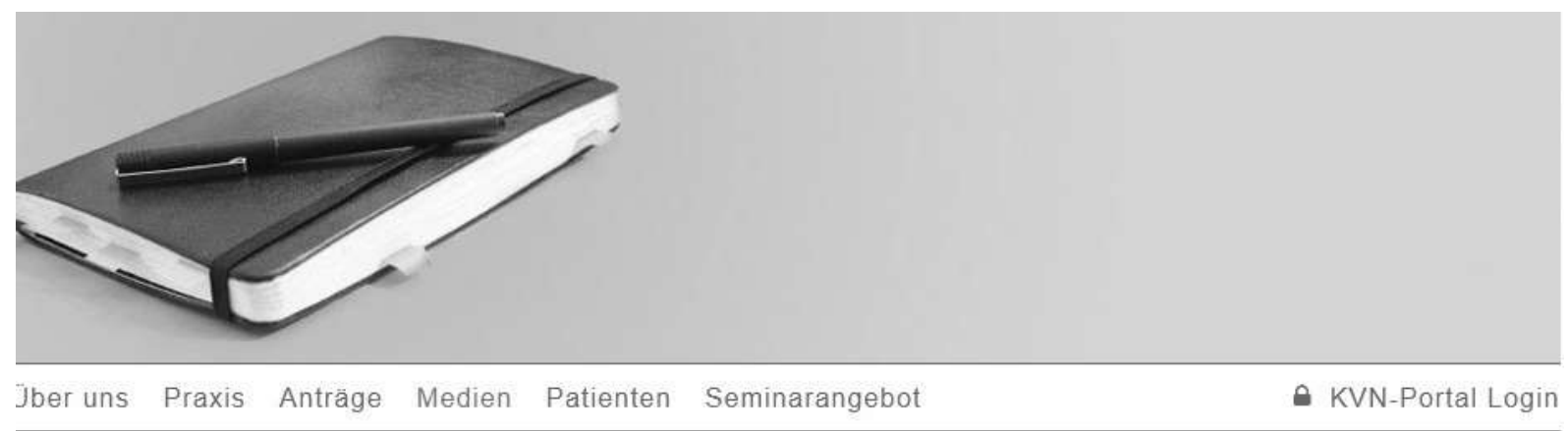

Publikationen
KV on
Im Fokus
Medien-Echo
Ansprechpartne

Ansprechpartner
16.12.2013

\section{Niederlassen in Niedersachsen}

Mit der neuen Dachkampagne „Niederlassen in Niedersachsen“ will die Kassenärztliche Vereinigung Niedersachsen (KVN) im neuen Jahr verstärkt um ärzlichen Nachwuchs werben.

„Mit unserer Kampagne sprechen wir gezjelt jene an, die wir für die ambulante medizinische Versorgung von morgen dringend benötigen: Medizinstudenten, Assistenten in der Weiterbildung und Ärzte, die sich niederlassen wollen ${ }^{2}$, so der Vorstandsvorsitzende der KVN, Mark Barjenbruch heute in Hannover.

Der Altersdurchschnitt der rund 11.000 niedergelassenen Ärztinnen und Ärzte liegt in Niedersachsen bei über 54 Jahren. In den kommenden zwölf Jahren werden in Niedersachsen über 4.200 Ärztinnen und Ärzte in den Ruhestand gehen. Ausreichender Nachwuchs ist im ambulanten Bereich jedoch nicht in Sicht. Besonders betroffen ist die hausärutliche Versorgung. Schon jetzt können sich 390 Hausärzte in Niedersachsen ad hoc niederlassen.

„Uns bricht der Nachwuchs weg", kommentierte Dr. Jörg Berling, stellvertretender Vorstandsvorsitzender der KVN, die prekäre Lage. ,Der Anteil der Absolventen und jungen weitergebildeten Ärzte sinkt Jahr für Jahr, obgleich die Zahl der Zulassungsmöglichkeiten so groß wie nie zuvor ist. Viele dieser jungen Ärztinnen und Ärzte aber wollen nicht mehr 60 oder mehr Stunden in der Woche arbeiten. Sie wollen ein ausgewogenes Verhältnis zwischen Beruf und Familie; , so Berling. Dringend notwendig sei ein gemeinsamer Kraftakt aller Beteiligten im Gesundheitswesen. Ohne ihn drohe eine veritable Versorgungskrise. ,Dann werden in zwölf Jahren in Niedersachsen rund 1.000 Hausärzte fehlen's sagte der KVN-Vize.

Ziel der Kampagne „Niederlassen in Niedersachsen ${ }^{2}$ ist es, dieser möglichen Versorgungskrise entgegenzuwirken und die attraktiven Seiten der haus- und fachärzllichen Praxistätigkeit aufzuzeigen.

Die Kampagne der KVN spricht vor allem Medizinstudenten und Weiterbildungsassistenten als angehende Haus-und Fachärzte an. Ein vielfältiges Informationsangebot rund um das Thema Niederlassung ist auf den neuen Internetseiten unter www.niederlasseninniedersachsen. de zu finden.

\begin{tabular}{l} 
Pressearchiv \\
Artikelsuche von \\
Februar $\checkmark$ \\
\hline $2014 \vee$ \\
bis \\
Februar $\checkmark$ \\
\hline $2014 \vee$ \\
Rubrik \\
alle \\
anzeigen \\
\hline
\end{tabular}




\section{Mehr Landärzte und kürzere Wartezeiten}

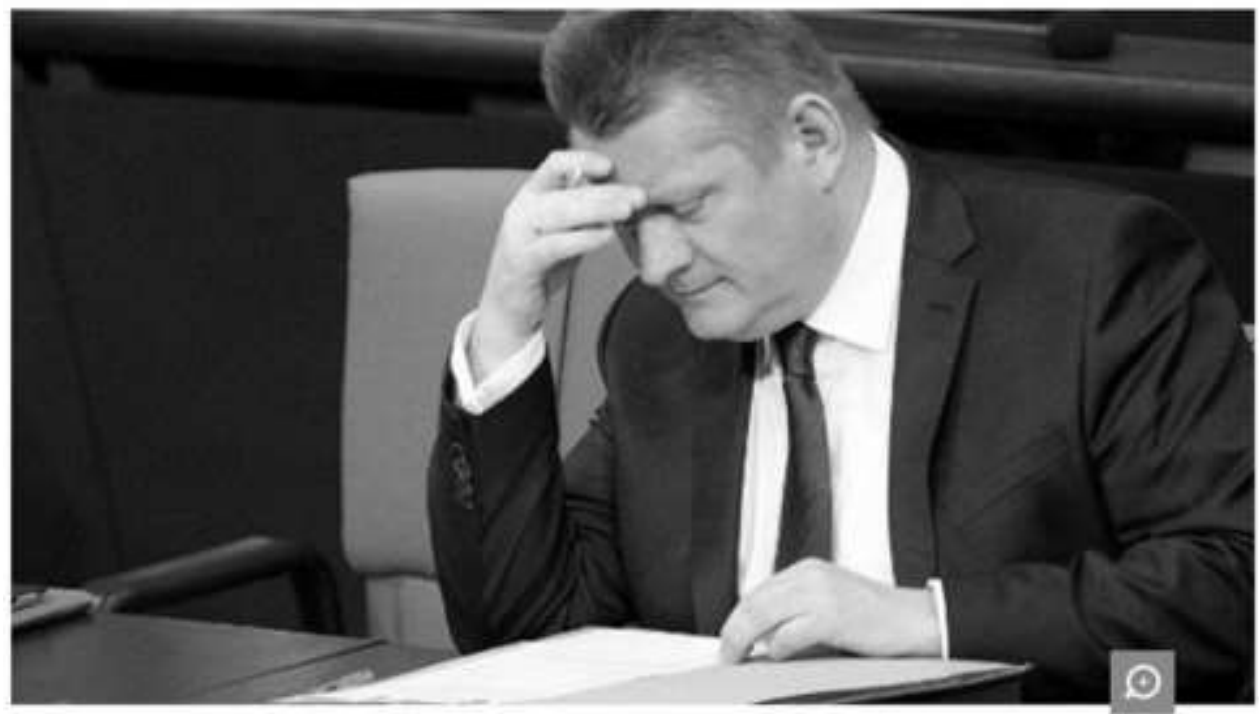

Als neuer Gesundheitsminister muss sich CDU-Politiker Hermann Grohe noch in viele Dinge einlesen

Der neue Gesundheitsminister Hermann Grohe will die arztliche Versorgung in Deutschland deutlich verbessern - auch wenn ihm Erfahrungen fehlen. Kritik daran sieht er gelassen.

Der neue Bundesgesundheitsminister Hermann Gröne (CDU) will die ärztliche Versorgung in Deutschland spürbar verbessern. in einem Interview der "Bild am Sonntag" sicherte der Minister zu, für gesetzich Versicherte die Wartezeiten auf einen Arzttermin zu verküzen. Hintergrund sind anhaltende Kagen, dass diese Versicherten oft sehr viel langer als Privatpatienten aut einen Termin beim Facharzt warten mussen.

Die Koalition habe vereinbart, dass künttig von uberiangen Wartezeiten betroffene Kassenpatienten sich an eine Servicestelle der Kassenarztlichen Vereinigung wenden konnen, sagte Grohe. "Diese vermittelt entweder einen Termin bei einem niedergelassenen Facharzt oder, wenn das nicht geht, eine ambulante Krankenhausbehandlung."

\section{Hohere Arztdichte auf dem Land}

Außerdem möchte sich Gröhe um eine hōhere Arztdichte in landlichen Gebieten kümmern: "ich will mit den Wissenschaftsministern der Lander daruber sprechen, dass junge Leute, die sich verpflichten, eine Pravis im landlichen Raum zu ubernehmen, der Zugang zum Studium erleichtert wird. Dies könnte auch für diejenigen gelten, die ein freiwiliges sozlales Jahr zum Beispiel im Rettungsdienst geieistet haben:

Der Minister kann sich einen Notentbonus als Anreiz vorstellen. "In einigen Bundesłandern und an manchen Hochschulen werden entsprechende Regelungen ja auch schon erfolgreich praktizent"

Grundlegende Kritik an seiner Personalie sieht Grohe indes gelassen entgegen. "Ein ausgewiesener Gesundheitspoiltiker bin ich in der Tat nicht. Aber ich habe in sieben Jahren als Vorsitzender des Diakonischen Werks in meiner Heimatstadt Neuss wichtige Erfahrungen in der Altenplege, der Gemeindepsychiatne und der Hospizarbeit gemacht", sagte er gegenuber "Bild am Sonntag". 


University of MinNEsOTA
Driven to Discover

What's Inside

About RPAP

Faculty and Staff

Outcomes.

Preceptors

Publications

Resources

RPAP Teaching Sites

Rural Observation

Experience

Students

Make a Gift

RPAP Home

Family Medicine and

Community Health

Home > About RPAP

\section{About RPAP}

Who

Medical students who are interested in family medicine, primary care, or rural medicine specialties are strongly encouraged to apply to the Rural Physician Associate Program. Students from both the Twin Cities and Duluth campuses may apply.

\section{What}

RPAP is a unique opportunity in clinical training wherein third-year medical students live and train for nine months in non-metropolitan communities under the supervision of preceptors who are family physicians. These preceptors mentor students in the professional and personal aspects of being a physician.

RPAP students learn clinical medicine, procedures, community health, and the business of medicine. They experience the full spectrum of rural medical care as they follow patients and their families through a disease process or pregnancy.

Students see patients in clinic, hospital, emergency room, nursing homes, hospice, at home, and in the community. Each student is the only student in a community and therefore has a greater opportunity to get hands-on experience in a variety of procedures and specialties.

RPAP was established in 1971 to encourage students to practice in rural areas throughout Minnesota. Over 1,300 students have participated in the program, and two out of three former students practice in Minnesota, two out of three practice in rural locations, and four out of five are in primary care.

\section{Where}

Over 110 Minnesota communities with populations ranging from 350 to 30,000 have been teaching sites. Practices vary from small family medicine clinics to large multi-specialty outpatient centers. Hospitals range in size from 15 to 140 beds. A majority of sites have at least one RPAP alum.

\section{Why}

RPAP gives students a strong foundation in clinical and communication skills and a confident professional approach. Students develop trusting and respectful relationships with physicians, clinic and hospital staff, and patients. The length of the experience allows time for students to become involved in the business of providing health care services as well as in community health education.

The RPAP experience brings to life the American College of Graduate Medical Education's competencies in patient care, medical knowledge, communication skills, professionalism, practice-based learning, and systems-based health care.

RPAP students learn more than just rural medicine. They and their families experience all aspects of rural community life by living in their host towns during the training period. Students become part of the community by joining local sport teams, drama clubs, bands, churches, and civic groups. Many lifelong friendships have been formed through students' involvement in the host communities.

Minnesota's rural lakes and forests offer ample opportunity for RPAP students to pursue fishing, crosscountry skiing, camping, and other outdoor activities during their free time.

\section{Notice of Privacy Practices}

\section{Return to: Family Medicine : Medical School : Academic Health Center : $U$ of $M$ Home}

(c) 2014 Regents of the University of Minnesota. All rights reserved The University of Minnesota is an equal opportunity educator and employer Last modified on May 16, 2012
Twin Cities Campus: Parking \& Transportation Maps \& Directions Directories Contact U of M Privacy 


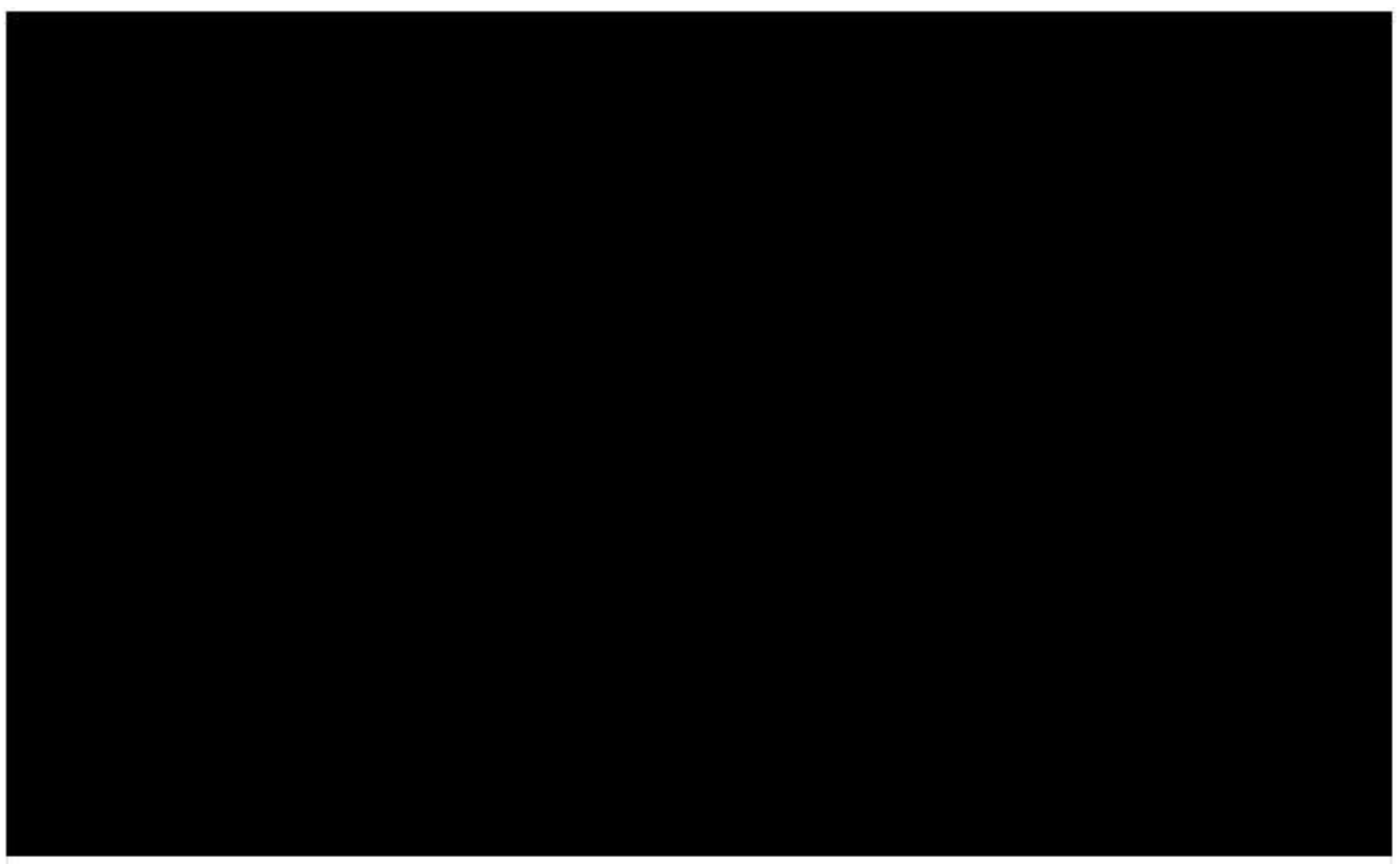

\section{University of Minnesota - Medical School: Duluth Campus}

UMN Health - 287 videos
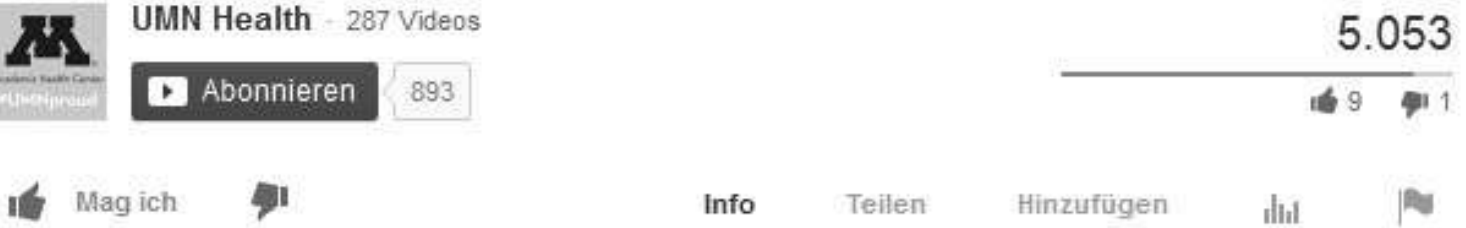

Info

Teilen

Hinzufügen

Whil

Hochgeladen am 09.11.2010

Driven to discover and committed to advancing health. We are one of the country's top medical schools with campuses in

Minneapolis-Saint Paul and Duluth. 


\section{Danksagung}

Für die freundliche Überlassung des Themas der Dissertation, die gewährte Forschungsfreiheit sowie die hilfsbereit unterstützende und konstruktive Betreuung danke ich herzlich Herrn Prof. Dr. disc. pol. Wolfgang Himmel.

Mein besonderer Dank gilt vor allem auch Frau Dr. med. Celia Richter für die intensive Betreuung meiner Dissertation. Durch ihre engagierte, aber besonders auch durch ihre herzliche Art wurde mir das Schreiben der Arbeit maßgeblich erleichtert.

Dr. rer. pol. Christin Löffler danke ich für die Unterstützung auf dem Gebiet der qualitativen Forschung.

Des Weitern bedanke ich mich bei allen Hausärztinnen und Hausärzten, die sich so freundlich an unserer Studie beteiligt haben und die Datengewinnung so unkompliziert gestaltet haben. 